\title{
Bleeding Risk in Interventional Pain Practice: Assessment, MANAgement, AND ReVIeW Of the Literature
}

\author{
P. Prithvi Raj, MD, Rinoo V. Shah, MD, Alan D. Kaye, MD Ph D, Stephen Denaro, BS, and Jason M. Hoover, BS
}

The rarity of published bleeding complications with respect to the practice of interventional pain medicine suggests two possibilities: techniques are being performed in a manner to minimize bleeding or the process of hemostasis is very forgiving. Hence, bleeding complications may increase if techniques are not performed with due skill or if the process of hemostasis is impaired. Interventional pain physicians may be well acquainted with the technical

All interventional pain procedures carry an inherent risk of major and minor bleeding, but this risk is unknown. Literature on this subject, with the exception of neuraxial techniques, is sparse. Several reasons account for this: 1 . Major bleeding episodes are so rare that no study has sufficient statistical power to assess this risk; 2. Reliance on published reports or postmarketing surveillance data may underreport major bleeding episodes; 3 . A selection bias may be present in that patients with an elevated bleeding risk may be offered noninvasive pain management strategies; 4 . The denominator, i.e., number of specific procedures performed, is unknown.

Interventional pain physicians will have to assess bleeding risk based on their own clinical experience and appraisal of available literature. The adoption of guidelines, which apply to neuraxial techniques (1) and other clinical scenarios (2), may or may not be relevant to interventional pain management.

In fact, guidelines published by several European and American anesthesiol-

From Department of Anesthesiology and Pain Services International Pain Institute, Texas Tech University Health Sciences Center, Lubbock, Texas. Address Correspondence:P.Prithvi Raj, MD 4748 Matterhorn Way, Antioch, CA 94531

Email: prithviraj7@comcast.net.

Funding: No financial support was obtained in preparation of this manuscript.

Conflict of Interest: None aspects of procedures, but the degree of their expertise in the field of coagulation is unclear.

This monograph will review coagulation physiology, coagulation pathophysiology, common anticoagulants, and minor and major bleeding complications associated with interventional pain and regional anesthetic procedures.

This manuscript will present a tool to help stratify the risk of bleeding with specific tech-

ogy societies (3-5) only define the risk of significant bleeding, spinal hematomas, for neuraxial procedures in the presence of anticoagulation. The recently updated American Society of Regional Anesthesia guidelines only briefly address the risk of significant bleeding following a few, select non-neuraxial blocks (5). Guidelines for assessing bleeding risk with most interventional pain procedures do not exist. However, the creation of such guidelines is a challenge for several reasons: 1 . interventional pain procedures are heterogeneous, in terms of procedure-related technical complexity and bleeding risk; 2 . the practice of interventional pain management is the purview of a broad range of practitioners, with a wide specialty and skill base, unlike the practice of neuraxial anesthesia; and 3. there are very few reports of bleeding complications with non-neuraxial procedures. Hence, advice about minimizing bleeding risk has to be provided without sufficient information.

Even if guidelines did exist, concerns about malpractice and medico-legal disputes might influence one's clinical judgment. In an analysis of the American Society of Anesthesiology (ASA) Closed Claims database, Kroll et al (6), noted that the mechanism of nerve injury following an anesthetic was rarely discussed or explored with any methodological rigor. Payments, nonetheless, were made. An updated evaluation of the ASA Closed Claims database was conducted in 1999 niques and specific hemostatic abnormalities. The Overall Risk of Significant Bleeding score may help interventional pain practitioners in their individualized assessment of bleeding risk. If used collectively, this tool may help improve patient safety and data collection, with respect to bleeding complications.

Keywords: Bleeding, hemostasis, Interventional Pain Management, regional anesthesia, anticoagulation

(7). The proportion of claims for nerve damage remained constant. However, spinal cord injury secondary to regional anesthetic and interventional pain management techniques became the leading cause of these claims. Payments were often made despite the practice of standard of care (7). This suggests that lay perceptions about causality in nerve injury play a more important role than actual facts. Practitioners are apprehensive about these issues.

The use of percutaneous interventions for the diagnosis and treatment of chronic pain conditions is gaining wider public acceptance. There is a growing body of evidence supporting the utility of these procedures (8). As the population ages, the prevalence of disorders requiring anticoagulant therapy and interventional pain procedures will increase. The risks of bleeding must be weighed against the benefits of the procedure.

Bleeding risks may outweigh the concerns about thromboembolism and practitioners may stop anticoagulation prior to a procedure (9). Ideally, there must be cooperation between the patient, the proceduralist, and the physician managing the anticoagulation. Ultimately, a decision to continue with or abandon a procedure must be made. Guidelines and reviews of relevant literature may help clinicians make appropriate decisions, improve uniformity in patient care, and perhaps, reduce adverse outcomes. 
In this article, we will review coagulation physiology, coagulation pathophysiology, and common anticoagulants. We will survey the literature concerning minor and major bleeding complications that are associated with specific interventional pain management and regional anesthesia procedures. Finally, we will present an approach to assessing the bleeding risk associated with these procedures. Herein lay the greatest difficulties. Bleeding risk assessment is arguably one of the most relevant issues to interventional pain practice, but the most difficult to carry out. Our approach stratifies this risk based on technique-specific and patient-specific factors.

\section{Physiology of Coagulation}

The delicate and complex process of hemostasis involves a series of mechanisms that serve to limit blood loss following vascular damage via clotting, while preserving the fluidity of blood at other times. A significant component of the blood clotting process is the thrombus. However, a thrombus may be formed when blood clotting occurs inappropriately within a major vessel and can lead to vascular occlusion (10). Under normal circumstances, equilibrium between clotting and bleeding is tightly regulated with the assistance of multiple activators, inhibitors, cofactors, and feedback loops, both positive and negative. Pathologies may disrupt that equilibrium, leading to either hemorrhagic or thrombotic complications. Accordingly, hemostasis disorders can be broadly classified into those that lead to abnormal bleeding and those that lead to abnormal clotting (10). Furthermore, these disorders can be characterized by specific abnormalities of platelets, clotting factors, coagulation inhibitors, or thrombolytic/ fibrinolytic processes (11).

Hemostasis involves three linked processes that occur almost simultaneously. Primary hemostasis is the formation of a friable platelet plug. Coagulation or secondary hemostasis is the reinforcement of the friable platelet plug by the formation of a fibrin clot. Fibrinolysis or clot lysis removes thrombi and permits blood flow through recanalized vessels (11).

\section{Primary Hemostasis and Platelets}

Normal platelet actions, such as adhesion and activation, are central events in response to endothelial injury. Primary hemostasis is immediate and results in the formation of a friable platelet plug that temporarily arrests bleeding, until clot formation (coagulation) and repair can occur. Platelets (thrombocytes) are minute, round or oval discs that are one to four micrometers in diameter. They are formed in the bone marrow from megakaryocytes, which are extremely large hematopoietic cells located in the bone marrow; megakaryocytes fragment into minute platelets, within the bone marrow. Alternatively, megakaryocytes can fragment soon after entering the vascular system, especially as they try to squeeze through pulmonary capillaries (10).

Platelets have many of the functional characteristics of whole cells, but they do not have nuclei and they cannot reproduce. Their cytoplasm contains active components: 1 . Contractile proteins that cause platelet contraction, such as actin, myosin, and thrombosthenin; 2. Remnants of the endoplasmic reticulum and the Golgi apparatus, in order to synthesize various enzymes and to store large quantities of calcium ions; 3. mitochondria and enzyme systems that can form adenosine triphosphate and adenosine diphosphate (ADP); 4. Prostaglandin synthesis machinery, which then allows vascular and local tissue reactions; 5 . Fibrinstabilizing factor, and 6. A growth factor that causes vascular endothelial cells, vascular smooth muscle cells, and fibroblasts to multiply, grow in size, and thus, repair vascular wall injury(12).

The cell membranes of the platelets are important. A coat of glycoproteins repulses adherence to normal endothelium, yet permits adherence to injured areas of the vessel wall. In addition the platelet membrane contains large amounts of phospholipids that play several activating roles at multiple points in the blood-clotting process. Thus, the platelet is an active structure. Its half-life in the blood is 8-12 days and its functional processes run out over several weeks.

Despite the important role of platelets, primary hemostasis involves several other components and mechanisms. For example, endothelial cells display surface membrane proteins that resist thrombogenesis. However, when disrupted, a subendothelial layer is exposed: this layer contains a collagen matrix and smooth muscle cells that are highly thrombogenic. Proteins in the subendothelial tissue stimulate platelet adhesion and set the coagulation cascade in motion(12).

\section{Platelet Adhesion}

Platelets, due to their small size and the kinetics of blood flow, i.e., slower blood flow near the perimeter, preferentially circulate and align themselves along the walls of blood vessels (11). When the endothelium is denuded platelets will adhere to the subendothelial collagen layer via von Willebrand factor (vWF). VWF is a protein that is present in plasma and the subendothelial tissue matrix. The platelet glycoprotein $1 \mathrm{~b}$ receptor (GP1b) binds to vWF. This interaction is integral to the process of "platelet adhesion" (11). An abnormality in the vWF:Gp1b receptor-ligand complex may result in bleeding tendencies. For the platelet plug to develop further, platelet activation and aggregation must also occur. Changes in vascular blood flow can result in stasis and turbulence, both of which contribute to platelet aggregation and blood clotting (11).

\section{Platelet Activation}

Adhesion of platelets to subendothelial structures leads to "platelet activation" : 1. Platelet glycoprotein (Gp) fibrinogen receptors (GpIIb/IIIa) are expressed on the luminal side; 2 . Platelets change shape from flattened discs to spheroids with numerous pseudopods; and 3. Platelets undergo a granule release reaction, wherein alpha granules and dense bodies are extruded and compounds, such as ADP, serotonin, clotting factors $\mathrm{V}$ and XIII, and other chemical mediators, are released. Collagen and thrombin are potent platelet activators, whereas ADP, epinephrine, prostaglandin endoperoxides, platelet activating factor $(\mathrm{PAF})$, serotonin, and $\mathrm{TxA}_{2}$ are weaker activators. All of these mediators and processes are vital to primary hemostasis and the subsequent coagulation process (11).

Inactive platelets possess heterogeneous phospholipids and phosphatidyl serine concentrated on the inner leaflet of the cell membrane. Upon platelet activation, phosphatidyl serine flip-flops to the outer leaf and this rearrangement changes the surface charge of the platelet and thus results in procoagulant activity (12). The new procoagulant activity of the platelets is referred to as platelet factor-3 (PF3) activity. Clotting factors interact with the activated platelet surface membrane, form and deposit fibrin, and finally, reinforce the friable platelet plug (11).

Platelet activation is a carefully controlled process that is designed to produce 


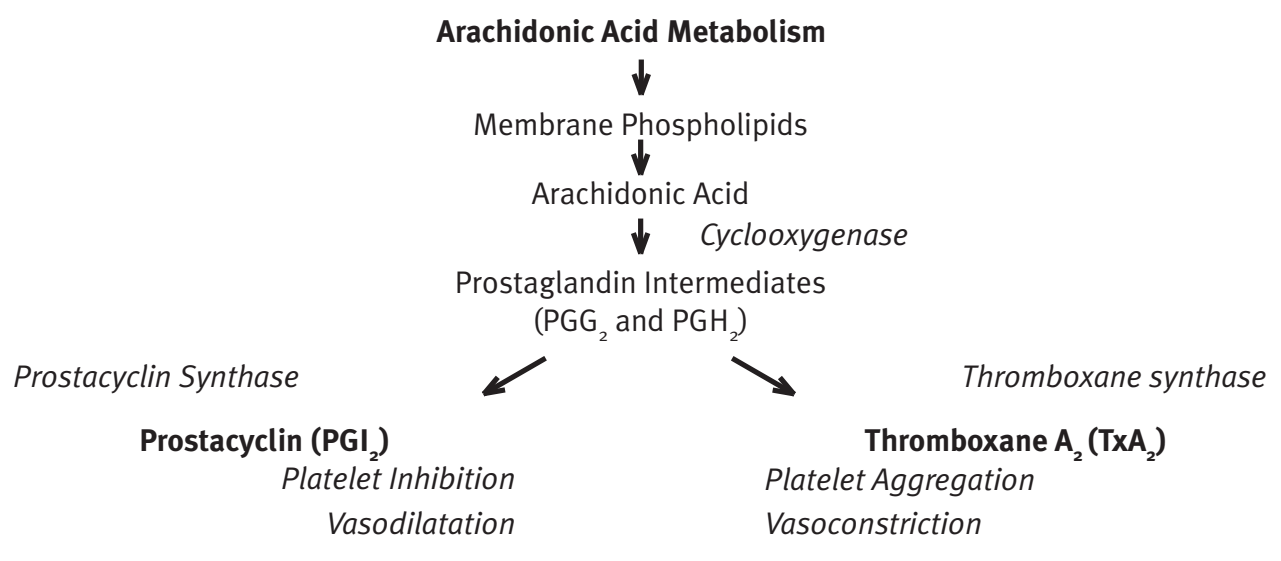

Fig 1. Arachidonic acid metabolism

Adapted from: Petrovitch CT, Drummond JC. Hemotherapy and Hemostasis. In Barash PG, Cullen BF, Stoelting RK (eds), Clinical Anesthesia. 4th Ed. Lippincott Williams \& Wilkins, Philadelphia, PA 2001;213 (11)

an appropriate, but localized response to a damaged area. Activators, such as collagen and thrombin, cause membrane platelet phospholipids to undergo enzymatic changes. Arachidonic acid is converted to thromboxane $\mathrm{A}_{2}\left(\mathrm{TxA}_{2}\right)$ by cyclooxygenase and thromboxane synthase (Fig. 1). TxA, enhances ADP release: ADP is a powerful platelet activator and proaggregant, which actively recruits other platelets to the growing platelet mass/ thrombus (11). Fibrinogen further stabilizes this mass by binding newly exposed platelet GpIIb/IIIa receptors in the presence of $\mathrm{Ca}^{2+}(11)$.

Platelet aggregation can be inhibited by certain factors. Endothelial cells arrest platelet aggregation beyond the area of vascular injury through secretion of prostacyclin $\left(\mathrm{PGI}_{2}\right)$, a potent vasodilator and platelet activation inhibitor. Any imbalance in the production of the two prostaglandins, thromboxane $\left(\mathrm{TxA}_{2}\right)$ or prostacyclin $\left(\mathrm{PGI}_{2}\right)$, can lead to a defect in primary hemostasis or abnormal coagulation.

\section{Coagulation Cascade}

Clotting factors (Table 1) circulate in an inactive form, called a procoagulant molecule or proenzyme. During the process of coagulation, a portion of this protein molecule is cleaved off and the remaining protein becomes an active cleavage enzyme (serine protease). The "activated clotting factor," designated by an "a" after the Roman numeral clotting factor, cleaves off a portion of the next procoagulant clotting factor, which "activates" that factor in succession (Fig. 2). In a continuing cascade, one factor "activates" another, until soluble fibrinogen (factor I) is cleaved to form insoluble fibrin. Fibrinogen is a high-molecular-weight protein (MW-340, 000) that occurs in the plasma in quantities of 100 to $700 \mathrm{mg} / \mathrm{dl}$. Fibrinogen is formed in the liver, and liver disease occasionally decreases the concentration of circulating fibrinogen, as it does the concentration of prothrombin (11).

Table 1. Clotting factors and their synonyms in the blood

\begin{tabular}{|l|l|}
\hline CLOTTING FACTOR & SYNONYMS \\
\hline Fibrinogen & Factor I \\
\hline Prothrombin & Factor II \\
\hline Tissue Factor; Tissue Thromboplastin & Factor III \\
\hline Calcium & Factor IV \\
\hline Proaccelerin; Labile Factor; Ac-globulin (Ac-G) & Factor V \\
\hline $\begin{array}{l}\text { Serum prothrombin conversion accelerator (SPCA); Proconvertin; Stable } \\
\text { Factor }\end{array}$ & Factor VII \\
\hline $\begin{array}{l}\text { Antihemophilic factor (AHF); Antihemophilic Globulin (AHG); Antihe- } \\
\text { mophilic Factor A }\end{array}$ & Factor VIII \\
\hline $\begin{array}{l}\text { Plasma Thromboplastin Component (PTC); Christmas Factor; } \\
\text { Antihemophilic Factor B }\end{array}$ & Factor IX \\
\hline Stuart or Stuart-Prower factor & Factor X \\
\hline Plasma thromboplastin antecedent (PTA); Antihemophilic Factor C & Factor XI \\
\hline Hageman factor & Factor XII \\
\hline Fibrin-stabilizing factor & Factor XIII \\
\hline Pre-Kallikrein & Fletcher Factor \\
\hline High Molecular Weight Kininogen (HMWK) & Fitzgerald Factor \\
\hline Platelets & \\
\hline
\end{tabular}

Adapted and modified from: Guyton AC, Hall JE. In Guyton AC, Hall JE (eds). Textbook of Medical Physiology. $10^{\text {th }}$ Ed. WB Saunders Company. Philadelphia, 2001 (10)
Fibrin is the "mortar" which cements the friable platelet plug. Fibrin first forms a soft gel rather than a solid clot. For structural strength, fibrin requires covalent cross-linking between fibrin monomers. This process is catalyzed by fibrin stabilizing factor (FSF), factor $\mathrm{XIII}_{\mathrm{a}}(11)$.

The last reactions of the clotting cascade (Fig. 2), from factor $X_{a}$ to fibrin, are known as the "final common pathway." The activation of the inactive pro- 
Coagulation Cascade

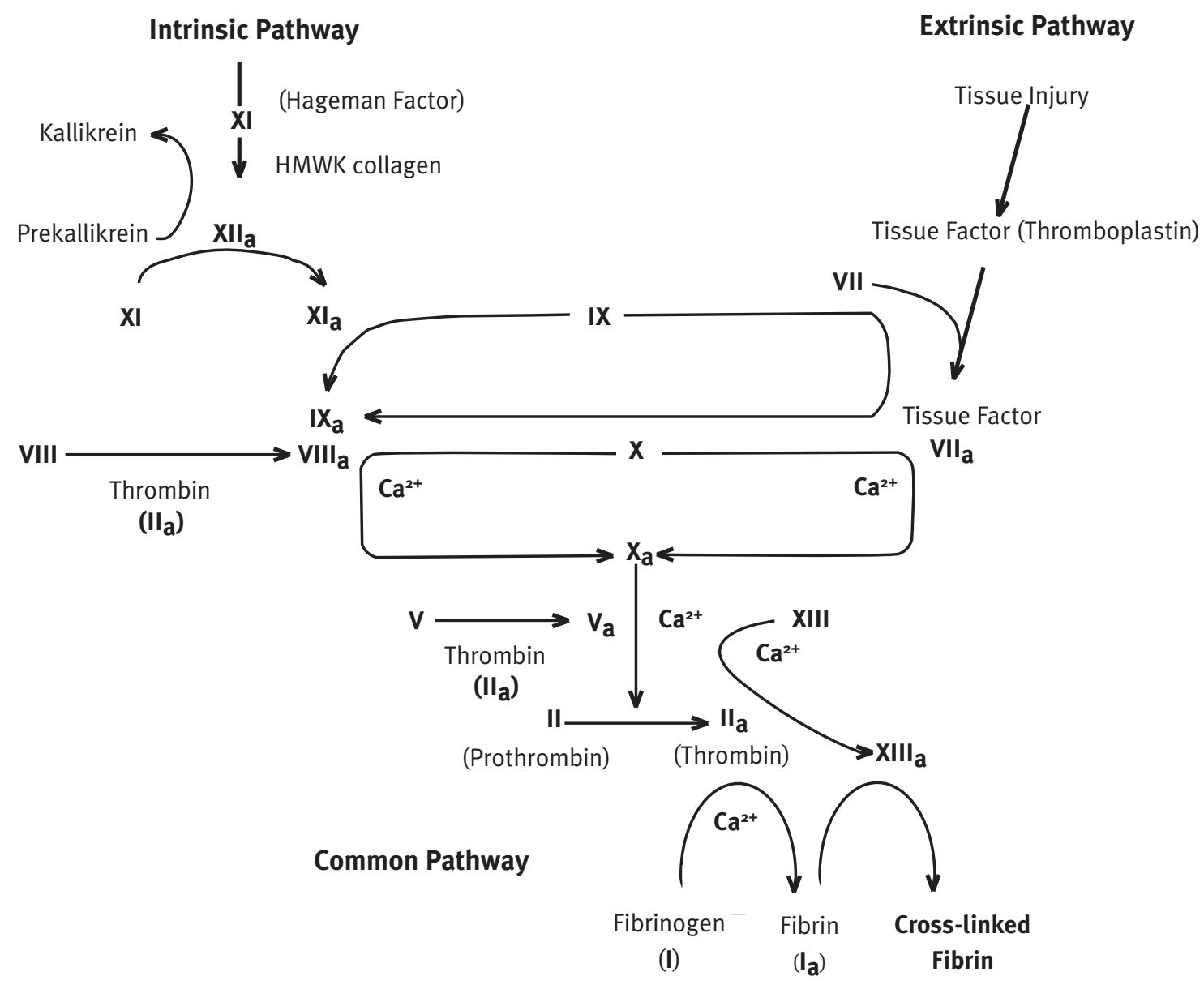

Fig.2 The coagulation cascade.

Adapted from: Mitchell RN, Cotran RS. Hemodynamic Disorders, Thrombosis, and Shock In Kumar V, Collins T (eds). Robbins, Pathology of Disease. $6^{\text {th }}$ Ed, Philadelphia, 1999, 121

coagulant, factor X by a proteolytic cleavage reaction is generated through either the "extrinsic pathway" or the "intrinsic pathway." Once activated, factor $\mathrm{X}_{\mathrm{a}}$ binds with cofactor, factor $\mathrm{V}_{\mathrm{a}}$, and platelet phospholipids (PF3) and the complex converts prothrombin, factor II to thrombin, factor $\mathrm{II}_{\mathrm{a}}$ (Fig. 2).

Prothrombin is a plasma protein, an alpha $_{2}$-globulin, having a molecular weight of 68,700 . It is present in normal plasma in a concentration of about 15 $\mathrm{mg} / \mathrm{dl}$. It is an unstable protein that can split easily into smaller compounds, one of which is thrombin, which has a molecular weight of 33,700 , almost exactly one half that of prothrombin. Prothrombin is formed continually by the liver, and it is continually being used throughout the body for blood clotting. If the liver fails to produce prothrombin, the serum prothrombin concentration will fall to levels too low to provide normal blood coagulation, within $24-48$ hours. Furthermore, vitamin $\mathrm{K}$ is required by the liver for the normal formation of prothrombin and several other clotting factors. Therefore, both vitamin K deficiency and liver disease can reduce prothrombin levels sufficiently, such that a bleeding tendency results.

PF3-bound thrombin cleaves fibrinogen and produces fibrin monomers. Thrombin activates fibrin stabilizing factor (FSF), i.e., factor $\mathrm{XIII}_{\mathrm{a}}$. FSF, in turn, cross links fibrin monomers and increases the structural strength of the fibrin plug (12).
The only protease of the "extrinsic pathway" is factor $\mathrm{VII}_{\mathrm{a}}$, which can be activated by thrombin or factor $\mathrm{X}_{\mathrm{a}}$. However, factor $\mathrm{VII}_{\mathrm{a}}$ only exhibits protease function in the presence of tissue factor (TF), or thromboplastin, a membrane glycoprotein in the subendothelial tissue. Tissue factor and factor $\mathrm{VII}_{\mathrm{a}}$ come in contact only after vascular damage. Hence the name of the pathway since it must require a substance "extrinsic" to blood to proceed (11).

The intrinsic pathway is so named since in-vitro, it can be induced in the absence of any extrinsic tissue components: It requires factors "intrinsic" to blood. This pathway becomes activated only on contact with a negatively charged surface. This surface is provided by constituents of 
the subendothelial tissue following vascular injury. Kallikrein, factor $\mathrm{XII}_{\mathrm{a}}$, and high molecular weight kininogen (HMW$\mathrm{K})$ are the initial contact factors activated by the negatively charged surface and in turn, activate factor XI. Factor $\mathrm{XI}_{\mathrm{a}}$ activates factor IX. Activated factor $\mathrm{IX}_{\mathrm{a}}$ coupled with activated factor $\mathrm{VIII}_{\mathrm{a}}$, binds to the phospholipid surface of the activated platelets (PF3) to form a reaction complex. The $\mathrm{IX}_{\mathrm{a}}-\mathrm{VIII}_{\mathrm{a}}-\mathrm{PF} 3$ reaction complex actives factor $\mathrm{X}$ to produce $\mathrm{X}_{\mathrm{a}}$ and fibrin formation proceeds via a "final common pathway" (12).

Clearly, the intrinsic and extrinsic pathways of the coagulation cascade occur simultaneously, once vascular injury occurs. Tissue factor initiates the extrinsic pathway, whereas the interaction of Factor XII and platelets with collagen in the vascular wall initiates the intrinsic pathway. An especially important difference between the extrinsic and intrinsic pathways is that the extrinsic pathway can be explosive; once initiated, its speed of occurrence is limited only by the amount of tissue factor released from the traumatized tissues and by the quantities of Factors $\mathrm{X}$, VII, and V in the blood. With severe tissue trauma, clotting can occur in as little as 15 seconds. The intrinsic pathway is much slower to proceed, usually requiring one to six minutes to cause clotting (11).

\section{Coagulation Control in the Normal Vascular System}

Coagulation regulation and clot formation must be restricted to the site of damage. Three types of inhibition exist by which extraneous thrombin, factor $\mathrm{II}_{\mathrm{a}}$ is deactivated. The first depends on serine protease inhibitors, serpins, which complex with free clotting factors in the vasculature and block their active sites. Antithrombin III, a member of the serpin family, inhibits numerous clotting factors, particularly factor $X_{a}$ and thrombin. Heparin enhances the activity of antithrombin III and facilitates the formation of inactivated factor $\mathrm{X}_{\mathrm{a}}$ and thrombin complexes (11). Heparin-antithrombin III complexes additionally remove several other activated coagulation factors: $\mathrm{XII}_{\mathrm{a}}, \mathrm{XI}_{\mathrm{a}}$, and $\mathrm{IX}_{\mathrm{a}}$.

The heparin molecule is a highly negatively charged conjugated polysaccharide. By itself, it has little or no anticoagulant property, but when it combines with antithrombin III, the effectiveness of antithrombin III in removing thrombin increases by a hundredfold to a thousandfold and thus it acts as an anticoagulant. Therefore, in the presence of excess heparin, the removal of free thrombin from the circulating blood by antithrombin III is almost instantaneous (11).

Heparin is a powerful anticoagulant, but this property is not clinically apparent due to ordinarily low blood levels under normal physiologic conditions. Parenterally-administered, exogenous heparin will raise serum levels significantly and the anticoagulant properties become readily apparent $(10,11)$.

The second mechanism involves thrombomodulin, an endothelial cell surface protein. Thrombomodulin binds thrombin and this complex activates a serine protease called protein C. Activated protein $C$ couples with serum protein $\mathrm{S}$ on phospholipid membranes and then deactivates clotting factors $\mathrm{V}_{\mathrm{a}}$ and VIII $_{a}$ by proteolysis. Thrombomodulin bound thrombin loses its ability to activate platelets and to convert fibrinogen to fibrin (12).

The third mechanism involves a protein, tissue factor pathway inhibitor (TFPI). TFPI inhibits factor $\mathrm{X}_{\mathrm{a}}$, via a negative feedback loop. Once bound to factor $\mathrm{X}_{\mathrm{a}}$, TFPI will inhibit the tissue factorfactor $\mathrm{VII}_{\mathrm{a}}$ complex and will decrease the proteolytic activation of factor X (11).

\section{Fibrinolysis}

The fibrinolytic system controls the enzymatic degradation of fibrin and is critical for clot removal. Plasminogen, a zymogen, is an inactive protease precursor of plasmin and binds with high affinity to fibrin clots (Fig. 3). Plasmin is a proteolytic enzyme that resembles tryp$\sin$, the most important digestive enzyme secreted by the pancreas. Plasmins digest fibrin fibers as well as other protein procoagulants such as fibrinogen, Factor V, Factor VIII, prothrombin, and Factor XII. Therefore, if plasmin is formed, a hypocoaguable state may occur.

Tissue-type plasminogen activator (tPA) is a serine protease that also binds to fibrin: its activity is minimal in the absence of fibrin. Endothelial cells are the principal physiological source of tPA (12). TPA does not require a proteolytic activation step. When tPA complexes with fibrin, plasminogen will be convert-

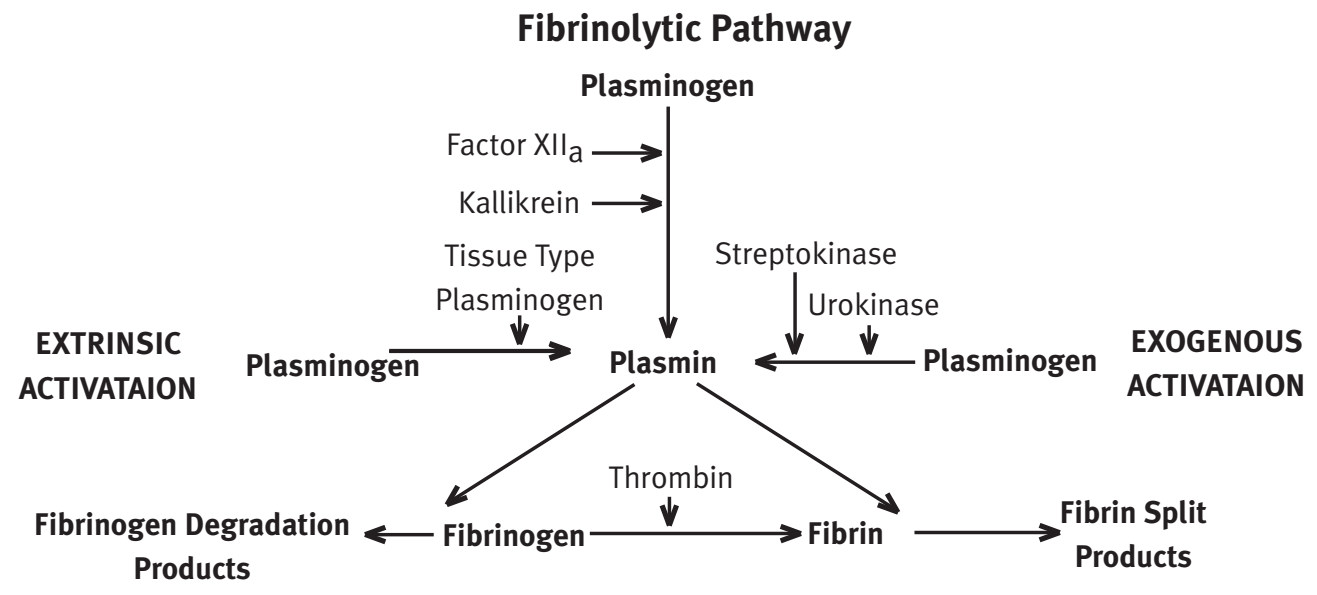

Fig 3. The fibrinolytic pathway

Adapted from: Mitchell RN, Cotran RS. Hemodynamic Disorders, Thrombosis, and Shock: Kumar V, Collins T (eds). Robbins, Pathology of Disease. $6^{\text {th }}$ Ed. Philadelphia, 1999. 
Table 2. Strategies to identify and manage bleeding problems

\section{- Evaluate baseline risk factors}

- Obtain careful history: Significant medical conditions (renal, liver disease), drugs, family history (autosomal dominant, sex-linked), prior bleeding history (inherited vs. acquired), type of bleeding (platelet type vs factor deficiency)

- Physical examination: Petechiae, ecchymoses, mucosal bleeding, hemarthroses, bleeding at venopuncture sites

- Screening tests: CBC, PT, aPTT, thrombin time, routine use of bleeding time not recommended

- Evaluate abnormal/suspicious history, physical examination, or abnormal screening test prior to invasive procedure: May require additional laboratory evaluation (specific factor assays, platelet function tests, von Willebrand analysis, etc.)

- $\quad$ Reduce pre-procedure risk factors

- Prevent pharmacologic coagulopathies

- $\quad$ Allow ample time for elimination of drugs

- Correct abnormal baseline coagulation parameters: Platelet transfusions, fresh or frozen plasma, desmopressin, factor concentrates, dialysis, vitamin K, etc.

- Notify appropriate consultants and blood blank if possible bleeding potential and have plan of action in place

- Enhance post-procedure care: Monitor wound sites

- Follow coagulation tests and factor levels if indicated

- If bleeding occurs: Identify source, is it expected/not expected, is it secondary to local trauma or underlying hematologic derangement

- Administer appropriate replacement products

- $\quad$ Follow with hemoglobin, platelet count, PT, aPTT, fibrinogen, etc.

APTT - activated partial thromboplastin time: CBC - complete blood count: PT - prothrombin time

Adapted from:Cobos E, Cruz J, Day M. Etiology and Management of Coagulation Abnormalities in the Pain Management Patient. Curr Rev Pain 2000 4:413-419 (13)

ed to plasmin, but within the fibrin clot. Hence, enzymatic degradation is confined to where it is needed (11).

Streptokinase, a bacterial protein from streptococci, can also activate plasminogen without proteolytic cleavage: streptokinase binds to the zymogen and induces a conformational change that exposes the active site. Urokinase-like plasminogen (uPA) is a kidney-derived protein present in normal urine and in the plasma. UPA can activate plasminogen to plasmin. UPA and streptokinase, unlike tPA, do not require binding to fibrin for enzymatic activity. They will convert plasminogen to plasmin in the plasma. Streptokinase and urokinase can be used as therapeutic agents for patients with thrombotic diseases (12).

Fibrinogen degradation products (FDPs) and fibrin split products (FSPs) are the end products after fibrin clots are lysed enzymatically. The structures of FDPs and FSPs vary, depending on whether plasmin cleaves fibrinogen, fibrin, or cross-linked fibrin (11). The FDPs are normally removed from the blood by the liver, kidney, and reticuloendothelial system and have a half life of about nine hours; if the rate of production of FDPs exceeds their rate of clearance, FDPs can accumulate. FDPs impair platelet function, inhibit thrombin, and disrupt the cross-linking of fibrin strands. FDPs, themselves, inhibit both primary hemostasis and coagulation (11). An elevation in FDPs indicates a significant bleeding risk: FDP levels serve as a marker of ongoing fibrinolytic activity and the degree of anticoagulation. 


\section{Clinical Assessment of Bleeding Risk}

Understanding the physiology of bleeding is important, but identifying patients at risk for bleeding is clinically relevant. Chronic pain patients represent an extremely diverse group of patients from the standpoint of co-morbid illness. Many may have an underlying clinical bleeding disorder that has not been diagnosed or for that matter, never recognized. Prior to performing any invasive procedure, the practitioner must identify those patients at a specific risk for bleeding. The clinical history, physical exam, and preliminary screening tests should be performed by practitioners, before referring suspected patients to a hematologist.

Patients are at risk for bleeding if they 1 . Present with a current or prior history of bleeding (prior surgical bleeds, recurrent or prolonged epistaxis, hematuria, gastrointestinal bleeding, bleeding after circumcision); 2. Report easy and excessive bruising or bleeding, either without antecedent trauma or with minor trauma, such as injections, small cuts, or tooth extractions; 3 . Have never had prior bleeding problems, but present with a new abnormal bleeding episode; 4 . Have an underlying medical condition, such as renal failure, liver failure, malignancy, or systemic lupus erythematosus, which poses an increased bleeding risk or increased likelihood of abnormal coagulation studies; 5. Have an unreliable, questionable, or impressive family history of bleeding and are going to undergo an invasive procedure; and 6 . Are women that have a history of excessive menstrual blood flow or bleeding with parturition $(13,14)$.

During the history taking process, practitioners should ask about the type of bleeding. Patients with coagulation abnormalities of secondary hemostasis, such as hemophilia, usually manifest deep muscle hematomas or hemarthroses. Patients with platelet abnormalities such as von Willebrand disease typically experience mucocutaneous, i.e., "superficial", bleeding such as epistaxis, gingival bleeding, bruising, petechiae, gastrointestinal bleeding, such as melena, hematuria, or excessive menstrual flow (Table 2). Details of transfusions needed for bleeding episodes should be elicited. The physical exam can help identify the type of bleeding and bleeding sequelae as well. For instance, many patients with hemophilia may present with a painful swollen joint or develop a hemophilic arthropathy. Patients with platelet disorders may have areas of excessive bruising or petechiae.

Detailed past medical, medication, and family histories are important, particularly when trying to distinguish between hereditary and acquired bleeding disorders.

Patients should be queried about the consumption of well recognized anticoagulants, such as coumadin, clopidogrel, aspirin, and low molecular weight heparins. The practitioner may ask about anticoagulant use in several different ways to ensure that the patient understands what is being asked. Patients should be asked about their consumption of over the counter products and herbal medicines. Numerous cold and cough remedies may contain aspirin or non-steroidal anti-inflammatory drugs. Likewise the drug history is important, since even drugs not typically associated with bleeding may cause bleeding: common antidepressants that inhibit serotonin reuptake have been associated with increased blood loss during certain types of surgery (15).

Patients with severe hereditary bleeding abnormalities typically present at infancy and have a life-long bleeding history. Patients with mild but hereditary disorders may present as adults and have little or no abnormal bleeding history.

Hereditary disorders will frequently affect other family members of the patient. Directed family history questions should concentrate, for instance, on bleeding episodes in male relatives over several generations. The latter is important, since inherited diseases, such as hemophilia, might skip a generation if that generation had female carriers. Disorders that are transmitted in an autosomal dominant fashion, such as von Willebrand's disease, will have a positive family history. Some patients may lack an impressive family history and still have an inherited bleeding disorder, if they suffer from a rare autosomal recessive condition. Also, some typically sex-linked inherited bleeding disorders, such as hemophilia A or B, may occur due to spontaneous mutations (13).

Routine laboratory tests of blood urea nitrogen, serum creatinine, serum electrolytes, liver function, and complete blood count may be necessary in order to identify an underlying renal, hepatic, hematological, infectious, or oncologi- cal disease. These illnesses may increase bleeding risk. Severe systemic disorders, such as sepsis or malignancy, may lead to major bleeding problems (13).

In the absence of a bleeding history the need for routine laboratory tests may not be necessary (16). The best preoperative screening test to predict bleeding continues to be a carefully conducted clinical history that includes family, dental, obstetric, surgical, traumatic injury, transfusion, and drug histories (16). Once patients have been identified to be at increased risk for bleeding, then tests of clotting function may be useful. Another caveat is that specific coagulation assays rarely correlate with blood loss, but they do provide specific diagnoses, which can guide treatment (17).

\section{Tests of Clotting Function}

\section{Platelet Count}

The normal concentration of platelets in the blood is between 140,000 and 445,000 per microliter (13). The platelet count is an extremely important routine investigation, as platelet deficiency (thrombocytopenia) will increase bleeding tendency. Thrombocytopenia is defined as a platelet count $<150,000$ per microliter. A platelet count performed by automated cell counters may erroneously read a low platelet count due to pseudothrombocytopenia. Pseudothrombocytopenia occurs due to the formation of platelet clumps: platelet clumping occasionally occurs in blood samples that are drawn in ethylenediaminetetracetic (EDTA) anticoagulant tubes. In order to confirm the diagnosis of pseudothrombocytopenia, a peripheral blood smear should be examined and a repeat blood sample should be redrawn in tubes containing a different anticoagulant, such as a citrate of heparin (13).

\section{Bleeding Time}

The bleeding time is an indicator of primary hemostasis in vivo $(18,19)$. Bleeding time prolongation occurs in patients with quantitative and qualitative platelet disorders (16). Specifically, the bleeding time assesses platelet plug formation at a skin wound: a small skin incision is made and the time required for blood flow to cease is the bleeding time (16). 
The Ivy bleeding time involves making a skin incision along the volar forearm and using a blood pressure cuff to prevent vessel collapse (16). The Ivy bleeding time is a common pre-operative test to assess the status of anti-platelet therapy $(20,21)$. If the bleeding time is $<10$ minutes, some practitioners consider central neuraxial blocks to be safe $(20,22)$. Others assert a cut-off of 6 minutes (21).

Several modifications of the bleeding time have been introduced, such as the template technique to standardize the length and depth of incision and the use of disposable devices $(19,23)$. Disposable devices are simple to use, incur minimal physical trauma, and are essentially painless (23). In using a disposable device, Babson et al (23), reported a mean bleeding time of $4.1 \mathrm{~min}$ with a $95 \%$ range of 2.2-7.0 min in 47 normal adults. The standard deviation of duplicate bleeding times and day-to-day variation was 0.7 and 0.9 min, respectively. In a double-blind crossover study of 20 normal adults, the mean bleeding time increased from 3.7 to 6.2 min after ingestion of $1000 \mathrm{mg}$ of aspi$\operatorname{rin}(23)$.

Nonetheless, the utility of the bleeding time has been questioned (16). The bleeding time at the skin lacks sensitivity and specificity in predicting hemorrhage, following an invasive procedure (24-26). The bleeding time cannot predict the degree of intra-operative hemorrhage or bleeding at gastric biopsy sites, in patients receiving aspirin therapy (2428). There is no correlation between hip surgery-associated blood loss and bleeding time prolongation, in the presence of aspirin. This held true irrespective of whether bleeding time prolongation was defined as $>10$ minutes or $>4$ minutes above baseline (27).

Bleeding times cannot be used reliably to identify patients who may have recently consumed antiplatelet drugs (16). In vitro platelet aggregation tests may take up to 7 days to normalize, but the bleeding time may normalize in 3 days (29). This implies that platelet function can still be abnormal, even if the bleeding time is normal shortly after stopping aspirin (21).

The bleeding time may frequently be prolonged due to improper performance of the test. Only experienced laboratory personnel should perform a bleeding time (16). The bleeding time is not recommended as a routine test $(16,24-26)$, despite current widespread use. Bleeding times should be limited to those patients with a normal platelet count and a history of bleeding that suggests platelet dysfunction.

\section{Platelet Function Analysis}

Platelet function analyzers were recently introduced to study primary hemostatic disorders. Specifically they offered several advantages over standard platelet function studies, such as improved sensitivity and specificity and ease of use. They are used 1. As a screening tool for von Willebrand disorder and various platelet disorders; 2. For monitoring the response to desmopressin acetate (DDAVP) therapy in both VWD and platelet disorders; 3 . For monitoring response to antiplatelet drug therapy, such as aspirin; and 4. For evaluating primary hemostasis in various clinical disorders or during surgical procedures (30).

One method of platelet function analysis, the PFA-100, utilizes whole blood. Whole citrated blood is passed through a capillary tube into the central aperture of a membrane. This mimics in vivo shear stress conditions (30). The membrane is coated with collagen or a platelet agonist, such as epinephrine or adenosine diphosphate. A platelet plug forms over the aperture. The time required to close the aperture and stop capillary blood flow is defined as the Closure Time (30-32). Normal reference ranges for Closure Time with collagen/adenosine diphosphate (C/ADP) is $77-133$ seconds and with collagen/epinephrine (C/epi) is 98-185 seconds $(30,33)$.

The PFA-100 is not sensitive in detecting fibrinogen defects, heparin effects, and hemophilia A or B. The PFA-100 closure time may be prolonged in the setting of thrombocytopenia or antiplatelet drug therapy. The PFA-100 has been used to monitor therapy with glycoprotein IIB/IIIa antagonists, non-steroidal anti-inflammatory drugs and ticlopidine. The PFA-100 may be more sensitive than a classical bleeding time and closure times may exhibit a dose dependent effect (30, 34). Additionally, anti-platelet drugs may exhibit differential effects on closure time, depending on whether a collagen/ADP or collagen/epinephrine cartridge is used (30). In fact the laboratory at our institution considers a prolongation of closure time in the presence of C/epi, but not in the presence of $\mathrm{C} / \mathrm{ADP}$ to be suggestive of an aspirin-like effect.

Overall the PFA-100 is simple to use and has a high sensitivity to particular hemostatic disturbances such as vWD, platelet disorders, and platelet 'affecting' medications. However, the PFA-100 cannot distinguish among these various disorders. Further testing is required if the underlying cause of an abnormal PFA-100 result is unknown (30). Whether the PFA-100 is predictive of significant bleeding following invasive procedures is unknown. One recent study demonstrated that the PFA100 was not able to separate those patients at low risk for bleeding after cardiopulmonary bypass from those who had substantial bleeding (35), even though platelet dysfunction is the most important reason for bleeding after cardiopulmonary bypass (35). Similarly, another study demonstrated a low positive predictive value of abnormal PFAs in detecting postoperative bleeding (36). However, normal PFA values argued against a coagulopathy as the etiology of post-operative bleeding following cardiac surgery: a normal PFA in the presence of significant post-operative bleeding suggests that a surgical cause should be quickly identified and managed (36). Nonetheless, routine use of the PFA100 after cardiac surgery is not useful in predicting post-operative bleeding (37). Others suggest that the PFA-100 may be a useful tool in identifying patients with an elevated bleeding risk prior to performing an epidural procedure (38).

\section{Thromboelastography}

Thromboelastography (TEG) is used to monitor whole-blood coagulation. Specifically, TEG monitors the shear elastic modulus during clot formation in whole or recalcificed citrated blood(17). TEG yields qualitative information about platelet function, thromboplastin generation, and their respective interactions with the intrinsic coagulation cascade to form a stable clot (39). TEG also provides information about fibrinogen and Factor XIII levels, as well (39). TEG is very non-specific (17), but may be more sensitive than traditional coagulation tests in predicting and managing coagulopathies (39). Some limitations of TEG include its 1 . Inability to diagnose a specific hemostatic abnormality; 2. Weak correlation with specific coagulation assays; and 3. Inability to consistently detect the benefits of fractionated blood product therapy(17). TEG may be able to 
monitor fibrinolysis and hemostasis during liver transplantation and consequently, reduce blood transfusion requirements (40). TEG may be useful in predicting the bleeding risk in patients that are going to undergo regional anesthesia (39). The TEG maximum amplitude may be associated with excessive hemorrhage after cardiopulmonary bypass surgery, but no direct correlation with degree of blood loss has been demonstrated $(17,36)$. In fact, TEG, like the PFA-100 has a high negative predictive value in the early identification of surgical bleeding versus a significant coagulopathy (36). TEG has been useful in obstetric anesthesia. The severity of preeclampsia is associated with progressive impairments in hemostasis and this can be assessed with TEG $(17,41)$. However, the ability of TEG to identify which parturients with moderate-severe hypertension can safely receive epidurals is still not known (17). TEG and injury severity were both predictive of early transfusion requirements in major blunt trauma (42). Overall, TEG may improve clinical decision-making in patients undergoing surgery (17). However, there are several problems to universal adoption of TEG as a pre-procedure screening tool. TEG is non-specific. There is a lack of quality assurance methodologies. Finally, studies on the utility of TEG lack sufficient scientific rigor or power (17).

\section{Activated Partial Thromboplastin Time} and Prothrombin Time

Activated partial thromboplastin time (aPTT) and prothrombin time (PT) are measures of the intrinsic (contact phase) and extrinsic pathways, respectively, of the coagulation cascade. Both pathways result in the generation of factor Xa. The reaction then proceeds along a common pathway, resulting in the generation of thrombin, which converts fibrinogen to insoluble fibrin clot. The aPTT and PTT cannot measure factor XIII.

The aPTT mimics intrinsic pathway activation (43). Citrated plasma from the patient is mixed with phospholipid and a surface activator (celite or kaolin), and the reaction is started by the addition of calcium. The contact activation phase of coagulation begins with the generation of active Hageman's factor (factor $\mathrm{Xll}_{\mathrm{a}}$ ), which in turn converts factor $\mathrm{Xl}$ to its active form, and this enzyme together with the cofactor (factor VIII) is responsible for the generation of factor $\mathrm{X}_{\mathrm{a}}(43)$.
A prolonged aPTT will be reported with deficiencies in factor VIII and factor IX, secondary to hemophilia A, hemophilia B, or vWD. Acquired deficiencies can occur with liver disease, which reduces production of vitamin $\mathrm{K}$ dependent factors IX or X, or if a patient is on oral anticoagulant therapy (43). Suchmanet al (44) found that the aPTT was not able to predict the occurrence or absence of hemorrhagic complications in patients at low risk for bleeding. However, the aPTT was useful in predicting post-procedure hemorrhage in patients at higher risk for bleeding, such as liver disease, malabsorption, malnutrition, and acquired coagulopathies (44). The use of aPTT as a screening test should be limited to patients at higher risk for bleeding, following a procedure (44).

Correspondingly, the PT reveals disorders in the extrinsic pathway. The PT is a simple test based on incubation of patient plasma, calcium, and tissue thromboplastin (tissue factor). The addition of tissue factor accelerates the normal clotting time for plasma from five minutes to about ten seconds. In vivo, the extrinsic pathway for factor $\mathrm{X}_{\mathrm{a}}$ generation requires the release of tissue factor VII in the plasma to form an enzymatic complex that cleaves factor $\mathrm{X}$ to factor $\mathrm{X}_{\mathrm{a}}$. Blood removed from the patient is immediately citrated so that none of the prothrombin can change into thrombin. Later, a large excess of calcium ion and tissue factor is suddenly mixed with the citrated blood. The calcium nullifies the effect of the citrate, and the tissue factor activates the prothrombin-to-thrombin reaction by means of the extrinsic clotting pathway.

\section{Table 3. Clotting disorders}

\section{Hereditary Disorders}

von Willebrand's disease

Hemophilia A

Hemophilia B - Christmas disease

\section{Acquired Disorders}

Acquired Hemophilia

Vitamin $\mathrm{K}$ deficiency (malnutrition, biliary obstruction, malabsorption, sterile gut) Liver Disease (complex coagulopathy)

Renal Disease

DIC (uncontrolled bleeding with uncontrolled coagulation)

Drugs-induced Hemorrhage (aspirin, heparin, coumadin)

Immune Thrombocytopenia Purpura (ITP)

Adapted from: Koh M, Hunt B. The management of perioperative bleeding. Blood Reviews 2003; 17: $179-185$ time required for coagulation to take place is known as the prothrombin time because the shortness of the time is determined mainly by prothrombin concentration. The normal prothrombin time is about 12 seconds. In each laboratory, a curve relating prothrombin concentration to prothrombin time is drawn for the method used, so that the prothrombin in the blood can be quantified. However, variations between laboratories in PT values have led to the development of the international normalized ratio, INR. The INR simplistically converts the ratio, $\mathrm{PT}_{\text {patient }} / \mathrm{PT}_{\text {mean normal }}$, to the value expected if the World Health Organization reference thromboplastin were used (43, $45,46)$.

\section{Test for Other Clotting Factors}

Additional tests to assess specific bleeding disorders should be carried out in consultation with a hematologist. Mixing studies, between the patient's plasma and normal plasma may help narrow the differential diagnosis. Specifically, if mixing studies in patients with elevations in PT or PTT normalize, then a factor may be deficient (43). Tests similar to that for PT time have been devised to determine the quantities of other blood clotting factors. In each of these tests, excess of calcium ions and all factors apart from the one being tested are added to oxalated blood at once. Then the time of coagulation is determined in the same manner as for the usual prothrombin time. If the factor is deficient the coagulation time is prolonged. The time itself can then be used to quantitate the concentration of the factor being studied (43). 


\section{Pathophysiology of Coagulation}

Coagulation factors and processes are complex and inter-dependent. Under pathological circumstances, disequilibrium between clotting and bleeding can develop. Defects in coagulation physiology manifest as hemorrhagic or thrombotic disorders. Hemorrhagic disorders can be broadly characterized, based on whether there are problems related to platelets (primary hemostasis), clotting factors (secondary hemostasis), the presence or absence of inhibitors, such as fibrin degradation products (fibrinolysis), or a combination of these three. Furthermore, hemorrhagic disorders may be either hereditary or acquired (Table 3 ).

\section{Hereditary Disorders of Hemostasis}

Hereditary (congenital) coagulation disorders are usually due to the absence or reduced presence of a single procoagulant (47). The three most common hereditary coagulation disorders are von Willebrand's disease (deficiency of von Willebrand factor), Hemophilia A (factor VIII deficiency or classic hemophilia), and hemophilia B (factor IX deficiency or Christmas disease). An understanding of the deficient or absent procoagulant, its elimination half-life following exogenous administration, and the products available for treatment of the coagulation disorder are important in the clinical management of these patients. A hematologist should be closely involved in the management of patients with hereditary or acquired coagulopathies that will be receiving a percutaneous or surgical intervention.

\section{Von Willebrand Disease}

Von Willebrand Disease is the most frequent of inherited bleeding disorders afflicting approximately $1-3 \%$ of the general population (48-50). This coagulation disorder is caused by quantitative or qualitative defects of von Willebrand factor. This results in low factor VIII levels and abnormal platelet adhesiveness, since vWF is necessary for platelet adhesivity to exposed endothelium (51). Von Willebrand Disease is inherited as an autosomal dominant trait, which affects both sexes. There are three types. In type I, there is a reduction in the amount of vWF (52). In type II, vWF levels are normal, but the vWF is functionally abnormal. In type III, there is a severe deficiency in
vWF. The severest form is homozygous but fortunately, very rare (50).

Patients describe a lifelong history of bruising and mild, mucosal bleeding, unlike hemophiliacs $(48,49)$. Prolonged epistaxis or menorrhagia may be present (52). Patients are frequently unaware that they have a bleeding disorder until they undergo surgery or experience trauma (53). Excessive bleeding from surgery or trauma is typically localized to the site of the injury and distant site bleeding, such as that into joints and soft tissues, is uncommon.

Von Willebrand's disease is diagnosed by patient history and laboratory studies: abnormal platelet function assays, bleeding time prolongation, and normal platelet counts. Recently, the PFA-100 was found to have a sensitivity of $96-100 \%$ and a specificity of $95 \%$, when used to screen various subtypes of von Willebrand disease $(31,32)$. Two specific laboratory investigations, vWF:Ag level and Ricof level, are used to evaluate the quantity and functional capacity of von Willebrand factor, respectively (52). The vWF:Ag level can be measured directly and compared to reference values. Amounts $>50 \%$ are considered normal. Ristocetin is an antibiotic that interacts with vWF and platelets to induce aggregation. Platelet aggregation by ristocetin follows a dose-response curve that depends on the amount of ristocetin cofactor, the functional unit of von Willebrand factor. The Ricof level refers to the amount of Ristocetin cofactor. Normal Ricof levels are $>50 \%$, but levels as low as $30 \%$ are not thought to increase bleeding risk. Ricof levels below $10 \%$ are associated with significant bleeding. It is unclear what Ricof level is safe for regional anesthesia (52).

Prophylaxis and treatment options for vWD include desmopressin and factor replacement. Desmopressin raises endogenous factor VIII and von Willebrand factor. The majority of patients with vWD respond to this treatment. Approximately $20 \%$ of patients do not respond to desmopressin and require plasma concentrates to boost factor VIII and vWF levels $(49,50)$. The PFA-100 may be used to monitor response to desmopressin, but not factor replacement therapy $(31,32)$. Estrogen therapy may also improve the bleeding abnormalities of von Willebrand disease (54).

\section{Hemophilia: A and B}

Hemophilia A is a bleeding disorder resulting from a defect or deficiency of coagulation Factor VIII:C . Factor VIII:C is a protein that serves as a cofactor for the activation of factor $\mathrm{X}$ in the coagulation cascade; defects in factor VIII: $\mathrm{C}$ results in a hemorrhagic tendency. $\mathrm{X}$ linked transmission of a recessive genetic trait limits this disease, predominantly, to males and homozygous females. Rarely, heterozygous females may present with severe hemorrhage, presumably caused by "unfavorable lyonization" (inactivation of the normal X chromosome in most of the cells) (53). In males, the incidence is 1:10,000-1:25,000 $(53,55)$. All patients with hemophilia A have normal plasma concentrations of vWF.

In its most severe form, hemophilia $\mathrm{A}$ is a life-threatening and crippling disease. Severely afflicted patients are at risk for spontaneous bleeding into joints, into soft tissues, into neural compartments with subsequent neural compression, into the urinary tract, and into intracranial structures $(53,55)$. Other sites of bleeding include mucosal and cutaneous tissues (55). Spontaneous bleeding in synovial joints results in a progressive hemophilic arthropathy. Central nervous system bleeding is the major cause of death in patients with hemophilia $\mathrm{A}(53,56)$ and is associated with a $30 \%$ mortality rate (56). The incidence of bleeding into the central nervous system is $2.2-7.8 \%$ (56). However, the most common neurological injury in hemophilia is bleeding into peripheral nerve compartments (56). The incidence of peripheral nerve injury ranges from $1.3-20.4 \%$ (56).

The activated partial thromboplastin time $(\mathrm{aPTT})$ is prolonged in these patients, but the platelet count, bleeding time, and prothrombin time (PT) are usually normal. Hence, Hemophilia A is an abnormality of the "intrinsic pathway" of the coagulation cascade (53). Factor VIII assays can confirm the diagnosis of hemophilia (55). There is a direct relationship between the plasma concentration of factor VIII and the severity of bleeding. Normal plasma activity ranges between $0.5 \mathrm{U} / \mathrm{ml}(50 \%)$ to $1.5 \mathrm{U} / \mathrm{ml}(150 \%)$. Severely affected patients have levels below $1 \%$. Moderately affected patients have levels between $2-5 \%$. Mildly afflicted patients have amounts ranging from $6-30 \%$ $(53,57)$. Nonetheless, mildly affected patients can bleed significantly after trauma 
or surgery (57).

Perioperative management is a challenge since major hemorrhaging can be fatal. Factor replacement therapy is necessary to keep levels higher than $30 \%$ ( $0.3 \mathrm{U} /$ $\mathrm{ml})$. Pre-operative Factor VIII levels may have to approach $100 \%(1 \mathrm{U} / \mathrm{ml})$ in order to protect against intra-operative reduction in factor levels secondary to hemodilution. Replacement of Factor VIII above $30 \%$ for 10 days is thought to be sufficient. Factor replacement can be plasma-derived or recombinant. The safety of plasma-derived factors depends on the viral load and degree of viral inactivation in these viruses. Recombinant factor VIII, as compared to pooled blood factor VIII concentrates, theoretically can reduce the risk of viral infections to nil $(55,57)$. Recombinant Factor VII $_{\mathfrak{a}}$, a 'universal' hemostatic agent, maybe used as well to control surgical or trauma-associated bleeding in hemophilia (58).

Hemophilia B (Christmas disease), is an X-linked genetic disorder due to a defective or deficient factor IX molecule resulting in hemorrhagic tendency. The inheritance pattern and the clinical features are indistinguishable from those of hemophilia A. Diagnosis of hemophilia $B$ depends on the presence of low or absent plasma factor IX concentrations in the presence of normal factor VIII activity. As with hemophilia A, the PTT will be prolonged and PT and bleeding times are normal in the patient with hemophilia B. Treatment options consist replacement therapy with prothrombin-complex, plasma-derived factor IX, and recombinant factor IX concentrates (57).

\section{Acquired Disorders}

In addition to the inherited disorders, there are acquired disorders of hemostasis that pose a significant bleeding risk (Table 3). Some of the more commonly described acquired bleeding disorders include acquired hemophilia, vitamin $\mathrm{K}$ deficiency, hepatic disease, disseminated intravascular coagulation (DIC), and immune thrombocytopenia purpura (ITP). Drug-induced bleeding disorders are by far the most prevalent. These patients may also have co-morbid illnesses that impact upon bleeding risk.

\section{Acquired Hemophilia}

Acquired Hemophilia A and B are consequences of autoimmune processes. Factor VIII or factor IX inhibitors may develop in the setting of pregnancy, drug-intake, or disease, such as lupus erythematosus, rheumatoid arthritis, or cancer $(59,60)$. Factor VIII auto-antibodies develop due to a dysregulation in the immune system (61). These autoantibodies interfere with several Factor VIII functions: binding to von Willebrand factor and phospholipids, interactions with Factor $\mathrm{IX}_{\mathrm{a}}$ and $\mathrm{X}_{\mathrm{a}}$, and the formation of Factor $\mathrm{VIII}_{\mathrm{a}}$-Factor IX $_{\mathrm{a}}$-phospholipid complexes (61).

Acquired hemophilias develop in 1 out of 1,000,000-4,000,000 patients (59). Mortality was high in these patients until the 1980s. Even since then, major bleeding has been reported in $87 \%$ of patients and the associated-mortality is still as high as $22 \%(62)$. Factor replacement therapy is not useful (63). Remission may be induced by steroids, immunoglobulins, plasmapheresis, and immunosuppression $(59,60,64)$.

Management continues to be a challenge (59). Acute bleeding may be controlled by using activated forms of factors VII, VIII, and IX, in order to bypass inactivated factors VIII and IX. Historically, prothrombin-complex concentrates or products containing factor VIII inhibitor-bypassing activity have been used. Recombinant activated factor VII has recently been approved. This product stops spontaneous bleeding and prevents excessive bleeding during complex surgical procedures (63). Other less successful treatment options include DDAVP, high-dose factor VIII concentrates, intravenous immunoglobulin, and immunosuppressive drugs, such as steroids, cyclophosphamide, cyclosporine, and rituximab $(59,61)$.

\section{Vitamin K Deficiency}

Microsomal carboxylase, a liver enzyme, converts clotting factors II, VII, IX and $\mathrm{X}$ to functionally active forms, but depends on the cofactor, Vitamin K. Carboxylation enables these factors, in turn, to bind with calcium (factor IV) and act as cofactors on platelet phospholipid membranes. Vitamin $\mathrm{K}$ is efficiently recycled in the liver by epoxide reductase (12).

When vitamin $\mathrm{K}$ deficiency occurs, the clotting factors, II, VII, IX and X, become depleted in an order that is determined by their individual half-lives. Factor VII has the shortest half life and is the first to be depleted. This is followed by factors IX and X and finally, factor II.
Without vitamin $\mathrm{K}$ these procoagulants are ineffective, even if there are sufficient amounts in the plasma. In addition, anticoagulant proteins $\mathrm{C}$ and $\mathrm{S}$ require similar enzymatic carboxylation for activation (11)

Endogenous intestinal bacterial flora continually synthesize vitamin $\mathrm{K}$. In patients, who have normally functioning livers, there is only a small requirement for exogenous vitamin $\mathrm{K}$, which is provided by the typical Western diet. Vitamin $\mathrm{K}$ deficiencies are commonly found in 1.Malnutrition; 2. Fat malabsorption syndromes, particularly biliary tract disease; 3. Broad spectrum antibiotic use leading to the destruction of endogenous bacterial intestinal flora; 4 . Neonates since they lack stores of vitamin $\mathrm{K}$ and can become deficient in this vitamin in absence of supplemental therapy; 5 . Diffuse liver disease (65).

One of the most prevalent causes of vitamin $\mathrm{K}$ deficiency is failure of the liver to secrete bile into the gastrointestinal tract which can occur as a result of biliary duct obstruction or liver disease: lack of bile prevents adequate fat digestion and absorption and therefore, prevents the absorption of a fat-soluble vitamin, such as vitamin K. Neonates may be deficient in Vitamin K, since bacterial flora might not have yet become established in their gut. In gastrointestinal disease, vitamin K deficiency occurs as a result of poor absorption of fats and the consequently poor absorption of vitamin $\mathrm{K}$. Liver pathology often causes decreased production of vitamin $\mathrm{K}$ and non-vitamin $\mathrm{K}$ dependent clotting factors. Even if vitamin stores are normal, hepatocyte dysfunction interferes with vitamin $\mathrm{K}$ dependent carboxylation of clotting factors (65).

In adults suffering from vitamin $\mathrm{K}$ deficiency or decreased synthesis of vitamin K-dependent factors, a bleeding diathesis may occur. This may be characterized by hematomas, hematuria, melena, ecchymoses, and bleeding from the gums. Due to this, Vitamin $\mathrm{K}$ is injected into all surgical patients with liver disease or with obstructed bile ducts prior to surgery. If vitamin $\mathrm{K}$ is given to a deficient patient four to eight hours before surgery and if the liver parenchymal cells are functioning at least at $50 \%$ of normal, sufficient clotting factors will be produced and excessive bleeding may be prevented during surgery. 


\section{Liver Disease}

Liver disease represents a continuum of disorders. Chronic liver disease can represent the endpoint of active chronic hepatitis or alcoholic liver disease or it can be the starting point, en route to hepatic failure or cirrhosis (66). All stages of liver disease increase the risk of bleeding. Even ethanol can suppress hematopoesis (67). Secondary hemostasis is most commonly thought to be affected by liver disease. Except for von Willebrand's factor and Factor VIII, both of which can be produced by endothelial cells or megakaryocytes, all remaining clotting factors are formed by the liver $(11,68)$. Decreased liver production of clotting factors and an increase in factor consumption affects secondary hemostasis. In reality, however, liver disease affects all three facets of coagulation: primary hemostasis, secondary hemostasis, and fibrinolysis (Table 4) $(11,69)$. Thrombocytopenia and several defects in platelet function are common. Increased fibrinolysis occurs in
$30 \%$ of patients with end-stage liver disease, thus placing these patients at a high risk for massive bleeding following invasive procedures.

Qualitative and quantitative deficits in clotting factors may develop (68). Early on, hepatic dysfunction may mimick vitamin $\mathrm{K}$ deficiency. First, a deficiency of factor VII (shortest plasma half-life) occurs. This is then followed by deficiencies in factors IX, X and II. The reduction in levels of vitamin $\mathrm{K}$ dependent factors correlates with the degree of liver disease (68). Progressive deterioration in liver function affects the remaining clotting factors: I, V, XI, and XII. The synthesis of anticoagulant factors, such as, antithrombin III, protein C and protein $S$ is also impaired, which can lead to thromboembolic events and the consumption of clotting factors. Clearance of activated clotting factors from circulation by the liver is diminished allowing constant activation of the clotting cascade $(11,68)$.

Thrombocytopenia and platelet

Table 4. Etiology of hemostatic abnormalities in the liver

\author{
Thrombocytopenia \\ Decreased production \\ hypersplenism \\ increased consumption \\ Impaired platelet function \\ decreased FDP clearance \\ Decrease factor synthesis \\ decreased hepatocyte function \\ vitamin $\mathrm{K}$ deficiency (diet, malabsorption) \\ Increased factor consumption \\ decreased clearance of activated factors \\ decreased synthesis of inhibitors (proteins $C$ and S) \\ Increased fibrinolysis \\ decreased synthesis of $\alpha 2$-antiplasmin \\ decreased clearance of t-PA
}

Adapted from: Petrovitch CT, Drummond JC. Hemotherapy and Hemostasis. In Barash PG, Cullen BF, Stoelting RK (eds). Clinical Anesthesia, 4th Ed, Lippincott Williams \& Wilkins, Philadelphia, 2001;224 (11) dysfunction impair primary hemostasis. A reduction in platelet numbers, in liver disease, is largely due to decreased thrombopoetin secretion and hypersplenism. Platelet counts may be reduced due to splenic sequestration in the setting of portal hypertension: the most common cause of thrombocytopenia in liver disease (68). Platelet counts may decrease to as low as $30-40,000 / \mathrm{mm}^{3}$ (68). In advanced liver disease, the pathogenesis of thrombocytopenia is more complex (68). Impaired platelet function results from advanced liver disease due to insufficient clearance of FDPs. Excessive FDPs in the plasma coat the surface of platelets and impair platelet aggregation, function and clot formation (11). The degree of platelet dysfunction parallels the severity of liver disease and roughly corresponds to the degree of serum bilirubin (68). Platelet function studies, however, may not predict bleeding tendency in liver disease (70).

Increased fibrinolysis occurs as a result of decreased clearance of tissue plasminogen activator ( $t-P A)$ from the circulation by the impaired liver and decreased hepatic secretion of $\alpha 2$-antiplasmin (11). The combined effects of increased fibrinolysis and persistent coagulation in patients with advanced liver dysfunction can result in continual low grade disseminated intravascular coagulation (DIC).

Pre-procedure screening should include a platelet count, hemoglobin, PT, aPTT, and fibrinogen level. The PT may be relatively more prolonged than the PTT (68). However, the measurement of specific clotting factor levels may be abnormal, even if the routine screening tests are normal (68). Reductions in Factor VII levels may be an early marker of parenchymal liver disease, whereas reduced Factor IX levels don't occur until advanced stages of liver disease. In centers where such tests are not readily available, strategies have been published to assist in diagnosing the correct liver disease (68).

Several management strategies for coagulopathy secondary to liver disease are present: vitamin $\mathrm{K}$ supplementation, fresh frozen plasma, plasma exchange, platelet transfusions, and cryoprecipitate infusion. Vitamin $\mathrm{K}$ replenishment may partially correct liver disease-associated coagulopathy, due to the reduced production of Vitamin K-dependent factors (70). 
In biliary tract disorders, such as biliary cirrhosis, Vitamin K may completely correct the coagulopathy. Typical doses of 10 $\mathrm{mg}$ are given subcutaneously on a daily basis and the PT is closely monitored (68). Fresh frozen plasma can be administered to correct markedly prolonged PT/aPTT times, by replacing all necessary clotting factors. FFP is particularly important in DIC. Repetitive infusions of $10-15 \mathrm{ml} / \mathrm{kg}$ every 12 hours are usually needed. Plasma exchange has the same effect as FFP, but the risk of volume overload is reduced. Platelets should be maintained above 70,000 to $80,000 / \mu \mathrm{L}$. A minimum level of 50, 000 is recommended for liver biopsies (68). The use of desmopressing to shorten bleeding time is used by some investigators (68). Cryoprecipitate infusions should be used if fibrogen levels are less than $75 \mathrm{mg} / \mathrm{dl}$ (68). One bag of cryoprecipitate may raise the serum concentration by $5 \mathrm{mg} / \mathrm{dl}$. Typically, 2 bags of cryoprecipitate should be given per $10 \mathrm{~kg}$

Table 5. Major disorders associated with Disseminated Intravascular Coagulation (DIC)

Obstetric Complications
Abruptio placentae
Retained dead fetus
Septic Abortion
Amniotic fluid embolism
Toxemia
Infections
Gram-negative sepsis
Meningococcemia
Rocky Mountain spotted fever
Histoplasmosis
Aspergillosis
Malaria
Neoplasms
Carcinomas of pancrease, prostate, lung, and stomach
Acute promyelocytic leukemia
Massive Tissue Injury
Traumatic
Burns
Extensive surgery
Miscellaneous
Acute intravascular hemolysis, snakebite, gaint heman

Acute intravascular hemolysis, snakebite, gaint hemangiomas, shock, heat stroke, vasculities, aortic aneurysm, liver disease

Adapted from: Cotran RS, Kumar V, Collins T. Red Cells Bleeding Disorders. In Robbins (ed). Pathology of Disease. 6th Ed. Philadelphia, 1999, 640.

of body weight and the fibrinogen should be maintained above $125 \mathrm{mg} / \mathrm{dL}$.

\section{Disseminated Intravascular Coagulation}

DIC represents the inordinate activation of the coagulation system (68). It is an acute, sub acute, or chronic thrombotic and hemorrhagic disorder that frequently occurs as a complication of numerous diseases. In the acute stage, patients present with multiple ecchymoses, mucosal and subcutaneous bleeding, visceral hemorrhage and tissue ischemia (71). Platelets and coagulation factors are depleted. The acute stage is life-threatening (71). Chronic DIC is subtle and less explosive. Chronic DIC demonstrates evidence of thromboembolism (71), such as superficial or deep venous thrombi. The coagulation system is activated, but factor levels and platelet counts may be increased, normal, or decreased (71).

Pathological activation of the extrinsic and/or intrinsic pathways of coagula- tion or impairment of clotting inhibiting factors may trigger DIC. Two major mechanisms are implicated. First, there is an excessive release of tissue factor (formerly thromboplastin) into the circulation. This may occur as a result of an amniotic fluid embolus, extensive soft tissue damage, or severe head injury. Secondly, widespread endothelial cell injury occurs and causes the release of even larger quantities of tissue factor, from fibroblasts. Broad endothelial cell injury may be produced by numerous processes such as deposition of antigen-antibody complexes, temperature extremes, or the presence of microorganisms (Table 5) (53).

DIC is characterized by the excessive formation of thrombin (factor $\mathrm{II}_{\mathrm{a}}$ ) throughout the vasculature and an activation of the coagulation cascade process. Accelerated clot formation leads to the formation of microthrombi that lead to tissue ischemia and the critical depletion of platelets and clotting factors. Concurrently, the fibrinolytic system is stimulated and plasmin is generated to lyse the fibrin clots. In addition, the mechanisms which normally serve to localize plasmin degradation of fibrin are overwhelmed and plasmin circulates unrestricted throughout the circulation. Fibrinolysis and circulating plasmin cause widespread degradation of both fibrin and fibrinogen. FDPs result in further inhibition of platelet aggregation, fibrin polymerization and have antithrombotic activity. All of these influences ultimately result in massive bleeding and the patient's demise. For instance, massive trauma results in a profound coagulopathy that is associated with thrombocytopenia, coagulation factor deficiency, and hypothermia (17).

Laboratory results that are characteristic of acute DIC include a prolonged PT and PTT. Factor V, Factor VIII, platelet, and fibrinogen levels are reduced and fibrin degradation product levels are elevated (71). In fact, The D-dimer test is the most specific test to assess for the presence of acute DIC (11). In chronic DIC the laboratory studies are more variable, except for FDP levels; FDP levels are elevated in both acute and chronic DIC. Treatment of underlying disease is the mainstay of DIC management (71). Acute DIC may be treated with factor, platelet, or fibrinogen replacement. Heparin and fibrinolysis-inhibitors, such as aminocaproic acid or tranexamic acid, may be used in acute DIC. Chronic DIC is primarily a hyper- 
coaguable state that may be managed with warfarin or low molecular weight heparin (71).

\section{Renal Disease}

Bleeding in uremic patients is typically mucosal, genitourinary, subdural, or gastrointestinal. Patients who are undergoing needle or catheter placement may be at significant risk of local, prolonged oozing and hematomas (54). Deep bleeding, such as an intramuscular hematoma, is uncommon (54). Hemostatic defects in renal disease are multifactorial (54). Several hypothesized mechanisms include defects in platelet and subendothelial metabolism, platelet-vessel interactions, and anemia $(54,69,72)$. Platelet function can be even more impaired in the presence of anti-platelet medications. The effects of anticoagulants that depend on renal clearance can be augmented. Low molecular weight heparins pose a particular problem: impaired clearance, prolonged half-life, and lack of reversibility with protamine can significantly increase bleeding risk in renal patients (69). In thromobocytopenic oncology patients, the presence of uremia did correlate with hemorrhagic risk but the absolute platelet count did not (73).

A comprehensive coagulation profile must be performed in renal failure patients that are pending an invasive procedure. The bleeding time is typically prolonged in uremia and although not ideal, may be predictive of bleeding $(54,72)$. Bleeding time is useful in monitoring response to therapy (69). Bleeding time, as compared to BUN or creatinine, is a better test in the assessment of bleeding risk in renal failure (54). Elevations in PT and a PTT denote the effect of other clotting problems, such as the lupus anticoagulant, or drugs, such as heparin (54). A variety of therapeutic modalities are available to correct renal dysfunction associated coagulation abnormalities prior to an invasive procedure (54). These include pre-procedure dialysis, abstinence from platelet active drugs, correction of anemia, desmopressin (DDAVP) $\left(0.3 \mu / \mathrm{kg}\right.$ over $\left.20^{\prime}\right)$, cryoprecipitate infusions, or estrogens. A 10 unit infusion of cryoprecipitate normalizes bleeding time in $50 \%$ of uremic patients (54). Conjugated estrogen infusions $(0.6 / \mathrm{mg} / \mathrm{kg} / \mathrm{d}$ X 5 days $)$ or oral estrogens shorten the bleeding time in uremia. The effect is rapid-within 48 hours and can last up to 10 to 14 days. Correction of anemia in renal failure also improves the bleeding time. Blood transfusion or the use of erythropoietin to raise the hematocrit above $30 \%$ appears to restore plateletvessel wall interactions.

\section{Thrombocytopenia and Idiopathic Thrombocytopenic Purpura}

True thrombocytopenia should be assessed prior to any invasive procedures. In the absence of platelet dysfunction, most individuals can tolerate invasive procedures with platelet counts about $80,000 / \mu \mathrm{l}$. Moderate thrombocytopenia with platelet counts in the range of 50,000 to $80,000 / \mu \mathrm{l}$ are usually asymptomatic but can result in significant bleeding depending on the invasive procedure. However, platelet counts as low as 58,000 and the associated bleeding time prolongation do not correlate with perioperative blood loss or transfusion requirements (74). Platelet counts in the range of 30,000 to $50,000 /$ $\mu \mathrm{l}$ will result in petechiae, easy ability to bruise, and excessive bleeding with minor procedures. Severe thrombocytopenia with counts below 10,000-20,000/ $\mu \mathrm{l}$ can result in spontaneous mucosal bleeding and potentially life-threatening bleeds $(43,75,76)$.

Immune thrombocytopenic purpura (ITP) is an acquired autoimmune disorder characterized by low platelet counts and mucocutaneous bleeding (77). This disorder occurs as a consequence of antibodies directed against platelet glycoproteins, such as GP IIb/IIIa (77). The antibody-coated platelets are removed from the circulation by the binding of the $\mathrm{Fc}$ moiety of the immunoglobulin to the Fc receptors on macrophages located predominantly in the spleen and liver (77). Chronic ITP is seen in adults. There is a female predominance and the most commonly afflicted are between the ages of 20 to 40 years old. Thrombocytopenia falls within a wide range of severity, but leukocytes and erythrocytes are typically normal. The thrombocytopenia may be clinically silent or associated with petechiae, easy bruising, or mucosal bleeding (14). The absence of a family history and systemic symptoms support the diagnosis of idiopathic thrombocytopenic purpura (77). Bone marrow aspiration may be performed in patients over the age of 40 with atypical features (77), but routine practice should be limited to those patients over the age of 60 (14).

Despite the common perception that
ITP has a benign course, even with platelet counts $<30,000$, a recent study refutes this (78). Based on mathematical models, pooled data from several clinical series, and the use of a fatal hemorrhage as an end-point, the five year mortality was $2.2 \%$ and $47.8 \%$ for patients with an age $<40$ or $>60$, respectively (78).

A variety of treatment modalities are available and the American Society of Hematology has recently established practice guidelines (14). In general, asymptomatic patients with platelet counts greater that $50,000 / \mu \mathrm{L}$ do not need medical intervention and can safely undergo minimally invasive procedures. This is dependent on these patients not receiving drugs that interfere with platelet function. Procedures are frequently performed on patients with thrombocytopenia of various etiologies with little risk of bleeding. The risk of hemorrhage in patients with coagulopathies may be more related to the experience of the medical personnel rather than the abnormality. In patients with platelets count less than $50,000 \mu \mathrm{L}$ who will be undergoing invasive procedures, some sort of medical intervention (platelet transfusions, corticosteroids, intravenous immune globulin, or anti-D immune globulin) may be required to raise the platelet count $(77,79)$. Very low platelet counts may require immunosuppressive therapy (77). Long term management may require a splenectomy (77). The management of these patients should be made in conjunction with a hematology specialist, since no single treatment algorithm is suitable for all patients (77).

\section{Factor XIII}

Routine coagulation studies (platelet function, prothrombin time, partial thromboplastin time, fibrinogen, antithrombin III) do not assess for Factor XIII deficiency (80). Low levels of factor XIII may interfere with fibrin monomer crosslinking and render clots more susceptible to degradation, but the clinical significance is not established. Acquired deficiency of Factor XIII may be present in several conditions: liver disease, inflammatory bowel disorders, peptic ulcer disease, and septicemia. Delayed post-operative hemorrhage is typical of Factor XIII deficiency since the early stages in the clotting cascade are not affected: hematomas may occur as long as 3-4 days after a neurosurgical procedure (80). 
DRUGS

\section{Antiplatelet Medications}

There are several types of antiplatelet agents with different pharmacological actions: cyclooxygenase inhibitors, adenosine diphosphate inhibitors, direct thrombin inhibitors, and GP IIb/IIIa receptor antagonists.

\section{Aspirin and Non Steroidal Anti-Inflam- matory Drugs}

Cyclooxygenase inhibitors inhibit the formation of thromboxane A2, which is responsible for vasoconstriction and secondary platelet aggregation. Platelets still adhere normally to subendothelium and form a normal, primary hemostatic plug. This fragile plug may be adequate for small vascular injuries, but not for stopping perioperative hemorrhage (224).

Aspirin is considered the 'reference standard' for antiplatelet agents by the American Heart Association. Aspirin is primarily used for the prevention and treatment of a variety of cardiovascular disorders and stroke. Aspirin is a potent cyclooxygenase inhibitor that suppresses thromboxane $\mathrm{A}_{2}$ production, but there are several mechanisms by which platelet aggregation is inhibited. Aspirin inhibits the enzyme phosphodiesterase and leads to increased levels of cyclic adenosine monophosphate (cAMP), an inhibitor of platelet aggregation. Aspirin also interferes with platelet function by antagonizing adenosine diphosphate (ADP) and glycoprotein IIb/ IIIa receptors $(11,53)$. Aspirin irreversibly inhibits cyclooxygenase for the life of the platelet, 7-10 days, but non-steroidal antiinflammatory agents only reversibly inhibit cyclooxygenase (81). This reversible inhibition normalizes within 3 days (81). Ketorolac transiently inhibits platelet function in healthy volunteers (82). The cyclooxygenase- 2 enzyme is induced in the presence of pain and inflammation, but is not present in platelets: selective cycloxygenase-2 enzyme inhibitors do not cause platelet dysfunction (83).

Increased doses of aspirin increase major bleeding risk (84). Aspirin therapy has been implicated as a risk factor in intracranial subdural hematomas (85). Preoperative anti-inflammatory consumption may increase bleeding during orthopedic surgery (86), prostate surgery (87), tonsillar surgery (88), and hysterectomies
(89). Others dispute whether aspirin use increases perioperative blood loss in during surgery $(27,90)$.

Arguably, the lack of reported major bleeding complications in association with aspirin or NSAID use does not guarantee the prevention of a future complication. Postponing a procedure may not be necessary in the setting of aspirin or NSAID use, but may be prudent in some cases. Theoretically, for patients with significantly prolonged bleeding times, $>15-$ 18 minutes, there are several options: postpone case, administer DDAVP until the bleeding time normalizes, or perform the procedure and administer platelet transfusions, if bleeding does develop.

\section{Thienopyridine Inhibitors}

Thienopyridine inhibitors interfere with adenosine diphosphate and affect both primary and secondary platelet aggregation; specifically, these agents irreversibly inhibit ADP binding to the platelet $\mathrm{ADP}$ receptor and this subsequently prevents activation of the GpIIb/GpIIIa complex, the major platelet receptor for fibrinogen (91).

Two common thienopyridine inhibitors include ticlopidine and clopidogrel. Ticlopidine and clopidogrel modulate vascular smooth muscle and interfere with platelet-fibrinogen, platelet-platelet, and platelet-vascular endothelium interactions (92-94). Steady state is achieved in 7 days for clopidogrel and 14-21 days for ticlopidine. The irreversible antiplatelet actions of ticlopidine may persist for 10 15 days after discontinuation (20). Clopidogrel is biotransformed by the liver, via the cytochrome p450 system. 2-oxo-clopidogrel is theorized to be the main intermediate metabolite that is a precursor to the final active metabolite $(95,96)$. The final active metabolite expresses the maximum anti-aggregating activity, via platelet-ADP inhibition $(95,96)$.

Thienopyridine derivatives prevent arterial and venous thrombosis (91). Ticlopidine and clopidogrel are more effective than aspirin in preventing further adverse vascular events following stroke, myocardial infarction, peripheral vascular disease, or vascular stent implantation $(91,92,97)$. Clopidogrel may have a therapeutic advantage over aspirin in those patients with arterial thromboembolic disease, in whom aspirin therapy is contraindicated or not effective (98). In fact, thienopyridine derivatives are often used in conjunction with aspirin, despite lack of safety data and the increased risk of hemorrhage. Recent investigations have demonstrated that the combination of clopidogrel with aspirin is more effective than aspirin alone and may be better tolerated than the combination of ticlopidine and aspirin, for the prevention of atherothrombosis following intravascular stent placement (99). Ongoing trials are assessing the efficacy and safety of clopidogrelaspirin combinations after acute ischemic coronary events and in patients at very high risk of stroke (99).

Bleeding time is prolonged to a greater extent with clopidogrel over aspirin, but absolute prolongation is significant with either agent alone (100). However, the greatest prolongation of bleeding time occurs with simultaneous use of aspirin and clopidogrel (100).

Purpura and epistaxis were reported to occur at a rate of $2.9-5.3 \%$ with clopidogrel $(92,97)$. Bleeding rates are similar among patients with atherosclerotic vascular disease that are receiving either clopidogrel or medium dose aspirin: 9.3\%. Rates of serious bleeding between clopidogrel and aspirin groups are $1.38 \%$ and $1.55 \%$, respectively. Serious hematological events, such as thrombocytopenic purpura, have occurred with ticlopidine, but are rare with clopidogrel. There is a significant increase in the risk of major and minor bleeding in patients receiving clopidogrel and aspirin versus aspirin alone for acute coronary syndromes. Major bleeding occurred $3.7 \%$ and $2.7 \%$ of patients with acute coronary syndromes that were receiving clopidogrel and aspirin (75-375 mg) versus placebo and aspirin, respectively (101). Most of the bleeding events were gastrointestinal or at the arterial puncture sites. In patients undergoing coronary artery bypass surgery, there was an increased rate of major bleeding between the clopidogrel and placebo groups, if clopidogrel was stopped within 5 days of surgery. Overall, the risk of bleeding with clopidogrel is comparable to aspirin, but lower than that with GpIIb/IIIa receptor antagonists (91, 101). The risk of intracranial bleeding is less with clopidogrel compared to aspirin (91). The excess risk bleeding of clopidogrel in patients receiving antiplatelet therapy for unstable angina is $1-1.2 \%$, which implies that the adjusted hazard ratio for major bleeding is $1.6(84)$. 


\section{Glycoprotein Receptor Antagonists}

Fibrinogen and von-Willebrand factor have multiple binding sites for platelet Gp IIb/Gp IIIa receptors. Antagonism of these receptors will interfere with the final common pathway of platelet aggregation and cross linking $(102,103)$. GP IIb/ Gp IIIa inhibitors are used synergistically with heparin and aspirin in the management of acute coronary syndromes and in percutaneous coronary interventions. Depending on the agent, the time required for normalization of platelet function varies between 8 to 48 hours following intravenous administration. Gp IIb/ IIIa antagonists pose a significant bleeding risk during this interval. There is almost a two-fold bleeding risk associated with Gp IIb/IIIa inhibitor use in patients undergoing percutaneous coronary interventions: 1.9 percent of these patients develop a clinically significant hemorrhage (104). The majority of patients receiving Gp IIb/IIIa antagonists develop some degree of bleeding $(102,103)$ and this risk increases in the elderly (105). Thrombocytopenia, with platelet counts as low as 20,000 , may develop in 1-2 \% of patients using these agents (102).

Use of these agents during cardiac and vascular surgery is associated with an increased incidence of perioperative bleeding $(106,107)$. Contraindications to the use of these agents include a history of surgery within 4-6 weeks. Interventional radiology guidelines suggest delaying elective surgery for 24-48 hours after abciximab and 4-8 hours after eptifibatide or tirofiban. Surgery performed within 12 hours of abciximab will necessitate a platelet transfusion (108).

Overall, the management of antiplatelet agents includes the use of platelet concentrates. Platelet concentrates prevent bleeding time prolongation in patients undergoing cardiac bypass surgery (74). The French Society of Anesthesiology and Intensive Care recommends using platelet transfusions, if increased surgical bleeding occurs in patients using antiplatelet agents (109).

\section{Dextran}

Dextran is not an anticoagulant, but is anti-thrombotic by virtue of hemodilution, plasma volume expansion, and modulation of the hemostatic system (110). Dextran inhibits thrombus formation. Dextran enhances the conversion of fibrinogen to fibrin. Since fibrin is functionally and structurally inferior to fibrinogen, fibrin clots are more vulnerable to lysis. This clinical effect occurs 2-8 hours after the infusion is started and depends on the dose and molecular size of dextran. Additionally, dextran reduces the level of factor VIII activator, which in turn, diminishes platelet adhesiveness and aggregation $(110,111)$. The typical use is for venous thromboprophylaxis for general and orthopedic surgery (110). Dextran has been used as a volume expander during surgery and may not significantly impair hemostasis for hemodilution below $30 \%(110,112)$. Dextran must not exceed $1.5 \mathrm{~g} / \mathrm{kg}$ of body weight $/ 24$ hours, in patients without hemostatic abnormalities $(110,112)$. Dextran is contraindicated in patients with inherited or acquired hemostatic abnormalities, such as hemophilia or thrombocytopenia (112).

\section{Warfarin}

Oral anticoagulants exert their anticoagulant effect by interfering with gamma carboxylation of vitamin K-dependent coagulation factors (113). The liver enzyme, epoxide reductase, is blocked and thus, the regeneration of reduced Vitamin $\mathrm{K}$ is prevented. The vitamin $\mathrm{K}$-dependent factors, II, VII, IX, X, S, and C, are functionally depleted $(65,113)$.

The intensity of warfarin therapy depends on the proportion of inactive factors and the duration of effect depends on factor half-life. If less than $40 \%$ of any particular factor is present, bleeding may occur (113). After administration of an effective dose of warfarin, the coagulant activity of the blood decreases to about 50 percent of normal by the end of 12 hours and to about 20 per cent of normal by the end of 24 hours. In other words, the coagulation process is not blocked immediately but must await the natural consumption of prothrombin and other affected coagulation factors already present in the plasma (65).

The prothrombin time and international normalized ratio are most sensitive to the activity of the factors with the shortest half-lives, VII and X, but least to the factor with the longest-half life, II. When factor VII activity is reduced to $55 \%$ of baseline, the INR will exceed 1.2. When factor VII activity is reduced to $40 \%$ of baseline, the INR will exceed 1.5. Hence, hemostasis is presumed to be normal when the INR is $<1.5$, since factor levels exceed $40 \%(5,113)$. The caveat is this applies to INR values upon initiation of warfarin therapy. This does not apply to the converse situation, recovery of hemostasis after stopping warfarin. Factor II and $\mathrm{X}$ levels will take longer to normalize and may not be adequate even if the INR $<1.4$ (114). Typically, however, an INR value that is within the normal range implies that there are sufficient levels of vitamin $\mathrm{K}$ dependent clotting factors (5) and coagulation returns to normal within one to three days after discontinuing warfarin therapy (65).

Warfarin is typically prescribed for patients at risk for arterial or venous thromboembolism (2): venous thromboembolism prevention and treatment, deficiencies of protein S and C, atrial fibrillation, prosthetic heart valves, and acute myocardial infarctions complicated by ventricular mural thrombi (11).

Several factors influence the response to warfarin: race, drug interactions, diet, advanced age, female gender, body weight, and pre-existing medical conditions such as liver or renal disease $(5,115)$. Age and the intensity of anticoagulation are known risk factors for intracranial and possibly, intraspinal bleeding $(116,117)$.

A warfarin overdose manifests as ecchymosis formation, mucosal hemorrhage, and subserosal bleeding into the wall of the gastrointestinal tract. The PT is markedly prolonged in the presence of a coumadin overdose. If an expansile hematoma occurs following an invasive procedure, in the presence of anticoagulation with coumadin, urgent care is warranted. Close monitoring of vital signs, prothrombin time, and hematocrit are imperative and surgical attention may be necessary (118). Administration of fresh frozen plasma and vitamin $\mathrm{K}$ are necessary to reverse the effects of warfarin (114, 118-120).

There is no consensus on the optimal management of patients receiving oral anticoagulants in the perioperative period $(2,120)$. Once the INR reaches 1.5 , certain types of surgery may proceed uneventfully (120). Kearon et al (120) estimates that if warfarin is held for four days prior to surgery and re-started the night of surgery, then the actual time frame, within which patients are exposed to thromboembolism risk, would only be one day prior and one day after surgery (120).

Dental procedures, joint and soft tissue injections, arthrocentesis, cataract 
surgery, upper endoscopy or colonoscopy with or without a biopsy can be safely performed in the presence of oral anticoagulation (2). Arthrocentesis and joint injections do not increase the risk of joint or soft tissue hemorrhage (121).

The dental literature advises holding coumadin for 3-4 days until the international normalized ratio decreases to the 1 2 range. If systemic heparinization is used in the interim then it should be stopped 6 hours prior to the procedure. Coumadin should be restarted in the evening following the procedure.

The American Society for Gastrointestinal Endoscopy indicates that for lowrisk procedures (eg. colonoscopy with or without biopsy) full dose anticoagulation should be continued. In patients undergoing high risk procedures, such as polypectomy or in those patients at high-risk for thromboembolic complications, perioperative heparinization should be administered and oral anticoagulation held (122). Nonetheless, even these recommendations may not be full adopted by gastroenterologists.

For other invasive and surgical procedures, oral anticoagulation needs to be withheld, and the decision to pursue aggressive perioperative anticoagulation with unfractionated or fractionated heparins depends on an individualized assessment of risk of thromboembolic versus bleeding events. When the risks of major disability following arterial or venous thrombosis versus those following postoperative hemorrhage are considered, perioperative management of oral anticoagulant becomes more complex. Recurrent venous thromboembolism can be fatal in $6 \%$ and can cause serious disability in $2 \%$ of patients, respectively (120). Arterial thromboembolic events are more serious: $20 \%$ of patients die and $40 \%$ of patients suffer serious disability (120). However, only $3 \%$ of post-operative hemorrhages are fatal (120).

The calculated risk of stroke and venous thromboembolism in patients that chronically receive, but stop oral warfarin prior to a surgical procedure is 0.6 and $0.3 \%$, respectively (2). These rates are greater than the expected stroke rates for patients with atrial fibrillation and mechanical valves that never receive anticoagulation (2). For instance, withholding coumadin for 4 days prior to surgery, in a patient with a mechanical valve, would expose the patient to a $0.4 \%$ risk of stroke.
The calculated stroke incidence for a patient, with a mechanical heart valve, who never receives any anticoagulation, over the same interval, would be $0.04 \%$ (2). Theoretical explanations for this include the hypercoaguable state induced by holding and restarting coumadin and the surgical milieu (2). Furthermore, death or permanent disability is common following arterial thromboembolism and infrequent following venous thromboembolism or post-operative bleeding (2).

Hence, guidelines based on the risk of stroke for certain conditions, assuming oral anticoagulation has never been administered, have been developed. If the risks and consequences of bleeding are high, then oral anticoagulation should be held irrespective of the annual stroke risk. The need to administer intravenous heparin or subcutaneous low molecular weight heparin when the INR becomes subtherapeutic depends on stroke risk. If the risk is $<4 \%$, eg. chronic atrial fibrillation without thromboembolic stroke or cardiomyopathy without atrial fibrillation, heparin therapy is not needed. If the risk is $4-7 \%$, eg. mechanical aortic valve, heparin therapy is optional. If the risk is $>7 \%$, eg. mechanical mitral valve or atrial fibrillation with a history of thromboembolic stroke, then heparin therapy is required (2).

Similar recommendations are provided by other authors. Kearon and Hirsh (120) suggest that warfarin should be held in patients whose INR is maintained between 2 to 3 and that the INR should drop below 1.5 , before surgery is started. Warfarin may have to be held longer if the INR is maintained above 3 or the requisite INR prior to surgery has to be $<1.3$. One day prior to surgery the INR should be measured and if the INR $>1.8$, vitamin K should be administered (120).

Several series have argued that oral anticoagulation can be safely withheld in patients with mechanical valves that undergo non-cardiac surgery $(123,124)$. Mechanical mitral valves, however, may need perioperative heparinization (123). Ananthasubramanian et al(125), withheld oral anticoagulation for a mean of $15+/-4$ days, in patients with mechanical heart valves, without any thromboembolic complications (125). In contrast, bleeding complications can increase if oral anticoagulation is not withheld or if perioperative intravenous heparin therapy is administered prior to surgery $(120,126$, 127). Immediate initiation of intravenous heparin therapy after surgery is likely to increase the risk of major bleeding (120). The calculated bleeding risk in the background of oral anticoagulation is $2-4 \%$ for major surgery and $0-2 \%$ for invasive procedures (2). Two days of intravenous heparin therapy will increase the absolute rate of major post-operative bleeding by 3 percent (120). One strategy, not yet validated in clinical trials, includes the use of outpatient subcutaneous low molecular weight heparin therapy as a bridge to surgery $(2,128)$.

In summary, recommendations for perioperative management of oral anticoagulation have been made based on the type of thromboembolic event (venous versus arterial) and specific risk factors for thromboembolism.

If surgery is planned within the first month after an acute venous thromboembolic episode, then the surgery should be post-poned. If this is not possible, warfarin should be held, and intravenous heparin should be administered whenever the INR falls below 2. Intravenous heparin should be held 6 hours prior to and restarted 12 hours after surgery, without a bolus. Heparin may have to be held even longer if there is bleeding at the surgical site. If oral anticoagulation has been administered for $>1$ month, but $<3$ months following venous thromboembolism, preoperative intravenous heparinization is not needed, unless the patient has additional risk factors. Post-operative heparinization is needed (120), however if oral anticoagulation has been administered for $>3$ months, only post-operative venous thromboembolism prophylaxis is necessary, but in combination with mechanical methods, e.g., pneumatic compression devices (120).

Elective surgery should be deferred in the first month following an arterial embolism. Essential surgery in this period should be managed with pre-operative heparinization and if the bleeding risk is low, post-operative heparinization. In patients receiving prophylaxis for the prevention of arterial thromboembolic events, e.g., mechanical heart valves or non-valvular atrial fibrillation, perioperative intravenous heparinization is not necessary (120) and should be avoided due the risk of post-operative bleeding. Subcutaneous unfractionated and low molecular weight heparin should be used for prophylaxis in hospitalized patients. No prophylaxis is needed for out- 
patients (120).

Watts and Gibbs (119) only agree with one scenario in these guidelines: patients with acute venous or arterial thromboembolism that have been treated with oral anticoagulation therapy for less than one month. All other scenarios would require perioperative low molecular weight heparin therapy (119), with doses that range between $20 \mathrm{mg} /$ day to $1.5 \mathrm{mg} / \mathrm{kg} / \mathrm{day}$. The dose depends on the risk of arterial or venous thromboembolism recurrence, whether the risk is high, e.g., malignancy, valvular atrial fibrillation, and caged-ball prostheses, or nonexistent (119).

\section{Thrombolytics and Fibrinolytics}

Thrombolytic drugs, such as urokinase and tissue plasminogen activator, lyse pathological thrombi and hemostatic plugs. The major risk of these agents is hemorrhage (20). Resolution of the action of thrombolytic drugs may take days: plasmin dissolves fibrin clots and forms fibrin degradation products, which inhibit platelet aggregation. Some contraindications to thrombolytic therapy include surgery within ten days, including organ biopsy and puncture of non-compressible vessels such as epidural veins $(5,129)$.

\section{Heparin}

Heparin is a negatively charged, water-soluble glycosaminoglycan and clinically, the most important antithrombotic drug (130). Heparin is widely used for anticoagulation in vascular surgery and in procedures requiring cardiopulmonary bypass. Standard, i.e., unfractionated heparin has a heterogeneous range of molecular weights (5-30,000 Daltons), due to the variable length of attached polysaacharide chains $(131,132)$. Anticoagulant and pharmacokinetic properties are correspondingly variable $(131,132)$. Low molecular weight heparins consist of glycosaminoglycans with lower, average molecular weights (4000-6500 Daltons). Low molecular weight heparins have relatively shorter polysaacharide chains, compared to unfractionated heparins. These differences in physical characteristics account for the different activities of low molecular weight versus unfractionated heparins.

One-third of standard heparins contain a specific pentasaccharide with a high affinity for anti-thrombin III (131133). This binding accelerates the activi- ty of antithrombin III, an ordinarily slow acting protease inhibitor. The heparinATIII complex results in a conformational change that significantly increases ATIII inhibitory activity. Heparin-antithrombin III binding inactivates thrombin (factor $\mathrm{II}_{\mathrm{a}}$ ), factor $\mathrm{X}_{\mathrm{a}}$, factor $\mathrm{IX}_{\mathrm{a}}, \mathrm{XI}_{\mathrm{a}}$, and $\mathrm{XII}_{\mathrm{a}}$ $(20,130)$. Factor $\mathrm{II}_{\mathrm{a}}$ is the most sensitive to inhibition $(20,131,132)$. Heparin-antithrombin III complexes further inhibit a procoagulant feedback loop via Factors $\mathrm{V}$ and VIII. The relative activity of heparin depends on the number and size of molecules containing the pentasaacharide sequence. Whereas higher molecular weight heparins inactivate both factors $\mathrm{X}_{\mathrm{a}}$ and IIa, lower molecular weight heparins only inhibit $\mathrm{X}_{\mathrm{a}}(131,132)$.

Heparin must be given parenterally, since the oral bioavailability is zero. Intravenous injections result in an immediate anticoagulant effect, but subcutaneous injections have a delay of 2 hours. This anticoagulant effect is dose and molecularsize dependent $(131,132)$. The anticoagulant effect, furthermore, is non-linear with increasing doses. This has important clinical applications. Practitioners often think the half-life of heparin is on the order of 30-60 minutes and these values may be valid at common clinical doses: 25-100U/kg $(131,132)$. However, at doses as high as $400 \mathrm{U} / \mathrm{kg}$, the half-life may increase to 150 minutes $(131,132)$.

Up to five percent of patients receiving heparin therapy will have a generation of circulating antibodies that bind a heparin-platelet factor complex on the surface of platelets or endothelium. This eventually results in platelet activation or endothelial cell injury and a subsequent prothrombotic state. This syndrome can be circumvented by the use of specially manufactured low-molecular weight heparin preparations, which retain anticoagulant activity but do not interact with platelets. Osipova et al (134) studied 17 patients that underwent extensive microsurgical autotissue graft repair and received prophylactic and therapeutic treatment with various antithrombotic, rheological, and antiischemic agents. The protocol's proposed combination of fraxiparin, a low molecular weight heparin, ketoprofene (ketonal), a nonsteroidal anti-inflammatory drug, perfluorane, and antikinnigen contrycal created optimal conditions for maintaining blood supply and oxygenation of the transplant. The regimen reduced blood clotting and platelet aggre- gation (134).

Significant bleeding has been reported in association with different types of heparin therapy. Heparin overdose manifests as subcutaneous hemorrhage and deep tissue hematomas. This anticoagulant is inactivated in the liver and is excreted by the kidneys, explaining the prolonged anticoagulant effects of heparin in the patient with hepatic or renal disease. Decreased body temperature is also associated with an enhanced anticoagulant effect of heparin. The PT and PTT are prolonged, but bleeding time is normal. Protamine may reverse the effect of heparin.

Low dose subcutaneous heparin, 5000 units B.I.D., is used for venous thromboprophylaxis in surgery and urology $(5,135)$. A common belief is that subcutaneous mini-dose heparin does not affect the activated partial thromboplastin time or platelet count. A therapeutic level, for arterial and venous thromboembolic disorders, is 1.5 times the baseline aPTT value. Mini-dose heparin can result in a 10 -fold variation in serum heparin concentrations (136). Ten to fifteen percent of patients will have measurable changes in aPTT and 2-4\% will become therapeutically anticoagulated $(136,137)$. Rarely, these changes can even persist for 5-6 hours after the dose (136). Platelet counts can reduce with subcutaneous mini-dose heparin, if given for greater than five days (131, 132). Routine monitoring of the aPTT is not necessary, unless there is concern. A platelet count should be checked in patients receiving prolonged mini-dose heparin.

Therapeutic anticoagulation for arterial thrombotic disease usually requires an elevation of aPTT to 1.5-2 times normal. Heparinization, even with a target activated partial thromboplastin time that is 1.5-2 times normal, is associated with an increased risk of spontaneous or induced bleeding (138).

\section{Low Molecular Weight Heparin}

Low molecular weight heparin, as compared to unfractionated heparin, has a longer half-life, has a more predictable bioavailability, lacks adequate tests to monitor the anticoagulant effect, cannot be reversed with protamine, and has a reduced influence on platelet function $(5,130,139,140)$. Protamine is the least toxic and most commonly used antidote to heparin $(130,139)$. Protamine may neutralize the antithrombin effects of low 
molecular weight heparin, but not its antifactor $\mathrm{X}_{\mathrm{a}}$ activity $(130,143)$. Prolonged use may be associated with an accumulation of anti- $\mathrm{X}_{\mathrm{a}}$ activity, dose-dependent fibrinolysis, and interference with platelet-endothelial binding $(144,145)$.

Two to four hours after subcutaneous administration, therapeutic levels of LMWH are reached and twelve hours after administration, $50 \%$ of peak levels are maintained (145). Enoxaparin, a LMWH has an elimination half-life of 2-3 hours, but the antithrombotic effect can last as long as 24 hours (132). Anti-hemostatic effects are magnified in the presence of advanced age, abnormal renal function, and concomitant NSAID use $(132,145)$.

Low molecular weight heparins significantly reduce the risk of venous thromboembolism following total hip replacement surgery, when compared to placebo and unfractionated heparin $(146,147)$. Low molecular weight heparins may be as safe and effective, in terms of major bleeding risk and thromboembolic event reduction, respectively, as unfractionated, adjusted dose heparin (128). Low molecular weight heparins reduce mortality after an acute deep venous thrombosis (128).

European guidelines for DVT thromboprophylaxis are $20 \mathrm{mg}$ once-daily for low risk and $40 \mathrm{mg}$ once-daily for high risk patients. Thirty or forty milligrams, twice daily are advised for the treatment of deep venous thrombosis (5). Enoxaparin was the first low molecular weight heparin approved thromboprophylaxis for major joint replacement surgery in the USA in 1993.

The uses of LMWH have expanded beyond the FDA-approved use. 'Bridging therapy' has been used for patients chronically anticoagulated with warfarin: prosthetic valves, parturients, chronic atrial fibrillation, and hypercoaguable states (148). LMWH has been used as therapy for acute deep venous thromboembolism, coronary syndromes, and preservation of graft patency following peripheral vascular revascularization procedures $(149,150)$.

\section{Herbal Medications}

Garlic, ginkgo, and ginseng are herbal medications that may cause bleeding. Garlic irreversibly inhibits platelet aggregation in a dose dependent fashion and may potentiate the antiaggregatory actions of non-steroidal anti-inflammatory drugs (151). Garlic has fibrinolytic activity (152). Ginkgo inhibits platelet activating factor (153) and has been implicated in several cases of spontaneous intracranial bleeding (154-156). Ginseng inhibits platelet aggregation and interacts with warfarin. Ginseng may prolong thrombin time and activated partial thromboplastin time $(5,157,158)$. Apart from the common herbal medications, there are others that may cause bleeding: feverfew, green tea, horse chestnut, cat's claw, ginger, chamomile, and fenugreek seed. Herbal preparations are not as tightly regulated as prescription medications. Government protections may not be sufficient to protect against adverse events. A significant proportion of patients presenting for a pre-anesthetic evaluation self-administer nutraceutical agents (159). Patients may not even report the consumption of herbs to practitioners (159).

\section{New Anticoagulants}

Some thrombi may continue to grow despite anticoagulant therapy. Fibrinbound thrombin is protected from inhibition by heparin and remains enzymatically active: bound fibrin can amplify its own generation through a positive feedback loop via coagulation factors $\mathrm{V}$ and VIII (160). Bound thrombin also continues to activate platelets through thromboxaneA2-independent mechanisms that cannot be blocked by aspirin (160).

Direct thrombin inhibitors (direct factor IIa inhibitors), unlike heparins (indirect factor IIa inhibitors), interact directly with thrombin, in both clot-bound and circulating forms (161). Unfractionated and fractionated heparins block circulating, but not clot-bound thrombin $(76,161)$. These agents are used in acute coronary syndromes and coronary angioplasty procedures, in patients with heparin induced thrombocytopenia, and in venous thromboembolism prophylaxis for hip surgery. One recent meta-analysis found that direct thrombin inhibitors are superior to heparin for the prevention of death or myocardial infarction in patients with acute coronary syndromes (160).

Direct thrombin inhibitors have an anticoagulant response that is more predictable than unfractionated heparins (161) and this response can be monitored by the aPTT. Thrombin inhibitors can block either thrombin's active site (univalent) or both the active and substrate recognition sites (bivalent) (161). The pro- totypical thrombin inhibitor is hirudin, which is bivalent, potent, and almost irreversible. Thrombin inhibitors have short half-lives ranging from 30 to 60 minutes (161). Hemorrhage may be life-threatening and the antithrombin effects cannot be reversed pharmacologically (5). Hirudin derivatives pose an increased risk of major bleeding compared to systemic heparin therapy (160). Spontaneous intracranial bleeding has occurred. Argatroban, another direct thrombin inhibitor, resulted in a higher incidence of major bleeding compared to historical controls: episodes of gastrointestinal and genitourinary bleeding and episodes requiring transfusions were higher with argatroban (162). Additional clinical experience is needed to assess bleeding risk (5).

Fondaparinux is a new selective factor $\mathrm{X}_{\mathrm{a}}$ inhibitor (163). This synthetic pentasaacharide has a half-life of 21 hours and a bioavailability approaching $100 \%$. Other beneficial pharmacological properties include the absence of metabolism or non-specific binding. Clinically this implies that dosing can be once-daily and that anticoagulation is more predictable. Fondaparinux has been approved for venous thromboprophylaxis following orthopedic surgery. Fondaparinux may reduce this risk by $50 \%$ more than low molecular weight heparins (163).

Fondaparinux and low-molecular weight heparins have similar bleeding risks and similar FDA warnings (163). A spinal hematoma has been reported in patients receiving twice the recommended thromboprophylaxis dose $(163,164)$. There were no additional hematomas in a prospective trial of 3600 patients.

\section{Hemostatic Drugs}

Hemostatic drugs deserve brief mention due to their potential role as therapeutic agents for acquired and congenital coagulation disorders and for major bleeding episodes.

Synthetic amino acids, such as aminocaproic acid and tranexamic acid, may interfere with fibrinolysis by reversibly binding to plasminogen and preventing its transformation to plasmin (165). Tranexamic acid is more potent than aminocaproic acid and has a longer half-life. These agents can reduce blood loss in primary menorrhagia, gastrointestinal bleeding, urinary tract bleeding, oral bleeding in hemophiliacs, oral bleeding following dental extractions in patients receiv- 
ing oral anticoagulant therapy, cardiac surgery, thrombocytopenia, patients receiving thrombolytics, joint replacement, and liver transplants (165). The dose of tranexamic acid is typically $10-15 \mathrm{mg} / \mathrm{kg}$. Side effects are typically dose dependent. The main risk, however, is a thrombotic complication.

Aprotinin is a polypeptide that reversibly inhibits the action of several serine proteases (165): aprotinin inhibits the coagulation cascades and fibrinolysis. Aprotinin does not affect platelets. It is primarily used in liver transplantation and cardiac surgery. Hypersensitivity reactions are common with this agent. Aprotinin may cause arterial or venous thrombosis, but several studies have failed to demonstrate this complication.

Desmopressin, an analogue of vasopressin, may temporarily boost factor VIII and von Willebrand factor levels in patients with acquired and congenital bleeding disorders (165). Desmopressin is useful when an immediate effect on hemostasis is required. Adverse events include facial flushing, headaches, water retention, and hyponatremia.

Conjugated estrogens shorten bleeding times in patients with uremia (165). The mechanism of action is unknown, but can last for two-three weeks. These agents may be useful in preventing bleeding during elective procedures or recurrent bleeding gastrointestinal or nasal bleeding episodes.

It is unclear if these agents will be useful in the management of bleeding complications following interventional pain procedures. Nonetheless, knowledge about ways to obtain hemostasis in face of a coagulation disorder is useful.

\section{Bleeding Complications in Interventional Pain Practice with and WITHOUT ASSOCIATED COAgUlOPATHY}

We have summarized the relevant literature on bleeding complications with respect to specific techniques and coagulopathies. We performed a literature search on MEDLINE, using the National Library of Medicine PubMed data base and the aid of an experienced medical librarian. A broad search strategy was used to capture all articles pertaining to bleeding as a complication in interventional pain and regional anesthesia. Over 2400 articles were identified, but only about one hundred and eighty were relevant. Additional data was obtained from references in these articles. The majority of articles pertained to neuraxial anesthesia, retrobulbar blockade, and intracranial hematomas. There were a few papers concerning lumbar plexus, lumbar sympathetic and peripheral nerve blocks. Some papers may have been missed due to the limitations of the search engine: the search engine can only identify terms listed in the citation, such as the title, authors, abstract, publication type, and MeSh terms. For instance, a paper that discusses the efficacy of a technique, but incidentally reports complications in the discussion may have been missed. A case report that describes the consumption of an anticoagulant by a patient, in the methods section, might have been missed. Nonetheless, we feel that these articles are representative: reported bleeding complications are rare and available for only a few commonly practiced interventional pain and regional techniques.

\section{Acquired And Congential Coagulation Disorders}

\section{Acquired and Congenital Hemophilias}

Three patients with Hemophilia A developed spontaneous spinal hematomas (166-168) that only required conservative treatment. In two patients, cervical epidural hematomas developed following minimal trauma (166). Factor VIII replacement therapy was initiated and both patients had dramatic improvements in neurological symptoms over several days; in one patient the hematoma was completely resorbed $(166,167)$. In another patient, the hematoma developed after sit-ups and spanned the segments C2T12 (168). Recombinant factor $\mathrm{VII}_{\mathrm{a}}$ was used and the hematoma resolved within 4 weeks (168).

Recombinant Factor VII $_{\mathfrak{a}}$ has been used to control surgical bleeding in nonhemophiliacs (58). As a 'universal' hemostatic agent, recombinant Factor $\mathrm{VII}_{\mathrm{a}}$ has controlled trauma-associated, post-surgi$\mathrm{cal}$, and spontaneous bleeding (58). Factor $\mathrm{VII}_{\mathrm{a}}$ has been used in neurosurgical patients with a pre-existing coagulopathy: anticoagulation, liver disease, and hemodilution (169). Normalization of coagulation status may occur within 20 minutes (169).

Unlike the previous three cases, surgical management may be required in some cases of hemophilia. A spontaneous cervical epidural hematoma developed in a patient with a deficiency of Factor XI. Despite continuous infusions of fresh frozen plasma and Factor XI cryoprecipitate therapy, an emergency decompressive laminectomy had to be performed, due to progressive neurological dysfunction. Post-operatively, the patient made a good recovery (56).

Published reports on the use of regional anesthetic or interventional pain procedures in hemophiliacs are few. Dhar (55) et al reported successful placement of an epidural catheter in a patient with Hemophilia A that developed spontaneous rupture of membranes. Recombinant factor VIII replacement therapy raised her factor VIII levels to $101 \%$ of normal, prior to catheter placement and was continued for 48 hours post-delivery. Intermittent recombinant factor replacement was continued for six weeks. There were no complications to the patient or her child (55). Elevations in coagulation factors during pregnancy, such as the $200-500 \%$ increase in factor VIII, may have also been protective in this case $(55,170)$.

Kang et al (171), reported successful use of a continuous axillary brachial plexus block, using a nerve stimulation technique, in a severe hemophilic. The patient had Factor VIII levels of less than $1 \%$ and developed severe hemophilic arthropathy of the elbow. He underwent radial head resection, synovectomy, and contracture release. No bleeding or neurological complications developed, despite some difficulty in keeping factor VIII levels above $30 \%$. The authors speculated that if Factor VIII levels were kept above 30\%, the risk of bleeding complications with a continuous axillary brachial plexus block should be equivalent to patients without hemophilia (171).

Epidural catheterization was used for labor in a patient who had two prior uneventful vaginal deliveries with epidural anesthesia. The epidural dressing became saturated with blood, five hours after placement. Coagulation studies were performed and the aPTT was 57 seconds (reference range: $25-38$ seconds). Epidural analgesia and anesthesia were continued. Cryoprecipitate was used well into the case, but the aPTT did not significantly change. Epidural site bleeding continued at a rate of $150 \mathrm{cc} /$ hour and hematuria spontaneously developed. Further coagulation studies demonstrated factor VIII activity of $4 \%$ and a presumptive diagnosis of acquired Hemophilia secondary 
to factor VIII inhibitors was made. Factor VIII concentrates and steroids were then administered for several days. The authors speculated that a caesarean section and emergent neurosurgical decompression were luckily averted, both of which could have lead to the patients demise.

These authors cautioned that abnormal coagulation studies in an otherwise asymptomatic patient should not be ignored (59). For example, an elevation of the aPTT may indicate a range of bleeding disorders: acquired hemophilia with inhibitors, congenital hemophilia, or von Willebrand disease. However, severe von Willebrand disease may be less severe than acquired hemophilia due to the presence of factor inhibitors (59).

\section{Von Willebrand Disease}

A spontaneous, spinal hematoma spanning 12 segments has been reported in a patient with von Willebrand disease (172), who also had spontaneous bleeding at other sites. There have been a few reported cases of safe epidural catheterization in patients with von Willebrand's disease $(52,170,173)$. Epidural catheterization was uneventful in one patient, whose vWF:Ag and Ricof levels were 28 and 42\%, respectively (52). In another report, the vWF:Ag level was 35\% and the Ricof level was $10 \%$ (173) and epidural catheterization was also uneventful.

\section{Thrombocytopenia and Idiopathic Thrombocytopenic Purpura}

A platelet count of 100,000 is abnormal and represents a value that is two standard deviations below the mean (174). Epidural catheterization is considered safe in patients with platelet counts $>100,000$ (175). Neuraxial procedures in patients with platelet counts less than 100,000 are controversial.

Waldman et al (176) prospectively performed 336 caudal epidural injections, with a 25 gauge needle, in 56 coagulopathic patients. Thirty seven patients had abnormal coagulation parameters, with a PT or aPTT that was 1.5 times the control value. Nineteen patients had thrombocytopenia with platelet counts $<50,000 /$ $\mathrm{mm}^{2}$. There were no spinal hematomas, but two patients had a small hematoma at the injection site (176). He concluded that thrombocytopenia and anticoagulation may only be relative contraindications to caudal epidural procedures.

Fifty-five to sixty-six percent of an- esthesiologists, depending on practice setting, would perform an epidural in a parturient that has a platelet count that ranges from 80,000 to 100,000 . Only a minority of anesthesiologists would place epidural catheters at lower platelet counts (177). One retrospective study identified 30 parturients that received epidural anesthesia with platelet counts ranging between 69-98,000 and 22 parturients with initial platelet counts $>100,000$ that subsequently dropped below 100,000 (178); none of the patients developed neurological complications (178). Rasmus et al (179) retrospectively identified 14 parturients with platelet counts less than $100,000(15,000-$ $99,000)$ that received epidural anesthesia without sequelae. However, their small sample size only ensured a $95 \%$ probability that the incidence of epidural hematoma would be less than $17 \%$ (180).

In parturients with thrombocytopenia, a history and physical consistent with bleeding is a contraindication to regional anesthesia. If there is no clinical evidence of bleeding, a platelet count should be repeated to ensure no further decrease in value, prior to the regional anesthetic. No cut-off value for the platelet count is advised and platelet function analyses are not recommended (181). If a regional block is going to be used then a spinal is preferable to an epidural; if an epidural is used then the local anesthetic concentration should be minimized to permit neurological evaluation (181). If a spinal must be used and profound thrombocytopenia is present, a platelet transfusion is advised. Platelet transfusions are advised for patients undergoing lumbar puncture, when their platelet count is less than 20,000 (182).

Spinal anesthesia was performed on a young parturient with idiopathic thrombocytopenic purpura, based on a negative history of bleeding diathesis and a normal bleeding time. However, this patient had a platelet count that varied between 46,000 to $64,000 /$ microliter. Despite proceeding with the procedure and an uneventful post-partum course, the authors expressed caution, i.e., spinal anesthetics should be limited to patients with platelet counts greater than 50,000/ microliter (183).

\section{Liver and Renal Disease}

The presence of even mild liver dysfunction may be a causal factor in procedure-related bleeding. Hepatic dysfunc- tion with accompanied portal hypertension may cause epidural venous congestion and increase the likelihood of bleeding (184-186). More advanced liver disease increases the risk of neuraxial hematomas following regional procedures: reduced production of clotting factors, portal hypertension induced epidural venous engorgement, and hypersplenisminduced platelet sequestration $(67,187)$. A spinal hematoma, following a lumbar puncture, has been reported in a patient with liver disease (188). An epidural hematoma following epidural catheter placement has been reported in patients with mild (174) and advanced liver disease (187)

A subarachnoid hematoma complicated by paraplegia has been reported as a complication of spinal anesthesia, in a patient with chronic renal failure (189). Although there are several reported cases of spontaneous epidural hematoma in patients with renal failure $(190,191)$, there are fewer reported cases following neuraxial anesthetics (192). Despite a negative history of bleeding and normal coagulation studies, a patient with chronic renal failure developed a delayed epidural hematoma, after epidural catheter placement for post-operative analgesia (192). Treatment options for a spinal hematoma in chronic renal failure patients include decompressive surgery, fresh frozen plasma, or desmpressin (190-192).

\section{Specific Anticoagulants}

Anticoagulation increases the absolute risk of bleeding. Warfarin is responsible for spontaneous bleeding in 3\% and $7 \%$ of patients, depending on whether the INR is $2-3$ or $>4$, respectively. The risk of bleeding is less than $3 \%$ when patients receive subcutaneous, intravenous or low molecular weight heparin (138). Thrombin inhibitors may induce bleeding in 2$5 \%$ of patients $(160,162)$. Thrombolytic therapy, however, presents the greatest risk of bleeding: 6-30\% (193). Invasive procedures or surgery may pose an even greater risk of significant bleeding in the face of anticoagulation.

Hittelet et al (194) provided a strategy for the management of anticoagulation during endoscopy. For low risk procedures, such as, upper endoscopy with biopsy, colonoscopy with biopsy or endoscopic retrograde and cholangiopancreatography with stent insertion (but without sphincterotomy), anticoagulation did 
not have to be adjusted. For high-risk procedures, such as polypectomy, endoscopic sphincterotomy, laser therapy, mucosal ablation and variceal treatment, anticoagulation would have to be adjusted. Warfarin should be discontinued four to five days before the procedure and restarted the night of the procedure. In weighing the risks vs. benefits, in terms of preventing bleeding versus thromboembolism, patients may require Vitamin $\mathrm{K}+/$ fresh frozen plasma or intravenous heparinization, respectively. Low molecular weight heparin may be an alternative to unfractionated heparin. Aspirin and nonsteroidal anti-inflammatory drugs do not have to be discontinued. Thienopyridine drugs should be discontinued 7-10 days prior to a high risk procedure (194).

Bleeding risks for procedures commonly performed by one specialty may not apply to those performed by other specialties. In patients undergoing cataract surgery, retrobulbar hemorrhage is more common in those patients who cannot stop anticoagulation and even in those who stop it prior to surgery, compared to those who have never been anticoagulated (195). Nonetheless, retrobulbar blockade may be safe in the presence of anticoagulation (196-198).

The administration of a neuraxial anesthetic in the presence of anticoagulation is a cause for concern: if a blood vessel is traumatized during the performance of a regional anesthetic, then a delayed hemorrhage may lead to a spinal hematoma and spinal cord compression (199).

Regional blocks are typically contraindicated in the presence of systemic anticoagulation with heparin, coumadin, and thrombolytics. Antiplatelet and unfractionated, low dose, subcutaneous heparin therapies are considered to present a very small risk of epidural hematoma. Low molecular weight heparin, however, was associated with a rise in the number of epidural hematomas and thus, prompted an FDA advisory (200).

The relative risk of bleeding in the presence of anticoagulation can be calculated. Stafford-Smith et al (201) assumed a baseline risk of one for neuraxial procedures that are performed without anticoagulation and without technical difficulties. The relative risk in the presence of aspirin therapy was no different when compared to patients that did not consume aspirin. Traumatic insertion during a neuraxial procedure increases the relative risk to eleven. Traumatic insertion, followed by intravenous heparinization, maximally increases the relative risk to 111 . Aspirin and intravenous heparin therapy, together, increase the risk to 26 . Intravenous heparin therapy that is administered within one-hour of the neuraxial block will increase the risk to 25 , compared to 2 , if heparin is delayed more than 1 hour (201). Fortunately, spinal hematomas following neuraxial anesthesia are rare. In a series of 17,733 neuraxial blocks, 3 spinal hematomas were identified in patients with abnormal hemostasis (202).

The American Society of Regional Anesthesia developed guidelines for the safe practice of neuraxial anesthesia in the presence of anticoagulation $(5,203)$. These may be the most relevant for interventional pain practice. The main quandary in developing these guidelines was the lack of data. Hence, the ASRA guidelines represent the opinions of experts on anticoagulation and regional anesthesia and are based on the available clinical and basic science literature $(5,204)$. Additionally, there are published European guidelines $(3,4)$ and several exhaustive reviews on this subject $(20,129,133$, 205, 207-210).

\section{Antiplatelet Medications}

\section{Aspirin and Non Steroidal Anti-Inflam- matory Drugs}

Aspirin and NSAIDs are not contraindications to neuraxial anesthesia (209). This issue is debated. Spanish guidelines on the use of antiplatelet agents with neuraxial procedures are more conservative than the American and German guidelines: hold these medications prior to neuraxial blocks (3). One survey in Europe showed that patients receiving aspirin therapy may be accepted for neuraxial blocks only after meeting some criteria: laboratory tests are within normal limits, aspirin is withheld for a minimum time period, and the dose should not exceed a maximal limit (20).

Vanderemeulen (208) identified two hematomas related to aspirin and indomethacin usage in a series of 61 patients. Aspirin has been implicated as the etiology in a few reported cases of spontaneous spinal hematoma (211-214). Ketorolac has the potential to worsen platelet function during spinal anesthesia (215) and has been implicated in two cases of epidural hematoma $(216,217)$. Indomethacin has been implicated in the development of a cervical epidural hematoma following repeat cervical epidural steroid injections (218). The combined use of naproxen and diclofenac has been implicated in the development of a large epidural hematoma following a subarachnoid puncture (219). MacDonald suggests withholding aspirin for 7-10 days and obtaining a bleeding time prior to epidural catheter placement (220). The main concern was how much bleeding could occur due to prolongation of bleeding and would such a volume of blood be significant to cause and extradural hematoma (22). Nonetheless, these few reports in the context of widespread use of cyclooxygenase inhibitors, suggests that neuraxial anesthesia can be safely performed in patients, receiving these agents.

In a combined series of 4714 patients consuming cyclooxygenase inhibitors, there were no hematomas (209). There were no adverse neurological events in 1422 high risk obstetric patients that received $60 \mathrm{mg}$ of aspirin, while participating in the collaborative low dose aspirin study in pregnancy (CLASP) (221). Recently, in a prospective series of 1035 individuals undergoing 1214 epidural steroid injections, fifteen percent of patients reported a history of bleeding and bruising and 32\% were taking non-steroidal anti-inflammatory medications. Platelet counts were performed on only $77 \mathrm{pa}-$ tients and all were greater than 100,000. $5.2 \%$ of patients developed minor hemorrhagic complications, but none developed a hematoma. NSAIDs did not increase the frequency of minor bleeding, but increased age, large needle gauges, type of needle approach, needle insertion at multiple interspaces, number of needle passes, volume of injectate, and accidental dural puncture were significant risk factors for bleeding (222).

In a retrospective series of over 1000 patients, Horlocker (223) reported that $39 \%$ were taking at least one antiplatelet drug and $11 \%$ were taking multiple antiplatelet drugs at the time of neuraxial block; no patient developed a spinal hematoma, but patients exhibited a higher incidence of minor hemorrhagic events: blood aspirated through spinal or epidural needle or catheter.

In a follow-up prospective study of 1000 patients undergoing orthopedic procedures with neuraxial anesthesia, Horlocker et al (224) demonstrated that 
pre-operative antiplatelet therapy had no correlation with the presence of blood at the needle hub or during catheter placement or removal. These results differed from their retrospective study, but once reporting of minor hemorrhagic events was standardized, the rate increased from $2 \%$ (223) to $22 \%$ (224). Despite asserting that the risk of spinal hematoma is not significant in patients receiving antiplatelet therapy and neuraxial anesthesia, Horlocker et al (224) surmises that there may be a correlation between minor spinal canal bleeding and spinal hematomas: these hematomas represent clinically insignificant collections of blood in the spinal canal. This implies that pre-operative antiplatelet therapy is not a significant risk factor for the development of neurological dysfunction from spinal hematoma. A world famous hematologist, however, questions whether these data are sufficient to exclude the possibility of a hematoma in a patient that consumes aspirin and receives a neuraxial anesthetic (225).

Subarachnoid hematomas following lumbar puncture can occur in the presence of anti-platelet therapy, along with other causes of reduced platelet function or numbers $(184,220,226-228)$. Erythrocyte counts were not significantly increased during subarachnoid injections, in patients receiving antiplatelet therapy. Knowles et al (229), however, cautioned that the risks associated with lumbar puncture and antiplatelet therapy are of prolonged bleeding, rather than the initial vascular injury (229). In fact, delayed hematomas have occured (228), in the presence of anti-platelet therapy.

Aspirin is not a contraindication to non-neuraxis blocks, despite concerns among clinicians $(26,220)$. Acetylsalicylic acid administration, following femoral nerve blocks, did not result in more hematomas (230). Aspirin use is not associated with an increased risk of retrobulbar hemorrhage and should not be discontinued prior to retrobulbar blocks (196). However, Sub-Tenon anesthesia is associated with an increased incidence of minor subconjunctival hemorrhagic in patients consuming aspirin compared to those not taking aspirin. Surgery could not proceed in $4 \%$ of these aspirin-takers (231). Spontaneous orbital hemorrhage has been reported in a patient that was consuming fenoprofen and aspirin (232).

\section{Thienopyridine Derivatives and} Glycoprotein Receptor Antagonists

The risk of bleeding, during regional blocks, may be increased with thienopyridine derivatives relative to aspirin and NSAID therapy (209). Thienopyridine agents have been implicated in two cases of severe bleeding following lumbar sympathetic blocks (233). The authors followed a strict and safe approach, with fluoroscopic control, aspiration, small caliber sharp needles, and contrast. Ticlopidine was not held prior to the block in one patient and the hematocrit dropped. Nonetheless, a second block was performed 6 days later. Several hours later, the patient complained of groin pain and developed hypotension. There was a further reduction in the hematocrit and a retroperitoneal hematoma was diagnosed. The patient was transfused. In the second case, a lumbar sympathetic block was performed three days after discontinuing clopidogrel. Coagulation studies, including bleeding time, were normal. Several hours later, the patient developed groin pain and suffered a cardiac arrest. An autopsy demonstrated a large retroperitoneal hematoma (233). This complication unfavorably compares to the $<0.1 \%$ incidence of retroperitoneal hematomas, following lumbar plexus blocks in general (233). Ideally, ticlopidine should be held for 10-14 days and clopidogrel for 7 days (5). Some suggest holding both medications for only 7 days $(233,234)$. Further caution is advised if these agents are used in conjunction with aspirin or NSAIDs.

A few spinal hematomas that developed following a neuraxial procedure, but were attributed to thienopyridine derivative therapy, have been reported $(227,234$, 235). The synergy between several antiplatelet agents may significantly increase the risk of a hematoma. A patient developed a cervical epidural hematoma following a cervical epidural steroid injection. He was consuming diclofenac, aspirin, and clopidogrel at the time the procedure was performed (234).

Due to the variety of pharmacological actions of antiplatelet agents, predicting the risk of a spinal hematoma following a neuraxial block is impossible (5). A history of easy bruisability, female gender, and increased age may increase the risk of a hematoma with antiplatelet agent (5). Aspirin and NSAIDs do not pose a significant bleeding risk in performing neuraxial procedures and there are no issues re- garding the timing of the block (5). Since the risk associated with thienopyridine derivative and glycoprotein receptor antagonists is unknown, the American Society of Regional Anesthesia has adapted guidelines from the radiology and cardiology literature for neuraxial procedures: withhold ticlopidine for 14 days and clopidogrel for 7 days and avoid procedures 4 weeks after $\mathrm{Gp}$ receptor antagonists. At a minimum, platelet function should normalize following $\mathrm{Gp}$ receptor antagonist administration: 24-48 hours for abciximab and 4-8 hours for eptifibatide and tirofiban (5). If a patient consumes several antiplatelet drugs, synergistic actions may have a profound effect on platelet function; thus, these agents may have to be held for 5-7 days (234).

\section{DeXtran}

Only one epidural hematoma, following a neuraxial procedure has been reported in a patient receiving dextran therapy (111).

\section{WARFARIN}

Oral anticoagulation with warfarin is a contraindication to neuraxial anesthesia (5). Atraumatic epidural catheterization was performed in one patient that was fully anticoagulated with warfarin; the anesthesiologist was unaware and the patient developed paraparesis (115).

Neuraxial anesthesia in patients who have discontinued coumadin or will be receiving coumadin for thromboprophylaxis is more controversial. Survey data from 1998 regarding opinions of anesthesiology program directors in the United States on perioperative discontinuance or alteration of medications, specifically warfarin, have been reported. The length of time physicians would discontinue warfarin therapy, prior to surgery, is variable. However, $72 \%$ of the respondents favored the use of a heparin window preoperatively. Further data may be needed to assess which approach is more appropriate for the use of anticoagulants in operative candidates (236).

No hematomas have been reported in patients receiving perioperative warfarin for thromboprophylaxis (5). Odoom and Sih (237) performed 1000 epidurals in 950 patients undergoing vascular surgery. All the patients were taking oral anticoagulants pre-operatively and received heparin intra-operatively. A pre-operative thrombo-test (to assess factor IX activity) was decreased and a post-operative aPTT 
was elevated. Catheters were left in place for 48 hours. No hematomas developed.

A few studies have investigated the safety of perioperative, low dose warfarin prophylaxis and epidural anesthesia. $\mathrm{Wu}$ et al (238) removed epidural catheters an average of 43.6 hours after placement for hip and knee surgery; low dose warfarin prophylaxis was used and the average prothrombin time increased from 10.8 (9.611.1 , normal range) to 14.1 seconds at removal. In another series (239), one hundred eighty-eight patients received warfarin prophylaxis following a total knee replacement. Epidural catheters were placed for post-operative pain control. They were left indwelling an average of 37.5 hours and the mean PT during catheter removal was 13.4 (range: 10.8-12.8). The PT did not exceed the upper range of normal until the third post-operative day and did not reach 15 seconds until the $7^{\text {th }}$ postoperative day. There were no epidural hematomas in either series. Due to the variability in warfarin response, monitoring the PT is advised prior to catheter removal $(5,238,239)$. In one case report, a single pre-operative dose of warfarin, $10 \mathrm{mg}$, increased the INR to 6.3 by the second postoperative day. The catheter was nonetheless removed. The patient developed paraparesis secondary to an epidural hematoma and subsequently, required emergent decompression (240).

Badenhorst (241) reported an epidural hematoma in a patient who had a catheter removed with a PT of 17.3 seconds (normal range:11.2-14.4 seconds). Several other cases through the MedWatch system have been reported. In one case the PT was 50 seconds at the time of needle placement and another in which the INR was 1.6 at the time the neurological deficit was diagnosed and the catheter was indwelling. Wu, et. al., however, reports that $20 \%$ of patients in his series had epidurals removed when the PT was greater than 16 seconds $(205,238)$.

The American Society of Regional Anesthesia suggests that warfarin should ideally be stopped 4-5 days prior to neuraxial procedures. The prothrombin time and international normalized ratio should be checked the prior to the neuraxial block. An early reduction in the PT/ INR reflects replenishment of factor VII: the remaining Vitamin K-dependent factors take longer to normalize and consequently, so does return of normal hemostasis. The effect of warfarin may be en- hanced when antiplatelet agents, fractionated heparins, and unfractionated heparins are co-administered $(46,242)$, without affecting the PT/INR. Hence, despite advocates for off-label, outpatient 'bridging therapy' with low molecular weight heparin (119), bleeding risk may actually be increased. There is no 'safe' PT/INR for performing neuraxial procedures, despite literature suggesting that an INR of 1.5 (120) is safe for major surgical procedures. In one institution, an INR of $<1.3$ is safe for neuraxial procedures and an INR $>1.5$ is unsafe; if the INR falls between 1.3 and 1.5 , a neuraxial procedure may proceed in the absence of other bleeding disorders or anticoagulants (119).

If low dose warfarin is administered in the presence of an indwelling epidural catheter, the PT/INR must be monitored daily. Catheter removal may be performed with an INR $<1.5$. European guidelines suggest that therapy should be stopped for 48 hours and the INR should be $\leq$ 1.4 (20). Serial neurological examinations should be performed for a minimum of 24 hours following catheter removal or longer in the case of higher warfarin doses (5). If the INR $>3$ in the presence of an indwelling catheter, then warfarin should be held or reduced in dose (5). No definitive recommendations are given for the removal of a catheter during therapeutic anticoagulation (5), however, an INR $>3$ may warrant a reduction of dose or cessation of therapy (243). Due prudence is required in this situation.

Very little information is available on the performance of non-neuraxial blocks in the setting of oral anticoagulation. Kallio et al (196) prospectively graded the hemorrhages following retrobulbar/peribulbar block. They concluded that the pre-operative use of warfarin, ASA, NSAIDs, whether discontinued or not, did not pre-dispose to hemorrhage in these blocks (196). However, they advised holding warfarin for two days before peribulbar blockade. Maxillary and mandibular nerve blocks are contraindicated in the setting of oral anticoagulation: vessel puncture presents a high risk of hematoma formation (244). Sublingual or submaxillary hematomas have never been reported following facial blocks, but such a complication could cause severe airway compromise (245). Similar concerns may apply to other blocks in the head. Distal extremity blocks can result in complications. Parziale et al (118) report- ed the development of compartment syndrome following a median nerve injection in a patient receiving warfarin. It is not known, if and to what extent, the guidelines for neuraxial anesthesia apply to interventional pain procedures.

Nonetheless, if an expansile hematoma occurs due to needle injury, in a patient that is anticoagulated with coumadin, urgent care is warranted. Fresh frozen plasma and vitamin $\mathrm{K}$ are used to reverse anticoagulation $(114,118)$. Close monitoring of vital signs, prothrombin time, and hematocrit are imperative and surgical attention may be necessary (118).

\section{Thrombolytics And Fibrinolytics}

Although no trials have investigated the safety of neuraxial anesthesia in the presence of thrombolysis or fibrinolysis (205), thrombolytics are an absolute contraindication to regional anesthesia (5). There are several reports of spontaneous spinal hematoma following thrombolysis $(129,246-250)$, but few in association with neuraxial techniques (251-253). In most of these cases, thrombolytics were administered perioperatively, with or with out heparinization. Significant bleeding reportedly may present anywhere from a few hours to a few days after a neuraxial procedure.

If thrombolysis is anticipated, patients should be advised against neuraxial blocks (5). There are no data are available for how long neuraxial procedures should be held after thrombolytics have been administered (5). The action of thrombolytic drugs may take days to resolve, due to the presence of fibrin degradation products.

The ASRA guidelines are very firm and cautionary in avoiding neuraxial techniques during the administration of thrombolysis/fibrinolysis: 1. Concurrent heparin use with thrombolytics/fibrinolytics exposes patients to a high risk of adverse spinal bleeding following neuraxial procedures; 2. Neuraxial techniques should be avoided in these patients, unless highly unusual circumstances are present; 3. Patients and their clinicians should be queried pre-operatively to determine if thrombolytics/fibrinolytics had been used pre-operatively or if these agents will be used perioperatively; 4 . If thrombolytics are given around the time of the neuraxial block, serial neurological monitoring should be performed at an interval of 2 hrs and the drugs used in neuraxial infu- 
sions should be minimized to avoid sensory-motor blockade; 5 . No recommendations were provided about neuraxial catheter removal or maintenance in those patients who unexpectedly receive fibrinolytic or thrombolytic therapy (129). Fibrinogen levels may be helpful in clinical decision-making (129).

\section{HEPARIN}

Vandermeulen (208) reported 30 cases of epidural hematoma in patients that received heparin therapy in a variety of forms: unfractionated or low molecular weight, subcutaneous or intravenous, single or multiple boluses, or continuous. Heparin therapy was responsible for the majority of hematomas associated with abnormal hemostasis (208). Due to the risk of epidural hematoma, $70-75 \%$ of neuraxial blocks in Europe are single dose spinal anesthetics and the majority of neuraxial procedures are performed on in-patients that received heparin thromboprophylaxis (unfractionated or low molecular weight) the day before $(5,254$, 255). American, German, and Spanish (3, $4,5)$ guidelines have been published with respect to neuraxial anesthesia and heparin therapy. These guidelines are similar with respect to unfractionated heparin (3, $4,5)$, but American guidelines are more conservative with respect to LMWH (5).

\section{Subcutaneous Heparin}

In 1981, Stanton-Hicks (256) suggested that neuraxial anesthesia is contraindicated in the presence of heparin thromboprophylaxis. There have been a few cases of epidural hematoma in association with low dose heparin, following an epidural (257, 258). Subcutaneous heparin therapy was considered an absolute or relative contraindication to epidural and spinal anesthesia in $38 \%$ and $24 \%$, respectively of Danish anesthesiology departments (115). Opinions have since changed. Tryba et al (254) reported that mini-dose heparin is not a contraindication to spinal or epidural anesthesia. Currently, neuraxial anesthesia is considered to be safe in the setting of subcutaneous, mini-dose heparin $(141,142)$, as suggested by the absence of complications in more than 9000 patients (133). Most anesthesiologists in Europe do not consider subcutaneous heparin to be a strong contraindication to neuraxial blockade $(5,115,259)$.

Spinal hematomas have been reported in association with neuraxial tech- niques and subcutaneous heparin (208, 260-262), but many of these cases had complicating factors, such as traumatic needle placement. Ideally, a neuraxial block should be performed prior to or after the injection of subcutaneous heparin, but there does not appear to be an increased risk when performed in the presence of subcutaneous heparin $(5,133)$. Delaying the performance of a neuraxial block for 2 hours after subcutaneous heparin may coincide with the peak effect and is not advised (5). Risk may be increased in debilitated patients on prolonged doses of subcutaneous heparin. Patients on subcutaneous heparin for greater than 4 days should be checked for a platelet count (5). Otherwise, there are no contraindications to neuraxial anesthesia in the presence of subcutaneous heparin.

\section{Systemic Heparinization}

Systemic heparinization, following neuraxial anesthesia, has (199) and has not $(237,263)$ resulted in adverse bleeding sequelae. Ruff et al (264) reported the complications in 2 groups of 342 patients that underwent lumbar puncture. One group received systemic heparinization and the other group did not. There were 7 spinal hematomas, of which 5 patients developed paraparesis, in the anticoagulated group. Risk factors included administration of heparin within 1 hour after lumbar puncture, traumatic needle placement, and concomitant use of aspirin (264).

In a retrospective review, 912 patients underwent vascular surgery with epidural anesthesia. Patients received an intra-operative bolus of $75 \mathrm{U} / \mathrm{kg}$ of heparin, followed by a $1000 \mathrm{U} /$ hour infusion. Intra-operative aPTT was documented at one point to be $>100$ seconds. At the end of the case, epidurals were removed without checking an aPTT. Surprisingly, no patient developed adverse neurological problems (265).

In a recent prospective study of 305 patients undergoing valvular surgery, thoracic epidurals (TEA) were placed at T13 and strict guidelines were followed. Preoperative selection criteria for TEA included an activated partial thromboplastin time of $<45$ seconds, prothrombin levels $>50 \%$, platelets $>80,000$, and no antiplatelet agents for 7 days, prior to the procedure. Systemic heparinization was held for at least $60 \mathrm{~min}$ utes after thoracic epidural placement and an atraumatic needle insertion was mandated. Catheters were removed within 48 hours or at any time, if patients were started on coumadin or aspirin, respectively. There were no epidural hematomas (266).

Similar guidelines were followed in a series of 508 patients undergoing coronary bypass surgery and no epidural hematomas developed (267). There have been no reported spinal hematomas in over 5000 patients undergoing cardiac surgery, with many series following guidelines as strict as those above $(266,268)$. Further recommendations include use of a midline technique, instillation of saline to distend the epidural space prior to catheter insertion, and catheter placement 24 hours prior to surgery $(5,267,268)$. Significant breach of these recommendations may increase the risk of an epidural hematoma, during cardiac surgery (268).

Ho et al (268) estimates the risk of a spinal hematoma in conventional cardiac surgery, using a $95 \%$ confidence level ranges between 1:150,000 to 1:1500 for epidurals and 1:220,000 to 1:3600 for spinals. If a more stringent, 99\%, level of confidence, is used then the upper range will be 1:1000 and 1:2400 for epidurals and spinals, respectively. The rationale for estimating the risk was to weigh the benefits of epidural anesthesia in reducing the mortality of cardiac surgery and myocardial infarction, against the risks of an epidural hematoma (268). Nonetheless, ASRA suggests that there is insufficient data about neuraxial anesthesia in the setting of cardiac surgery (5).

The safety of neuraxial anesthesia in the setting of systemic heparinization is controversial. Some authorities do not consider it safe (145) and others do (133, 263). In order to maximize the safety, Rao et al (263) advises canceling the case if traumatic needle insertion occurs, selecting patients carefully, and monitoring anticoagulation carefully $(208,263)$.

Stafford-Smith (201) calculated a significantly increased relative risk of epidural hematoma with traumatic needle insertion in the presence of intravenous heparinization. The extensive and published clinical experiences of numerous centers attest that regional techniques are safe, in the presence of therapeutic, intraoperative heparinization (133).

In the setting of cardiac surgery, several guidelines for neuraxial anesthesia have been published: 1 . Neuraxial blocks should be avoided in patients with known coagulopathy from any cause. 2 . Surgery should be delayed for 24 hours if there is 
a traumatic tap. 3. $>60$ minutes should elapse between the neuraxial block and initiation of systemic heparinization. 4 . Heparin effect and reversal should be tightly controlled. 5. Epidural catheters should be removed when normal coagulation is restored. 6. patients should be monitored closely for neurological deficits (269). Many case series have followed similar guidelines with safe outcomes (5, 133).

In the setting of vascular surgery, neuraxial anesthesia appears to be safe with certain guidelines: 1 . Avoid the technique in patients with other coagulopathies; 2. Heparin administration should be delayed for 1 hour after needle placement; 3. Indwelling neuraxial catheters should be removed 2-4 hours after the last heparin dose, after coagulation status is assessed; 4. Re-heparinization should be delayed for 1 hour after catheter removal; 5. Post-operative neurological monitoring is mandated and the minimal concentration of local anesthetics that affords pain relief, but does not mask any neurological deficit, should be used; 6 . Traumatic or difficult needle insertion may increase the risk of a hematoma, but case cancellation may not be mandatory (5). The issue of case cancellation when a traumatic puncture occurs is disputed $(206,207,261)$.

Therapeutic anticoagulation for arterial thrombotic disease usually requires an elevation of aPTT to 1.5-2 times normal. In this situation, the risk of spinal hematoma may outweigh the benefits and neuraxial techniques should be avoided in this population (133). If therapeutic anticoagulation, however, is started in the presence of an indwelling catheter, then the catheter should be removed 2-4 hours after discontinuing heparin and after checking an aPTT (133).

Peripheral nerve blocks have emerged as an alternative to neuraxial blockade, when aggressive anticoagulation is anticipated (1). The enhanced safety of non-neuraxial over neuraxial procedures, during systemic heparinization, is surmised since the tissue is more compliant and less likely to cause neural compression (1). The hazard of this assumption is that diagnosis can be delayed (1). In fact, non-neuraxial procedures have resulted in significant bleeding following systemic heparinization. An intercostal catheter was placed 2 hours following a subcutaneous heparin injection. Coronary bypass surgery was subsequently performed with intra-operative heparinization. Despite the complete reversal of anticoagulation at 6 hours post-operatively, a flank hematoma developed within the following 24 hours (270). Therapeutic intravenous heparinization has been implicated in another flank hematoma, following intercostal nerve blocks (271). A delayed retroperitoneal hemorrhage can occur, following the placement of a lumbar plexus block or catheter, wherein systemic anti-coagulation is performed (1). A hemothorax developed following a supraclavicular brachial plexus block, even though systemic heparinization was commenced greater than 4 hours post-procedure (272). The paucity of data on nonneuraxis procedures make it difficult to provide recommendations (1), but systemic heparinization is considered a contraindication (271).

\section{Low Molecular Weight Heparin}

In a two separate reviews of published series, more than 23,000 patients in Europe received LMWH and neuraxial anesthesia without any hematomas (141, 142, 273). In Europe, the regimen was once-daily dosing and an initial dose was given at least 12 hours before surgery. The initially approved regimen for thromboprophylaxis in the USA was twice daily dosing and an initial dose given immediately after surgery. Twice daily versus single daily dosing resulted in a significantly greater anticoagulant effect (274). Over a 5-year period, 1993-1998, sixty spinal hematomas were reported (5). Thirty of these were reported to the Food and Drug Administration MedWatch Program and an advisory warning was sent out (200). Thirteen hematomas were reported in Europe between 1989 and 1998 (5, 203). Adoption of practice guidelines in Europe may have reduced the frequency of spinal hematoma $(5,141,142)$.

Several dosing regimen changes have been implemented in the USA, since 1993. LMWH should be started 12-24 hours after surgery and once daily dosing of enoxaparin, $40 \mathrm{mg}$, may be sufficient for DVT prophylaxis. Nonetheless, 30mg SC BID is still commonly used with the first dose given post-operatively. Recently, the LMWH, dalteparin has been approved for use with a regimen that more closely mimics the European guidelines: half the standard dose, 2500 Units, is administered 6-8 hours after surgery and a standard dose, 5000 Units is given 24 hours later
(5). Once-daily dosing may be safer for continuous indwelling catheters (5).

The majority of cases of spinal hematoma, associated with low molecular weight heparin, occurred in the elderly and in women $(202,203)$. For instance, a thoracic epidural hematoma developed, wherein an epidural catheter was removed just 2 hours after LMWH administration (275). Clinical signs of neurological dysfunction, due to a spinal hematoma, typically present 3 days after LMWH therapy. The elapsed time between symptom onset and laminectomy was 24 hours (5). Close neurological and hemodynamic monitoring, serial hematocrit measurements, and inspection for other bleeding sites are advised for non-neuraxis and neuraxis procedures (26). In 1998, the estimated risk of spinal hematoma in the setting of LMWH, following neuraxial anesthesia was 1:3,000 for continuous epidural anesthetics and 1:40,000 with spinal anesthetics (276).

Despite confounding variables, such as vascular disease, diabetes, advanced age and concomitant aspirin usage, low molecular weight heparins are considered to increase the risk of bleeding complications following a regional technique (145). Notably, this contrasts with the surgical literature. In a recent meta-analysis, low molecular weight heparins do not increase the risk of bleeding compared to placebo, in orthopedic surgery (277).

Retroperitoneal hematomas have developed when lumbar plexus blocks were performed in concert with perioperative administration of LMWH $(1,145)$. Two patients $(1,145)$ developed a psoas hematoma following a lumbar plexus block and perioperative use of low molecular weight heparin. In one patient, low molecular weight heparin was administered 40 hours after the block, but the catheter was removed only 2 hours after this dose (1). The patient developed severe flank pain, but no neurological deficit. CT-scanning confirmed the diagnosis and the patient received a blood transfusion. In another patient, the lumbar plexus block was performed 20 hours after the last dose of LMWH, but with technical difficulties. On the first post-operative day, the patient complained of hip pain and hip flexor weakness. A CT-scan demonstrated a large retroperitoneal hematoma. This patient was also managed conservatively and made a good neurological recovery (145). Spontaneous retroperito- 
neal hematomas may also develop following enoxaparin use, particularly in the elderly $(138,278)$.

The diagnosis of a retroperitoneal hematoma depends on the presence of flank, groin, and medial thigh pain and hip flexor weakness $(1,233)$. Confirmation of the diagnosis is made with emergent radiological studies $(1,145,233)$. The retroperitoneal space is not confined, so morbidity is typically due to the degree of blood loss, rather than actual neurological dysfunction (5). In severe cases, patients may succumb (233). However, conservative management is often successful: serial neurological, hematological, and hemodynamic monitoring, reversal of anticoagulation, and possibly, blood transfusions $(1,145)$.

In 1998, the American Society of Regional Anesthesia published guidelines for neuraxial anesthesia in the setting of LMWH (210). Since these guidelines were published, epidural hematomas associated with LMWH have occurred 6 times, spontaneously and 13 times following neuraxial procedures. Ten of these 13 were either from outside the USA or were receiving ketorolac (5). One patient received ibuprofen and one received intravenous unfractionated heparin during a vascular procedure (5).

The recommendations for the perioperative use of low molecular weight heparin and neuraxial anesthesia were recently updated by the American Society of Regional Anesthesia and Pain Medicine (5): 1. Anti-Xa levels do not have to be monitored, since they do not predict bleeding risk; 2 . concomitant use of antiplatelet agents or oral anticoagulants should be avoided; 3 . traumatic needle or catheter insertions, i.e., blood is aspirated or spontaneously appears, may significantly increase the risk of spinal hematoma; 4. traumatic neuraxial procedures are not mandatory grounds for case cancellation, but LMWH administration should be delayed for 24 hours.

Recommendations for the pre-operative administration of LMWH include: 1 . If prophylactic doses are given, then needle placement be delayed for at least 10-12 since the last dose; 2 . If therapeutic doses are given, then needle placement must be delayed at least 24 hours since the last dose. Therapeutic doses of low molecular weight heparin include enoxaparin $1 \mathrm{mg} /$ $\mathrm{kg}$ q12 hours or $1.5 \mathrm{mg} / \mathrm{kg} \mathrm{qD}$, dalteparin $120 \mathrm{U} / \mathrm{kg}$ q12 hours or $200 \mathrm{U} / \mathrm{kg} \mathrm{qD}$, and tinzaparin $175 \mathrm{U} / \mathrm{kg}$ qD. Under no circumstances should a neuraxial technique be performed if the last dose of LMWH was given only 2 hours earlier.

Single shot neuraxial or continuous epidural techniques can be performed with the post-operative administration of $\mathrm{LMWH}$, however, the recommendations for this situation are more complex. If post-operative LMWH is to be given twice-daily, then LMWH should be held for at least 24 hours after surgery and indwelling catheters should be removed prior to LMWH initiation; this implies that indwelling catheters may be left overnight, but should be removed at least two hours before the first dose of LMWH.

Post-operative LMWH may also be given once-daily, which mimics European prescribing practices. In this situation, the first dose should be administered 6-8 hours after surgery and the second dose must be given at least 24 hours after the first dose. An epidural catheter can be safely maintained under these circumstances. If the catheter is to be removed, there should be a minimum time interval of 10 to 12 hours since the last dose of LMWH. LMWH can then be started a minimum of two hours after catheter removal (5).

Similar guidelines have been advocated by other authors for neuraxial and non-neuraxial procedures $(1,145,208)$. Vandermeulen et al (208) advocated a 1012 hour low molecular weight heparin free interval before the removal of needles or catheters, due to the pharmacokinetics of these agents (208). Gerancher et al (1) recommends that lumbar plexus blocks or catheters be placed at least 12 hours since the last dose of prophylactic LMWH. Remove or do not place catheters if therapeutic anticoagulation is planned. LMWH can be re-started 2 hours after the catheter is removed. One caveat is that hospital staffs must administer LMWH at a set time and follow protocols (279). Even then, nursing compliance can be poor and thus, physicians must verify the timing of administration (279). Compliance issues will play a greater role as outpatient LMWH therapy gains wider acceptance. Finally, whether these guidelines are applicable to interventional pain management is unknown.

\section{Multiple agents}

The use of multiple anticoagulants increases the risk of significant bleeding, especially if their actions are synergistic. Intra-operative bleeding, from a continuous spinal, has caused severe hypotension. The patient had diabetes and peripheral vascular disease and was receiving aspirin therapy. There was difficulty with needle insertion during the spinal, but the procedure was finally successful. An 18 gauge needle was used to pass a 20 gauge bullet tipped catheter into the subarachnoid space. Intra-operatively, the patient received heparin, but a hypotensive episode preceded this. Almost a liter of blood was lost due to a subcutaneous bleeder located around the catheter site. The authors concluded a multifactorial etiology to bleeding: traumatic injury to subcutaneous vessel, aspirin, intra-operative heparin, difficulty with needle insertion; fortunately no neurological compromise developed (26).

Aspirin, NSAIDs, and COX-2 inhibitors alone may not increase hematoma risk, but when used together or in concert with other anticoagulants, the risk of hematoma and minor bleeding may increase $(138,210,234,264)$. COX-2 inhibitors can augment the effects of coumadin (5). An epidural hematoma occurred in a patient that received aspirin and dipyridamole, followed by systemic heparinization; the heparin was held for 3 hours before and 2 hours after catheter removal (280). High dose ibuprofen consumption and perioperative administration of low molecular weight heparin have been implicated in the development of an epidural hematoma following epidural catheterization (281).

Surgical bleeding can become worse in the presence of mini-dose heparin and dextran and the combination should be avoided $(67,282)$. This scenario may apply to percutaneous interventions, as well.

One patient had normal coagulation studies during performance of a lumbar plexus block. Systemic heparinization and oral coumadin therapy were initiated eight hours later. On the third post-operative day, the patient complained of flank and groin pain. The PT, PTT, and hematocrit were abnormal. A CT-scan confirmed the presence of a large psoas hematoma (1). Other studies of peripheral nerve blockade with multiple anticoagulants have not confirmed this. Singelyn et al ( 283) in a prospective series of patients receiving extended femoral sheath blocks for total hip arthroplasty did not identify any neurologi- 
cal or vascular complications; all patients received low-molecular weight heparin and an antiplatelet agent daily, starting the night of surgery and ketorolac every 8 hours as needed was given (283).

\section{HeRbal MEDiCATIONS}

A spontaneous epidural hematoma has occurred following garlic consumption (284). The American Society of Anesthesiology recommends discontinuing herbal medicines for 2-3 weeks prior to elective surgery (285). Herbal medications, such as garlic, ginseng, ginger, and ginkgo may be a risk factor for spinal bleeding associated with neuraxial anesthesia, especially if patients are consuming other anticoagulants (152).

\section{New Anticoagulants}

Several new anticoagulants have been developed. The safety of these agents, with respect to interventional pain practice, is unknown. Fondaparinux is not thought to increase the risk of epidural hematomas following a neuraxial procedure (286).

Study patients, however, do not represent typical clinic and hospital settings. In a study evaluating the efficacy of fondaparinux for venous thromboembolism prophylaxis for hip surgery, inclusion criteria were strict. All neuraxial procedures had to be done atraumatically and with one attempt. Indwelling catheters were removed 2 hours before the first dose of fondaparinux administration. These guidelines are not feasible in clinical practice $(5,163)$. Since the risk of an epidural hematoma with fondaparinux is unknown, a few tentative recommendations have been provided: continuous, indwelling regional methods should be avoided and the block should be atraumatic and successful with the first attempt (5).

\section{Procedure-Associated Bleeding Complications}

Technical difficulties have been implicated as a factor in major $(5,20,208)$ and minor (222-224) bleeding events, following neuraxial procedures. Technical factors may similarly be implicated in bleeding complications following interventional pain management procedures. The literature, with the exception of neuraxial procedures, is limited. Procedures can be roughly divided based on whether they are neuraxial or non-neuraxial. Additionally, the procedures can be organized topographically, based on body regions (Table 6). We have organized the available literature accordingly.

\section{Non-Neuraxial TeChNiques}

Perineural hemorrhage may be a particularly important factor when regional blocks require intentional vessel

Table 6. Topographic classification of commonly practiced interventional techniques for the treatment of acute and chronic pain.

\begin{tabular}{|c|c|c|c|c|}
\hline Head and Neck & $\begin{array}{l}\text { Chest, Upper Limbs, and } \\
\text { Thorax }\end{array}$ & Lumbar and Abdomen & Pelvis & Lower Extremities \\
\hline Trigeminal ganglion & Brachial Plexus & $\begin{array}{l}\text { Lumbar spinal nerve and } \\
\text { dorsal root ganglion }\end{array}$ & Sacral nerve root & $\begin{array}{l}\text { Sciatic nerve (infragluteal } \\
\text { and popliteal) }\end{array}$ \\
\hline Maxillary nerve & Radial Nerve & Splanchnic nerve & $\begin{array}{l}\text { Superior hypogastric } \\
\text { plexus }\end{array}$ & Piriformis \\
\hline Mandibular nerve & Median nerve & Celiac plexus & Sacroiliac joint & Femoral nerve \\
\hline Glossopharyngeal nerve & Ulnar nerve & $\begin{array}{l}\text { Lumbar sympathetic } \\
\text { ganglion }\end{array}$ & Ganglion impar & Obturator nerve \\
\hline $\begin{array}{l}\text { Cervical spinal nerve and } \\
\text { dorsal root ganglion }\left(\mathrm{C}_{2}-\mathrm{C}_{7}\right)\end{array}$ & Musculocutaneous nerve & $\begin{array}{l}\text { Lumbar facet and medial } \\
\text { branch block }\end{array}$ & Sacroplasty & Saphenous nerve \\
\hline Sphenopalatine ganglion & Intercostal nerve & $\begin{array}{l}\text { Lumbar provocative } \\
\text { discography }\end{array}$ & $\begin{array}{l}\text { Superior cluneal } \\
\text { nerves }\end{array}$ & Sural nerve \\
\hline Stellate ganglion & $\begin{array}{l}\text { Thoracic spinal nerve and } \\
\text { dorsal root ganglion }\end{array}$ & $\begin{array}{l}\text { Intradiscal procedures } \\
\text { (intradiscal electrothermal } \\
\text { therapy, radiofrequency } \\
\text { annuloplasty, nucleoplasty, } \\
\text { laser disc decompression, } \\
\text { percutaneous } \\
\text { decompression) }\end{array}$ & & Common peroneal \\
\hline $\begin{array}{l}\text { Cervical facet and medial } \\
\text { branch }\end{array}$ & Suprascapular nerve block & Vertebroplasty, Osteoplasty & & Posterior tibial \\
\hline $\begin{array}{l}\text { Cervical epidural space } \\
\text { including adhesiolysis }\end{array}$ & $\begin{array}{l}\text { Thoracic sympathetic } \\
\text { ganglion (upper and lower) }\end{array}$ & $\begin{array}{l}\text { Psoas, quadratus lumborum } \\
\text { muscles }\end{array}$ & & \\
\hline \multirow[t]{3}{*}{ Cervical discogram } & $\begin{array}{l}\text { Thoracic facet and medial } \\
\text { branch }\end{array}$ & $\begin{array}{l}\text { Lumbar epidural space } \\
\text { including adhesiolysis an } \\
\text { endoscopy }\end{array}$ & & \\
\hline & Thoracic discogram & Lumbar plexus & & \\
\hline & $\begin{array}{l}\text { Thoracic epidural } \\
\text { procedures including } \\
\text { adhesiolysis }\end{array}$ & $\begin{array}{l}\text { Ilioinguinal, iliohypogastric, } \\
\text { and genitofemoral }\end{array}$ & & \\
\hline
\end{tabular}

Adapted from: Raj PP, Lou L, Erdine S et al. Radiographic Imaging for Regional Anesthesia and Pain Management. Churchill Livingstone, Philadelphia, 2003. 
puncture or are performed in the presence of anticoagulation. In contrast, vascular complications after interventional cardiology procedures, which use a number of anticoagulants, are about $0.39 \%$. The size of the catheter and the degree of anticoagulation influenced the frequency of complications (287), but no neurological complications occurred. Furthermore, many patients demonstrate a delay of several hours, in the development of a hemorrhage or neurological dysfunction following a peripheral block and this has been used as an argument against blaming the technique $(233,288,289)$.

Controversy surrounds the mechanisms of neuropraxia following regional blocks. They may occur secondary to direct needle trauma, perineural hemorrhage, or local anesthetic toxicity (288292). Paresthesia elicitation, which implies direct neural contact, may result in a higher incidence of neurological sequelae (292).

Nonetheless, the following case reports demonstrate that there is still a risk of hemorrhage and secondary neuropraxia with non-neuraxis procedures. The actual rate may be higher than published data, even if safety measures are incorporated into the technique (293).

\section{Head and Neck}

Retrobulbar blocks may cause hemorrhage, typically venous, with an incidence ranging from $0.5-3 \%$ (294-296). Hemorrhages are more common in the elderly and in those with vascular disease (297). Independent of retrobulbar blockade, spontaneous hemorrhage of the orbit can still occur and has been associated with Valsalva maneuvers (labor, physical exertion, coughing during exertion), systemic autoimmune diseases, hypertension, and renal and vascular disease (232, 298). If these risk factors are present, alternative periocular injections should be considered. Hemorrhages are rarer with anterior orbital injections, when compared to retrobulbar blocks (295). Orbital hemorrhages are rarely of major consequence $(295,296)$. They can be managed with cold packs and observation. Aggressive intervention is needed if there is progressive blindness: a rising intraocular pressure may cause central retinal artery occlusion or optic nerve ischemia (295). These patients present with proptosis, a tightened orbit, opthalmoplegia, and peri-orbital blood staining (296). An oph- thalmology consultation is imperative for opthalmoscopy, intravenous mannitol, intravenous carbonic anhydrase inhibitors, lateral canthotomy, and perhaps, decompression and clot evacuation (294-296).

Hematomas or vascular injury following stellate ganglion blocks and cervical discograms are rare, but have been reported (299-301) in the absence of risk factors. In a survey of 39 departments of anesthesiology in West Germany, no bleeding complications were reported among over 45,000 stellate ganglion blocks (302). Intra-arterial puncture during a stellate ganglion block with a 20 gauge needle, complicated by seizures was reported in 2 cases (303). Post-operative sequelae were not reported (303). Others have confirmed the risk of intra-arterial puncture following stellate ganglion blocks $(304,305)$. In a series of 1357 patients undergoing cervical discograms, no hematomas were reported (306), but in another series of 269 cervical disc injections one patient developed a neck hematoma (300). Another cervical discography series with an overall higher rate of complications compared to those by Zeidman et al (306) and Guyer et al (300) also reported no hematomas related to discography (307). Hematomas following trigeminal and sphenopalatine ganglion procedures are rare. In a series of 496 patients undergoing 531 percutaneous trigeminal ganglion balloon decompressions, only 5 patients developed a facial hematoma (308). In an extensive review by Tew and Taha (309), in which over 8600 patients received percutaneous trigeminal ganglion procedures, there were no intracranial hematomas. Intracranial hemorrhage, however, has rarely been reported following these percutaneous procedures (310). In a series of sixty-six patients, sphenopalatine ganglion radiofrequency thermocoagulation has been associated with cheek hematomas and epistaxis in 8 and 11 patients, respectively (311). We could not identify any other reports of bleeding complications with non-neuraxial blocks in the head and neck region.

\section{Chest, Upper Limbs, and Thorax}

Brachial plexus blocks are rarely associated with major neurological or hematological complications. Axillary blocks are associated with $1-19 \%$ incidence of neural injury, but only $2-5 \%$ of patients have symptoms that persist beyond the immediate post-operative peri- od $(290,292,312)$. In a prospective study of 1000 patients, undergoing a standard transarterial axillary block with a 24 gauge needle, vascular and neurological complications were $1.4 \%$ and $0.7 \%$, respectively (312). In a perivascular, 'first indication of axillary sheath entry' approach and use of a 22 gauge short beveled needle, complications were common, but transient: $12 \%$ of patients had axillary bruising and tenderness and $12.5 \%$ of patients developed dysesthesias (313).

Urban et al (290) reported a neuropraxia rate of $9 \%$ in interscalene blocks and $19 \%$ in axillary blocks, within the first 24 hours. These rates dropped to $3 \%$ and $5 \%$, respectively, at 2 weeks. No hematomas were reported in these series but there was a $10 \%$ incidence of blood aspiration during the performance of an interscalene block (290). Eight percent of patients complained of neck bruising or inflammation on the first post-operative day after an interscalene block (290). There was no association, however, between aspiration and bruising (290). Overall, axillary nerve blocks had a higher rate of complications compared to interscalene block on the first post-operative day: $23 \%$ compared to $8 \%$ of patients complained of pain, tenderness, or bruising in the axilla or neck, respectively (290).

Transient symptoms such as paresthesias, dysesthesias, and pain not related to surgery may occur in $14 \%$ of patients, following an interscalene block or catheter placement (314). Infraclavicular blockade is associated with a $2 \%$ incidence of blood aspiration and a $0.6 \%$ incidence of hematoma formation (315). Seven percent of patients complain of pain following infraclavicular brachial plexus blockade (315). These types of symptoms are often considered minor by most anesthesiologists $(314,315)$.

In a series by Horlocker et al (288) six-hundred and seven patients underwent 1614 axillary blocks. Sixty-two neurological injuries were identified, but only 7 of these could be attributed to the anesthetic technique. Notably, several factors that are thought to increase the risk of anesthesia-related complications did not: age, gender, pre-existing neurological conditions, tourniquet time, surgical procedure on a nerve, and the performance of repeated blocks $(288,289)$. Similarly, no regional anesthetic technique factors-paresthesia elicitation, electrical stimulation, epinephrine, long-beveled needles- were 
identified as a cause of neurological injury (288). Two patients out of 607 developed an axillary hematoma, but suffered no adverse neurological sequelae. Repeat axillary blocks do not increase the risk of neurological complications compared to a single block (288).

An isolated case of a clinically significant hematoma following a brachial plexus block has been reported. An expanding, axillary sheath hematoma developed after an axillary nerve block and caused a radial nerve injury $(316,317)$.

Indwelling brachial plexus catheters may have rates of complications that are higher than blocks. Minor neurological symptoms occur at a rate of $2.4 \%$ following interscalene catheter placement, but drop to $0 \%$ at 6 months (318). However, complications related to technique occurred more often. In a series of 700 patients, 6 developed paresthesias and 12 had blood return during needle insertion. During catheter insertion, 25 patients developed transient shoulder pain, 13 developed dysesthesias, 42 patients presented anatomic resistance to catheter advancement, and 5 patients had blood return (318). In an earlier series, Borgeat et al (318) reported a $7.9 \%$ incidence of complications that dropped to $0.9 \%$ at 6 months, following a standard interscalene block (314). The authors credit their variation in interscalene block technique for a lower incidence of complications, compared to standard technique (318). Differences in brachial plexus block techniques may reduce the risk of technique-specific complications.

Neuropraxic symptoms, such as dysesthesias or paresthesias, may be due to perineural edema, inflammation, and microhematomas $(292,314,315)$. Paresthesias or electrical stimulation-induced elicitation may help localize neural structures for blocks, but do not protect against neural damage or perineural hemorrhage $(292,314,315,319)$. Despite the safety of peripheral nerve blocks, several have advocated avoiding these blocks in patients who need fine motor control, such as musicians (319).

Thoracic and lumbar paravertebral blocks are associated with a $6.8 \%$ incidence of inadvertent vascular puncture and $2.4 \%$ incidence of hematoma (320). Intercostal neural blockade, in the absence of hemostatic abnormalities, has rarely been reported as a cause of major bleeding: hemothorax (321). Hemotho- rax due to intercostal bleeding has been reported following attempted thoracic epidural cannulation by standard technique and thoracic paravertebral blocks (322-324). Thoracic discography has not been associated with vascular complications or hemorrhage in one large, recent study (325).

We could not identify any other reports of bleeding complications with nonneuraxial blocks in the thoracic and upper limb regions.

\section{Lumbar, Abdomen, Pelvis}

Paracervical injections have been associated with maternal demise, due to local anesthetic toxicity (326). Nonetheless, post-mortem studies have demonstrated the presence of blood near the broad ligament $(326,327)$. Blood can spread posterior to the broad ligament and even reach the sacrum (327). The absence of blood during aspiration might not exclude the possibility of intravascular penetration (326-328). Blind paracervical techniques have a large variation in the degree of needle depth penetration (327), so more superficial paracervical injections are advised to prevent vascular trauma (326, 327). A pudendal nerve block can injure the internal pudendal artery and lead to a retroperitoneal hematoma (328). Hematomas following pudendal and paracervical blocks can be clinically significant and cause neural injury, such as sacral neuritis $(327,328)$.

Vaisman (329) reported a pelvic hematoma following an ilioinguinal nerve block, with a 22-gauge sharp needle. Aspiration during the procedure was negative (329). Fluoroscopy or ultrasonography may help in safely and correctly placing an indwelling inguinal catheter (330). These methods may reduce the need for multiple punctures and the risk of vascular penetration $(329,330)$. A subfascial hematoma and compression syndrome has developed following a femoral nerve block in the absence of hemostatic abnormalities (331).

Hematoma formation following a lumbar plexus or lumbar sympathetic block is exceedingly rare. A percutaneous renal biopsy typically causes microscopic hematuria and only rarely, a major hematoma $(332,333)$. Renal subcapsular hematomas, following a lumbar plexus (334) and lumbar sympathetic (335, 336) blocks, have been reported. Aida et al (334) used a blind approach at L3 rath- er than L4, during their performance of a lumbar plexus block and did not comment on aspiration nor distance from midline (334). Despite use of conservative lateral (336) ( $5 \mathrm{~cm}$ from midline) or a far lateral (335) (12.5 cm from midline), renal subcapsular hematomas have been reported following lumbar sympathetic blocks. If the hematoma is larger, a Page kidney may occur and result in severe hypertension (336). Conservative management is possible with small hematomas, but a partial or complete nephrectomy may be required for large hematomas (336). Improvements in technique may reduce the risk of a renal capsular hematoma: 1. Lumbar plexus blocks should be performed at L4 or L5; 2. The transverse process must be encountered; and 3. fluoroscopy may be helpful (334). Retroperitoneal hemorrhages have been reported following a psoas compartment $(1,145)$ and lumbar sympathetic blocks (233), but in all these patients, anticoagulation was implicated.

We could not identify any other hemorrhagic complications related to non-neuraxial blocks. Refinements in technique and imaging, for instance, have reduced the risk of vascular injury or hemorrhage to almost nil in lumbar discography (337). Similar reasons may apply to other blocks in the lumbar, abdominal, and pelvic regions.

\section{Lower Limbs}

Several studies and reviews of lower extremity neural blockade (lumbar plexus blocks via fascia iliaca and femoral nerve sheath approaches, sciatic nerve blocks via posterior popliteal, lateral popliteal, gluteal, and subgluteal approaches) have demonstrated an absence of vascular or neurological complications (283, $338-341$ ). In a prospective series of 100 patients, undergoing a continuous 3 in 1 block, 2 patients developed a hematoma and 2 patients developed paresthesias extending to the quadriceps (342). There were no reported vascular complications in a series of patients that underwent peripheral nerve stimulation for complex lower extremity neuropathic pain (343).

\section{Neuraxial and Spinal Techniques}

Neuraxial and spinal techniques include epidural, subdural, subarachnoid, and selective spinal injections. There is a large body of literature investigating bleeding risk and complications in neur- 
axial anesthesia. One unique aspect about neuraxial anesthetics is that they have several effects on the coagulation cascade. Initially, the needle stick trauma may induce a stress response and activate the coagulation cascade. After the regional block (epidural or cervical plexus) is in place, the stress response to surgery may be blunted. Thus the activation of the coagulation cascade may be reduced (344). This may be partly responsible for the reduced risk of venous thromboembolism in patients receiving neuraxial anesthesia for lower extremity orthopedic procedures $(345,346)$. Fibrinolytic activity may be relatively preserved and clotting tendency may be reduced in epidural versus general anesthesia $(347,348)$. Others contend that neuraxial anesthesia has no effect on platelet function or fibrinolytic activity (345). Rather the reduced risk of venous thromboembolism is due to increased blood flow to the lower extremities secondary to the sympatholytic effects of neuraxial anesthesia $(345,346)$. It is not known if neuraxial anesthetics can themselves increase the risk of a spinal hematoma by affecting hemostasis.

\section{Spinal Hematoma: Diagnosis and Management}

Bleeding is classified as major if it is intracranial, intraspinal, intraocular, mediastinal, retroperitoneal, or if the bleeding results in death, hospitalization, or transfusion (5). Spinal canal hemorrhages most commonly occur in the epidural space, due to a prominent epidural venous plexus $(5,203,249,251)$. Spinal subarachnoid or subdural bleeding may be due to trauma of radicular vessels (203, 349). Fortunately, major spinal bleeding is rare. However, minor bleeding secondary to epidural venous puncture may occur in 2-11 percent of patients receiving neuraxial anesthetics (350).

Spinal epidural hematomas occur more often than expected and many remain asymptomatic $(185,186)$. Thirteen to fifteen percent of patients may not have identifiable risk factors $(20,208)$. Spontaneous spinal hematomas can occur, in the absence of coagulopathy (250). Others contend that spontaneous Spontaneous spinal hematomas typically occur in the presence of coagulopathy: anticoagulants, vascular malformations, chronic compression of epidural veins, and inherited or acquired bleeding disorders, such hemophilia and alcoholism (168, 252, 351-
353). In the absence of frank bleeding tendency, anticoagulation, or traumatic needle insertion, epidural hematomas have been reported following routine epidural catheterization (186).

One series estimated that coagulopathy is only involved in $26 \%$ of spontaneous spinal hematomas, but when present, the hematomas tend to be more extensive $(248,249)$. Abram et al (354) performed a literature review of complications associated with epidural steroid injections. Among 65 published series, with a total of 6,947 patients receiving one or more epidural steroid injections, there was only one case of a spinal hematoma $(218,354)$.

Symptoms depend on the location and the temporal qualities of the hematoma. Back pain and neurological dysfunction are the typical pre-monitory symptoms in lumbar and thoracic spinal hematomas $(189,208,226)$. Progressive sensorimotor loss occurs in $68 \%$ of patients (208) and bowel/bladder dysfunction in $8 \%$ of patients, but back and radicular pain are less common (208). In the cervical spine, patients have presented with Brown-Sequard Syndrome (356), cervical myelopathy, or flaccid tetraparesis (116, 357). Increased radicular/neck pain may be present $(116,356)$. The presentation can be immediate $(116,356)$, or delayed $(358,359,360)$. This delay can be as long as several days $(186,189)$.

The concentration of local anesthetic used for epidural infusion must be minimized in order to prevent masking of sensorimotor dysfunction. If there is no regression of neural blockade after stopping the infusion, one must have a high index of suspicion for an extradural hematoma (361).

Neuroimaging is imperative when these clinical signs are present. Magnetic resonance imaging is the most sensitive imaging modality for diagnosing an epidural hematoma, defining the extent of its spread, and distinguishing it from other space occupying lesions $(351,360)$. Epidural hematomas tend to be dorsally located $(116,362)$. The true incidence of epidural hematomas is unknown (360), but the number of reported cases has increased with the advent of magnetic resonance imaging (363).

Restoration of normal hemostasis, high dose corticosteroid therapy, and emergency decompressive surgery are advised for hematomas that cause neurolog- ical dysfunction $(356,359,360,364)$. If an epidural catheter is present, the infusion should be stopped and the catheter removed, in order to avoid increasing the compression (186). Aspiration is rarely successful due to the formation of a solid clot (351). These strategies have been adopted in numerous published cases with varying, but generally good outcomes.

$\mathrm{Ng}$ et al (360) replaced platelets in a patient with alcoholic cirrhosis and a platelet count of 67,000. Factor VIII can be used to treat spontaneous cervical epidural hematomas in patients with hemophilia $(166,359)$. Pullarkat et al (116) reversed anticoagulation with warfarin, using fresh frozen plasma and vitamin $\mathrm{K}$, in a patient that presented with a spontaneous onset of hematomyelia.

Groen et al (250) reported favorable outcomes in patients that underwent surgery for spinal epidural hematomas, depending on the time interval and degree of neurological dysfunction: 36 hours for complete sensorimotor loss and 48 hours for incomplete sensorimotor deficits (250). Patients with complete neurological loss and chronic compression may also benefit (364). Others contend that such delays are too long and that the critical interval is closer to 12 hours $(351,365)$. Foo et al (366) reports that the return of neural function following surgery for a spinal hematoma will depend on the severity of pre-operative neural deficits. Return of motor function was noted in $95.3 \%, 87 \%$, and $45.3 \%$ of the patients with incomplete sensorimotor, incomplete sensory but complete motor, and complete sensorimotor lesions, respectively. Complete sensorimotor recovery occurred in $41.9 \%$, $26.1 \%$, and $11.3 \%$ of these 3 groups of patients, respectively (366).

There are cases of spontaneous hematoma resolution $(186,367)$. A patient with hemophilia A developed a spontaneous cervicothoracic epidural hematoma following sit-ups. The hematoma spontaneously resolved following administration of Factor VIIa (168). Due to these reports, some advocate expectant management. Conservative management with serial neurological monitoring and intravenous steroids is not routine $(186,367)$, but may be considered in those patients demonstrating improvement in neurological function (211). Wagner et al (211) reported a spontaneous epidural hematoma in a patient consuming aspirin; remarkably the hematoma resolved spon- 
taneously. Futawatari (368) administered corticosteroids and hyperosmolar therapy in a patient who spontaneously developed a cervical epidural hematoma secondary to idiopathic thrombocytopenic purpura with a platelet count of 10,000 ; the patient made a good recovery with conservative management.

\section{Cervical Spine}

Cervical spine hematomas have occurred spontaneously, with $(116,166,211$, $357,359,360,362,368)$ or without impaired hemostasis $(116,360,369)$. They have occurred after a cervical epidural procedure $(218,356,359,370,371)$, but these are uncommon. In a retrospective series of 347 fluoroscopically guided cervical interlaminar epidural steroid injections, there were no hematomas (372). In 790 consecutive cervical epidural steroid injections, there were no epidural hematomas (373). Another prospective series investigated complications following 204 blind cervical epidural steroid injections. There were no hematomas or episodes of minor bleeding (374). Safe performance of prior cervical epidural procedures in the same patient does not guarantee prevention of a cervical epidural hematoma, following repeat procedures (218). Cervical subdural hematomas have been reported in association with cervical epidural steroid and upper cervical punctures for myelography $(370,375)$.

As far as minor bleeding is concerned, Furman et al (376) reported an overall vascular penetration rate of $19.4 \%$ (376) in 504 cervical transforaminal epidural injections (376) that were performed with a small 25 gauge needle. Aspiration or blood at the hub had low sensitivity in detecting occult vascular puncture. There were no major adverse neurological sequelae. This figure may even under-report the rate of vascular penetration due to the rapidity with which contrast injections wash out.

\section{Thoracic, Lumbar, Sacral Spine}

Neuraxial procedures are safe to perform $(4,5,20)$ and spinal hematomas are rare: the calculated incidence is $1: 150,000$ to $1: 190,000$ for epidurals and $1: 220,000$ for spinals $(5,20,206,207)$. There are several large studies of over 10,000 patients without a single spinal hematoma (66, $208,378-383$ ). Some series of over 100,000 patients reported either one spinal hematoma or none at all (206, 384-386).
During neuraxial needle or catheter placement for orthopedic surgery, several factors influenced the development of minor bleeding (224). Patient-specific factors include female gender, advanced age (>65), prior history of excessive bleeding/ bruising, and hip surgery. Technique-specific variables included type of neuraxial anesthetic (continuous spinal> continuous epidural $>$ single-dose spinal), size of needle gauge (larger than $22>22>$ smaller than 22), multiple passes of the needle (more than $3>2-3>1$ ), and difficulty with needle placement (224). Age and technique (epidural) may be predictive of minor hemorrhagic complications (223).

Minor bleeding following epidural catheterization has been reported in many series $(222,224)$ and confirmed in cadaveric studies (387). Ten post-mortem spines (387) of patients that received epidural catheters were examined. These patients died from causes unrelated to the epidural and had no previous spinal surgery. Six had slight epidural hemorrhages and one had a macroscopically visible hematoma. The latter patient had thrombocytopenia. The hematoma extended over 2-3 segments and was only a few days old (387). Hemorrhages following epidural procedures have been confirmed at laminectomy (388) and during epiduroscopy (389). Usubiaga (388) found significant epidural hemorrhages in $6 \%$ of patients undergoing laminectomy under epidural anesthesia. Olsson and Blomberg (389) reported hemorrhaging following epidural catheter placement under epiduroscopic visualization (389). Minor spinal bleeding may be more frequent than that reported. Rates of vascular puncture as high as $9-11 \%$ have been reported, following epidural catheter placement (390-391).

Aspiration or the spontaneous appearance of blood at the needle hub may underreport vascular puncture (392-394). Furman et al (394) reported an overall vascular penetration rate of $11.2 \%$ (394) in 761 lumbosacral transforaminal epidural injections: $21.3 \%$ at $S 1$ and $8.1 \%$ at the lumbar levels. Renfrew et al (393) reported a $9.2 \%$ incidence of vascular uptake, despite negative aspiration for blood, during caudal epidural procedures. Sullivan et al (392) reports similar rates of vascular uptake in their series of fluoroscopically guided lumbar transforaminals: $10.9 \%$. Caudal epidural rates of vascular uptake are $10.9 \%$. The rates of vascular uptake for sacro-iliac, intra-articular facet, and midline interlaminar epidural steroid injections are $6.1,5.3$, and $1.9 \%$, respectively (392). Patients over the age of $50 \mathrm{dem}$ onstrated twice the rate of vascular uptake as those under 50 (392). Overall, several series report that percutaneous, fluoroscopically-guided, contrast-enhanced, lumbosacral spine injections do not result in clinically significant hematomas, despite significant rates of vascular uptake (392, 394-396).

The relevance of minor hemorrhagic complications in predicting the development of a spinal hematoma remains unresolved $(199,223)$. In one series, however, $62 \%$ of hematomas were associated with a difficult or bloody lumbar puncture: minor bleeding (199). The ability of vascular uptake to predict major bleeding is unknown. Nonetheless, vascular trauma signifies vessel injury and even relatively small volumes of blood can cause significant neural compression (251). If the rate of bleeding outstrips the rate at which the hematoma can evacuate itself, the rate at which clotting can stop ongoing hemorrhage, or the rate at which fibrinolysis occurs, significant neural compression can occur.

In a study of patients undergoing magnetic resonance imaging after an epidural blood patch procedure for postdural puncture headache, Vakharia et al (397) demonstrated that a $20-\mathrm{ml}$ blood patch would produce a hematoma in the posterior epidural space. The mean spread of a $20-\mathrm{ml}$ blood patch was 4.6 intervertebral spaces. None of these patients had radicular symptoms during or after the blood patch procedure. In addition, the report did not note the development of spinal stenosis as a result of the blood patch. Another study evaluating the magnetic resonance imaging patterns of epidural blood patches demonstrated that a focal hematoma mass forms at the injection site. This mass initially compresses the thecal sac and nerve roots, but resolves by 7 hours. Blood patches spread primarily cephalad and only for 3-5 segments (398). Despite the purported safety of epidural blood patches in volumes as high as $30 \mathrm{cc}$, cases of delayed spinal cord/cauda equina have occurred following an epidural blood patch (399). In both cases the hematoma was found to be subdural. However, one could postulate that in patients with reduced epidural space volumes, secondary to neurolysis, prior spinal surgery, degenerative spine conditions, or other spine 
abnormalities, the volumes used in epidural blood patches might not be tolerated (111). In fact, volumes as low as $5 \mathrm{cc}$ of local anesthetic have caused epidural compression at T12-L1, cephalad to a pre-existing extradural compression at L3-4 disc herniation in a patient receiving an epidural steroid injection (400).

Subarachnoid anesthesia is associated with minor degrees of vascular trauma, particularly if paresthesias or technical difficulties are encountered during needle placement (229). Erythrocyte counts were elevated in $38 \%$ of cerebrospinal fluid samples during subarachnoid puncture with a 25 gauge needle (229). Radicular vessels may become damaged, especially if a paresthesia is elicited $(182,229)$. Some authors contend that a 'bloody tap' can be due to arterial puncture (266).

Bleeding occurs not only from catheter insertion but from indwelling catheter movement $(350,387)$. In fact, continuous spinal techniques have been associated with elevated erythrocyte counts in the cerebrospinal fluid (349). Several patients had blood tinged cerebrospinal fluid, but none developed a spinal hematoma (349). Horlocker et al (224) demonstrated that intrathecal catheters had a higher incidence of minor bleeding. This suggests that indwelling neuraxial catheters result in trauma to spinal vasculature and the provision of anticoagulation theoretically increases this risk (203). Spinal cord stimulation has been associated with a delayed hematoma in one report, but this was a surgically implanted subarachnoid plate electrode in the cervical spine (401). If an indwelling intrathecal or epidural catheter increases the risk of minor spinal bleeding, then intrathecal pumps, spinal cord stimulators, and epidural adhesiolysis procedures may have similar problems.

Despite the incidence of vascular trauma with epidural procedures, spinal hematomas are exceedingly rare. One hypothesis that explains why some epidural hematomas spontaneously resolve may account for why most episodes of minor spinal bleeding do not become clinically significant: a hematoma can spread upwards, downwards, or laterally, and thereby, decompress itself $(211,360,367)$. Acute fluid loculations due to an epidural bleed or injectate can lead to neurological compromise, but as the fluid collection resorbs, neurological symptoms can improve (400). In the case of minor spi- nal bleeding, a microscopic bleed may decompress itself and microscopic clot formation may prevent further bleeding.

Subdural hematomas may also develop following neuraxial procedures $(355,399,402-404)$. The subdural space is a potential space and a subdural space occupying lesion can cause significant neurological compromise $(400,405)$. Bleeding into the subdural space, unlike the epidural space, cannot decompress itself away from neurological structures.

Significant spinal hematomas can occur during needle/catheter placement or catheter removal (208). Vandermeulen et al (208) identified 61 published cases of spinal hematoma, from 1906 to 1994, that were associated with neuraxial techniques. Forty six were associated with an epidural technique and 15 with a spinal technique. Thirty (50\%) needle and catheter placements were either technically difficult or demonstrated blood at the needle hub. Forty-two of these 61 patients had evidence of impaired hemostasis. Overall, $87 \%$ of patients that developed spinal hematomas had clotting abnormalities or procedural difficulties. Simplistically, spinal hematomas can occur due to factors that are patient-specific, technique-specific, or unknown.

Wulf (207) identified 51 cases of spinal hematoma due to epidural anesthesia alone. Sixteen of these cases were missed by Vandermeulen (208) or published in the period 1994-1995. The level of epidural anesthesia was known in 47 patients. At the cervical, thoracic, and lumbar levels, there were 2, 9, and 36 hematomas, respectively. Thirteen patients developed cord compression after removal of an epidural catheter. The procedure was difficult, traumatic, or associated with epidural vein trauma in 22 patients. The main risk factors were coagulopathies and anticoagulant therapy, with the exception of aspirin, non-steroidal anti-inflammatory drugs, and subcutaneous, low-dose, unfractionated heparin.

Spinal abnormalities, such as spina bifida oculta, spinal tumors, and ankylosing spondylitis, increase the risk of significant bleeding during neuraxial procedures $(203,207,208)$. Ankylosing spondylitis may increase the risk of bleeding for several reasons: 1. Higher incidence of traumatic attempts due to anatomical abnormalities; 2. Consumption of NSAIDs; 3. Narrower volume of epidural space with smaller foramina (207). Female gen- der and advanced age are risk factors for the development of an epidural hematoma (208). Cutaneous angiomas may be a risk factor the development of epidural hematomas following neuraxial procedures (406). Cutaneous angiomas may indicate the presence of a hazardous lesion in the neuraxis: twenty percent of cutaneous angiomas have associated spinal arteriovenous abnormalities in the same metamere (406). Overall, the applicability of these spinal abnormalities to nonneuraxis procedures is unknown.

\section{Technique Specific Bleeding Risk FACTORS}

We have defined the risk of significant bleeding from an interventional pain procedure based on several factors: 1 . The target's proximity to an important vascular structure; 2 . The target's proximity to a major neurological structure; 3 . The target's location within a confined versus non-confined space; 4 . The use of a sharp rather than blunt needle; 5 . The anticipated or actual need for multiple passages; 6 . The use of fluoroscopy; 7. The use of radiopaque contrast; 8 . The use of a large rather than small diameter needle; 9. The procedure is continuous and indwelling rather than a single shot; 10 . The presence of blood, spontaneously at needle hub or following aspiration.

These factors represent those that influence the absolute risk of bleeding (sharp needle, multiple passes, large needle gauge), the severity of bleeding (proximity to vascular structures), the consequences of bleeding (proximity to neurological structures, target in a confined space), and whether bleeding is recognized (contrast not used, aspiration not performed, fluoroscopy not used, blood at needle hub). Together these factors help guide the clinician in the predicting, detecting, and differentiating bleeding episodes that are less significant, e.g., hematoma after a superficial myoneural injection, versus those that are more significant, e.g. puncturing the aorta with a large bore, sharp needle, during a lumbar sympathetic block.

\section{Is the target structure close to a major vascular or neurological structure?}

If the intended target during a percutaneous procedure for pain therapy and regional anesthesia is or is near a major neurological or vascular structure, the risk and consequences of bleeding may be 
increased. This concept applies to most procedures in interventional pain management. Published reports are limited, but most support this concept.

As described earlier, peribulbar blocks may have fewer complications than retrobulbar blocks, because they are easier to perform and because there are fewer vital structures close the needle (296, 297). Interscalene blocks or infusions may induce a Horner's syndrome (407, 408), but in rare cases this complication may be prolonged, due to traumatic injury or hematoma $(407,408)$. In one case, an expansile hematoma, contained between the anterior scalene and longus colli muscles, compressed and damaged the pre-ganglionic sympathetics. The resulting Horner's syndrome recovered after one year (407, 408). Overall, hematomas following interscalene blocks are rare $(314,407,408)$, but seizures and other signs of local anesthetic toxicity, both indirect markers of vascular injury, may occur in 0.2 and $0.6 \%$ of cases, respectively (314).

Stellate ganglion blocks are high risk procedures. Entry into the vertebral artery can occur in the presence of negative aspiration $(301,305)$. Acute hematomas, causing severe airway obstruction and a locked-in syndrome, have been reported as a consequence of a stellate ganglion block $(301,305)$. This procedure puts vital neural structures at risk as well (409-412).

\section{Is the target in a confined space?}

Bleeding is classified as major if it is intracranial, intraspinal, intraocular, mediastinal, retroperitoneal, or results in death, hospitalization, or transfusion (5). Spinal hematomas are emergent problems because of their location in a confined space. This increases the significance of bleeding, since neural structures can be adversely affected $(5,20,207,208)$. Significant bleeding following lumbar plexus or lumbar sympathetic blocks has primarily resulted in worsening pain, anemia, and hypotension, but neurological compromise is less common $(1,145,233)$.

Intracranial hematomas, like spinal hematomas are considered significant. Intracranial or subdural hemorrhages following lumbar puncture, myelography, spinal anesthesia, or iatrogenic dural puncture are rare but do occur (413418). True causal factors are unknown. Cerebrospinal fluid loss through a dural opening leads to a reduction in intracra- nial pressure that is followed by caudal displacement of the brain. Traction and tearing of bridging meningo-cortical vascular structures occur and lead to a hematoma (413). Recognized contributing factors include age-associated cerebral atrophy, previous head trauma, intra-operative hiccups or coughing, dehydration, post-operative hypotension, larger bore needles, valsalva maneuvers, and multiple punctures $(413,419)$. Forseeably, anticoagulation could worsen a hematoma, but most reports do not mention this influence. However, coagulation disturbances, even the use of anti-inflammatories and mini-dose heparin, are known to influence the development and the outcome of spontaneous intracerebral and subdural hematomas $(419,420)$.

Is the procedure going to be performed with a sharp or blunt needle?

Orbital perforation, during the performance of a retrobulbar block, typically ranges from 1.1 in 1,000 to 1 in 16,000 with sharp needles (231). Blunt needles may reduce this risk relative to sharp needles $(296,421)$. A recent prospective study demonstrated the safety, in terms of reducing the risk of sight-threatening complications, of a blunt approach for ocular blocks (231). A sub-tenon, blunt needle is advocated over a sharp needle during a retrobulbar block to minimize the risk of globe injury $(195,231)$.

Blunt needles may increase the incidence of hematomas during the intentional puncture of a blood vessel (301), but this contention has been refuted by experimental studies (422). Blunt needles, in comparison to sharp needles, are less likely to enter a vital structure and produce hemorrhage (422). Blunt needles may be preferable to sharp ones in performing interventional pain procedures $(422,423)$.

Blunt needles are thought to reduce the risk of vascular injury in peripheral blocks. Furthermore, blunt needles permit tactile sensation and a better 'feel' of fascial tissue planes (424). Chelly indirectly suggests that a blunt, larger bore needle may be safer than a sharp, smaller needle (424).

Several factors are implicated in neural injuries secondary to injection needles: needle trauma, extra- and intra-neural hematomas, microvascular injury and violation of diffusion barriers, and the toxic effects of the injected agent $(292,319)$.
Both immediate and delayed-onset neuropraxic symptoms may be explained by hematomas (292). Subsequent edema, compression, and a degenerative cascade are the main reasons for the development of neural ischemia or neuropathy (319).

Needle design, particularly the length and orientation of the bevel, are relevant (319). There is an ongoing debate about the safety of short versus long beveled needles $(291,292,319,425)$. The length of the bevel approximately correlates with the bluntness of a needle. A long beveled needle is sharper and more likely to impale a nerve. Conversely, a short bevel needle is blunter, less likely to impale a nerve, and permits easier tactile appreciation of tissue planes (319). Selander et al (291) found that the frequency of lesions was greater when a long, as opposed to short beveled needle was used: nerve fascicles have a tendency to roll or slide away from the advancing needle point (291). A short, 45 degree needle was recommended for use in regional anesthesia (291). Rice et al (425) demonstrated that when actual impalement occurred, a short beveled needle produces more lesions that are severe and less rapidly repaired. In summary, a short beveled needle will cause more damage if a nerve is impaled, but it is less likely to impale a nerve, when compared to a long beveled needle (319). Overall, blunt needles may enhance the safety of performing percutaneous interventions and minimize the risk of vascular and neural trauma.

How many needle or catheter passes are going to be performed: Single or Multiple?

Rough and repeated probing for paresthesias, especially with a thick and sharp needle can cause nerve damage (292). Multiple needle passes, during subarachnoid blockade, influenced the red blood cell count in samples of withdrawn cerebrospinal fluid (229). The use of multiple injections, during thoracic paravertebral blockade, is thought to increase the risk of complications $(322,323)$. Bilateral lumbar or thoracic paravertebral blockade is associated with a $9 \%$ incidence of vascular puncture versus $5 \%$ for unilateral blocks (320). An increased number of attempts at epidural catheter placement may increase the risk of a hematoma, whether it is the technique style (midline vs. paramedian) or the experience of the interventionalist (426). 
Minimizing the number of needle passes, during a paracervical block, may reduce the risk of a pelvic hematoma (327). Multiple needle passes, during interscalene blocks, posed a greater risk for developing bruising or inflammation, when compared to a single pass; bruising occurred in $32 \%$ and $10 \%$ of subjects, if more or less than 4 needle passes were used, respectively (290). A repeat lumbar sympathetic has been implicated in worsening a retroperitoneal hematoma that was initiated by the first block (233).

Horlocker et al (288) however attests to the safety of repeat transarterial axillary blocks, even when performed a few days later. Traumatic needle insertion during lumbar puncture has been implicated in subarachnoid hematomas (189). A lumbar puncture should be abandoned if the attempts are numerous or difficult (189). Overall, making multiple passes, during an interventional pain procedure may increase the risk of bleeding.

\section{Are fluoroscopy and contrast-} enhancement going to be used?

The need for fluoroscopy and contrast-enhancement during various interventional and regional anesthetic procedures is actively debated. Fluoroscopy and contrast-enhancement reduce the risk and consequences of procedure related bleeding by several means: 1. Minimizing the procedure difficulty and the need for multiple passes; 2. Enabling the precise placement of needle or catheter away from major vascular or neurological structures; 3. Enabling the early recognition of occult vascular trauma.

By providing real-time imaging, fluoroscopy may potentially reduce the need for multiple needle insertions and thus, the probability of vascular injury. For instance, despite literature demonstrating the success of blind epidurals in difficult patients, such as those with previous back surgery (427), fluoroscopy is thought to enhance the safety of these procedures (428). Other technologies may improve upon this concept in terms of facilitating interventional pain procedures: ultrasound, magnetic resonance imaging, and CT Scanning. However, in comparison to fluoroscopy, these methods are not yet in common use or are not as accessible to interventional pain physicians.

The absence of blood aspiration during the performance of an interventional pain procedure does not exclude the pos- sibility of vascular puncture $(326,376$, $392,394)$. Fluoroscopic guidance, contrast-enhancement and even digital subtraction angiography may be better tools in assessing vascular puncture $(393,430)$. Contrast enhancement has identified instances of vascular puncture, when aspiration could not $(376,392-394)$. Digital subtraction angiography may have a greater sensitivity in detecting vascular uptake during a cervical spine transforaminal injection (429). None of these series resulted in a hematoma. There are no data to suggest a correlation between contrast-enhanced vascular uptake and a clinically significant hematoma. In fact, several series recommending the use of contrast-enhanced fluoroscopy for spinal injection procedures were concerned about the loss of clinical effect and of the consequences of the vascular uptake of injectate $(376,392,394)$, as opposed to the development of a significant hematoma. Nonetheless, contrast enhancement of a vessel, during a fluoroscopically-guided interventional pain procedure, implicitly means that vascular injury and bleeding have occurred. Fluoroscopy and contrastenhancement permit recognition of traumatic vessel injury and consequently, may be helpful in the early detection of bleeding complications.

Is the procedure going to be performed with a large or small diameter needle?

Large needles have been used safely in regional anesthesia. 14-gauge needle and 16-gauge catheter were safely used in patients undergoing continuous femoral blockade (230), without the development of hematomas. Nonetheless, smaller caliber instead of larger caliber needles may be safer for most interventional procedures. One may argue that a smaller needle size increases the risk of needle deviation and hence, increases the likelihood of repeated passes, which in turn increases the probability of vascular trauma. Although this contention may be partially true (430), bevel shape and the needle design will play a more important role in steerability (430-432). Additionally, proper needle orientation may overcome the lesser stiffness of smaller gauge needles (431).

Large bore cannulas and vessel dilators are implicated in large hematomas following internal jugular vein cannulation (301). Intentional puncture of dog kidneys resulted in less bleeding with smaller versus larger gauge needles (422). Large gauge needles of similar size, 16 or 18, may lacerate epidural veins in $5-18 \%$ of patients (350). A 22-gauge Tuohy needle is preferable to a larger 16 gauge needle in thoracic paravertebral blocks (433). Smaller needles are thought to enhance the safety of deep eye blocks (195). In fact, a 25-gauge needle during a lumbar sympathetic block is thought to be of little clinical significance, in terms of bleeding risk. Needle size may affect the frequency and severity of nerve injury (425) and smaller caliber needles are advised (291).

\section{Is the procedure continuous and indwelling or a single shot?}

The risk of bleeding may be greater for an indwelling catheter compared to a single-shot procedure. Indwelling catheters may cause ongoing injury to tissues and vascular structures $(5,387)$. Catheter removal can cause vascular trauma or clot dislodgement $(5,434,435)$. Additionally, as mentioned earlier, indwelling subarachnoid catheters have been associated with hemorrhage, although clinical deficits did not develop (349). Most anesthesiologists in United Kingdom consider single shot epidurals to be safer than indwelling epidurals (259). This issue has implications for long term epidural catheters, spinal cord and peripheral nerve stimulators, and intrathecal pumps.

\section{Is aspiration going to be performed to} exclude vascular penetration?

Aspiration signifies that a blood vessel has been punctured. Reliance on the aspiration of blood may underreport the risk of potential bleeding problems in areas with high vascularity (436). Aspiration, nonetheless, is routinely used to ascertain vascular puncture during regional anesthesia and interventional pain procedures $(294,314,376,392,394)$. If blood is aspirated when performing a regional block, some advocate canceling certain types of surgery $(267,322$, 323). Blood aspiration during a thoracic paravertebral block was a harbinger of a delayed pulmonary hemorrhage $(322,323)$

\section{Technique-Related Bleeding Risk Score and Stratification}

There is no way to discern the relative importance of each technique-specific factor, so each has been assigned one point (Table 7). We have defined the sum 
of all these points as the Technique-Relat- stratified this risk as minimal (1-4), moded Bleeding Risk, $\mathrm{T}_{\mathrm{BR}}$ (Table 8) We have erate (5-6), and severe (7-10). All proce-

Table 7. Technique-related bleeding risk factors and corresponding score

\begin{tabular}{|l|c|}
\hline Risk factors associated with technique & Score \\
\hline Proximity to significant vascular structures & 1 \\
\hline Proximity to significant neurological structures & 1 \\
\hline Target in a confined space & 1 \\
\hline Use of a sharp, rather than blunt needle to reach target & 1 \\
\hline Multiple passages & 1 \\
\hline Contrast not used, if applicable & 1 \\
\hline Fluoroscopy not used, if applicable & 1 \\
\hline Aspiration not performed or presence of blood at needle hub & 1 \\
\hline Needle size: larger than 20 gauge & 1 \\
\hline Continuous, not single shot procedure & 1 \\
\hline
\end{tabular}

Table 8. Technique-related bleeding risk score $\left(T_{B R}\right)$ and risk stratification

\begin{tabular}{|l|c|c|c|}
\hline Overall score & $0-4$ & $5-6$ & $7-10$ \\
\hline Overall risk stratification & Low & Medium & High \\
\hline
\end{tabular}

dures involving needle insertion are theoretically associated with a risk of bleeding, so the minimal $\mathrm{T}_{\mathrm{BR}}$ score should be 1. If the procedure is abandoned, then the $\mathrm{T}_{\mathrm{BR}}$ would be 0 .

Due to the small number of published bleeding complications and the absence of such complications in large series, one cannot authoritatively state that a particular technique poses bleeding risks and consequences that are increased when compared to another technique. However, the $T_{B R}$ alludes to the difficulty and hazards of particular interventional pain techniques. For example, a trigeminal ganglion block, intuitively, poses an increased bleeding risk compared to a superficial trigger point injection, but there is no evidence to support this contention.

Table 9.Patient-related bleeding risk factors and corresponding scores

\begin{tabular}{|c|c|c|}
\hline Hemostasis & Modifying factors & Score \\
\hline Normal & None & 2 \\
\hline Normal & $\begin{array}{l}\text { History of self-limited, transient bleeding } \\
\text { disorder }\end{array}$ & 4 \\
\hline Normal & $\begin{array}{l}\text { Normal coagulation studies despite the intake } \\
\text { of medications that theoretically may affect } \\
\text { hemostasis }\end{array}$ & 6 (nutraceuticals, serotonin reuptake inhibitors) \\
\hline Normal & $\begin{array}{l}\text { Normal coagulation studies after discontinuation } \\
\text { of known anticoagulants (the score may be } \\
\text { modified, depending on when the drug was } \\
\text { stopped relative to the period of drug effect) }\end{array}$ & $\begin{array}{l}\text { 6-10 } \\
6 \text { (e.g., warfarin was stopped } 5 \text { days earlier, aspirin was stopped 7-10 days } \\
\text { earlier, heparin infusion held for }>6 \text { hours) } \\
8 \text { (e.g., aspirin was stopped } 3 \text { days earlier) } \\
10 \text { (e.g. warfarin was stopped } 2 \text { days earlier, heparin infusion was stopped } 4 \\
\text { hours earlier) } \\
\text { 6-10 (e.g., factor or blood product replacement therapy in specific acquired and } \\
\text { congenital bleeding disorders) }\end{array}$ \\
\hline Abnormal & $\begin{array}{l}\text { Active consumption of anticoagulants that } \\
\text { cannot be held (the score may be modified based } \\
\text { on the specific anticoagulant and abnormal } \\
\text { coagulation studies) }\end{array}$ & $\begin{array}{l}10 \text { (low dose aspirin, NSAIDS) } \\
12 \text { (subcutaneous heparin, low dose coumadin (INR < 1.4), medium-high dose } \\
\text { aspirin, ticlopidine, clopidogrel) } \\
14 \text { (low molecular weight heparin, coumadin (INR 1.5-2, Gp IIb/Gp IIIla inhibitors) } \\
16 \text { (intravenous heparin bolus, coumadin (INR 2-3)) } \\
16-18 \text { (thrombin inhibitors) } \\
18 \text { (high dose intravenous heparinization and warfarin, INR >3). } \\
20 \text { (thrombolytics) }\end{array}$ \\
\hline Abnormal & $\begin{array}{l}\text { Known history of medical bleeding disorder (the } \\
\text { score may be modified if there is a history of easy } \\
\text { bruisability, deep versus superficial bleeding } \\
\text { episodes, or spontaneous versus traumatically- } \\
\text { induced bleeding episodes) }\end{array}$ & $\begin{array}{l}10 \text { (thrombocytopenia }>80,000 \text { ) } \\
12 \text { (thrombocytopenia }<80,000 \text {, idiopathic thrombocytopenic purpura, renal } \\
\text { failure-uremia) } \\
12-14 \text { (von Willebrand disease, depending severity) } \\
14 \text { (vitamin K deficiency } \\
14-18 \text { (Hemophilia A and B depending on severity of factor deficiency) } \\
14-18 \text { (liver disease, depending on severity) }\end{array}$ \\
\hline Abnormal & $\begin{array}{l}\text { Known history of significant bleeding with } \\
\text { procedures but cause not identified }\end{array}$ & 18 \\
\hline Abnormal & $\begin{array}{l}\text { Major hemorrhage due to incompetent } \\
\text { coagulation system }\end{array}$ & 20 (disseminated intravascular coagulation) \\
\hline
\end{tabular}




\section{Patient-Related Bleeding Risk Factors}

In a fashion similar to the $\mathrm{T}_{\mathrm{BR}}$, a patient-related bleeding risk score, $\mathrm{P}_{\mathrm{BR}^{\prime}}$ may be generated. This risk of bleeding, simplistically, depends on factors that influence hemostasis: is hemostasis normal or abnormal? (Table 9)

Normal coagulation can be classified as normal, normal with a history of a self-limited, short-lived bleeding disorder, normal coagulation studies (all studies) despite drug intake (herbal medicines), and finally, normal coagulation after discontinuing anticoagulants that normally affect laboratory coagulation studies.

Abnormal coagulation can be broadly classified as patients taking a therapeutic anticoagulant that have not or cannot be discontinued, patients with a history of a medical bleeding disorder, a history of significant bleeding problems following a procedure wherein the cause was not identified, and a major bleeding diathesis.

We have arbitrarily multiplied these patient-specific bleeding risk factors by 2 . Our rationale for weighing patient-specific factors over technique-specific factors was gleaned from the neuraxial anesthesia literature and practice patterns at our institution. Due to the safety record of neuraxial blocks, anticoagulants are thought to be the underlying causal factor in epidural hematomas. This causal link, however, cannot be proven due to the rarity of these events: 1:220,000 in spinal anesthetics and 1:150,000 in epidural anesthetics. The paucity of bleeding complications associated with interventional pain procedures further support this rationale. One caveat is that there may be a selection bias: patients on anticoagulants may be offered non-interventional pain management strategies. Nonetheless, patient specific factors may be more relevant than technique specific factors. The total value is the $\mathrm{P}_{\mathrm{BR}}$ (Table 10).

The risk of $\mathrm{P}_{B R}$ may be defined as mild, moderate, severe, or very severe if the corresponding range of values is 2 to 8,10 to 12,14 to 16 , or 18 to 20 , respectively. A maximal value of 20 represents the significant risk of spontaneous bleeding in patients receiving thrombolytic therapy or suffering from acute disseminated intravascular coagulation. A minimal value of 2 signifies that even in the presence of normal coagulation patients may bleed unpredictably or have a focal lesion that predisposes to bleeding such as an arterio-venous malformation.

General risk factors for major bleeding during anticoagulation, specifically oral anticoagulation, include the intensity of the anticoagulant effect, increased age, female gender, history of gastrointestinal bleeding, length of therapy, and concomitant aspirin usage (138). Aspirin use and the intensity of anticoagulation have been incorporated in our patient-specific score. Increased age, female gender, and length of anticoagulation were not included, but may be considered after the total bleeding risk score is tallied. Elderly age and female gender increase the risk of an epidural hematoma in the presence of certain anticoagulants (5).

Tools to guide clinicians in assessing bleeding risk in patients have been developed. The Outpatient Bleeding Risk Index (437) can distinguish between low, moderate, and high-risk patients and help guide the optimal duration of oral anticoagulant therapy. This index assigns 1 point for age $>65$, history of gastrointestinal bleeding, history of stroke, or the presence of a co-morbid condition (myocardial infarction, anemia, renal impairment, diabetes mellitus). A score of 0 is low risk and a score of 1 or 2 is moderate risk. A score that is 3 is high risk (437). In a prospective study, major bleeding episodes occurred in $3 \%, 12 \%$, and $53 \%$ of patients in the low, moderate, and highrisk categories (437). Physician assessment of bleeding risk was no better than chance in this study (437). This bleeding risk tool may have prevented major bleeding in patients at high risk (437). Another prospective study demonstrated the Outpatient Bleeding Risk Index could distinguish between low and moderate risk patients, in terms of major bleeding (438). The generalizability (438) to other anticoagulants, to surgical procedures, and to interventional pain is unknown, but this index sets a precedent.

Table 10. Patient-related bleeding risk score $\left(P_{B R}\right)$
\begin{tabular}{|l|l|l|l|l|}
\hline Overall score & $2-8$ & $10-12$ & $14-16$ & $18-20$ \\
\hline Overall severity & Mild & Moderate & Severe & Very Severe \\
\hline
\end{tabular}

$\overline{\text { Table 11. Overall significant bleeding risk score in interventional pain practice }}$ $\left(\mathrm{O}_{B R}\right)$ and risk stratification

\begin{tabular}{|l|l|l|l|l|}
\hline Overall score & $2-7$ & $8-14$ & $15-20$ & $21-30$ \\
\hline Overall risk & Low & Medium & High & Very High \\
\hline
\end{tabular}

Overall, our classification attempts to provide a practical and straightforward way of organizing the most common, patient-specific factors that influence hemostasis.

\section{Risk Stratification}

One can then stratify overall risk of significant bleeding $\left(\mathrm{O}_{\mathrm{BR}}\right)$, i.e., bleeding that may result in significant morbidity, by adding patient-related bleeding risk score, $\left(\mathrm{P}_{\mathrm{BR}}\right)$ and technique-related bleeding risk score $\left(\mathrm{T}_{\mathrm{BR}}\right)$. ble 11)

In summary, $\mathrm{O}_{\mathrm{BR}}=\mathrm{T}_{\mathrm{BR}}+\mathrm{P}_{\mathrm{BR}}(\mathrm{Ta}-$

The range of values for $\mathrm{O}_{B R}$ is 230 (Table 8). Low risk of bleeding would be $\leq 7$, medium risk would be $8-14$, high would be 15-20, and very high risk would be $\geq 21$. Recall that we have weighted patient over technique specific factors, by a factor of two. of the most invasive techniques, the best

possible $T_{B R}$ may fall between 3 and 4 . If these techniques are not optimized, then the score could deteriorate to 10 . At our institution, this type of procedure, even if optimally performed would be postponed if the patient consumed aspirin or an NSAID (a score of 10). Many clinicians may disagree with this decision and proceed with this procedure. Due to this controversy, we have classified this $\mathrm{O}_{\mathrm{BR}}$ score of 13 to $14(10+3$ or $10+4)$ as medium risk. If the procedure is performed

haphazardly, then the $\mathrm{O}_{\mathrm{BR}}$ could increase to 20: high risk.

If the patient consumed ticlopidine or clopidogrel (a score of 12), the decision to cancel the case would be less contro-

versial. In this case the $\mathrm{O}_{B R}$ score would be 15 to $16(12+3$ or $12+4)$ and we would classify this as high risk. If the patient was
If one optimizes the performance 
anticoagulated with coumadin and the INR was in the 2-2.5 range (a score of 16),

the $\mathrm{O}_{\mathrm{BR}}$ score would be 19 to $20(16+3$ or $16+4)$ : high to very high risk. The procedure should not be performed.

\section{Examples}

1. What would the $\mathrm{O}_{B R}$ and risk stratification be in a patient that undergoes a blind trigeminal ganglion block with a sharp 22-gauge needle? Unwittingly, this patient has been receiving low molecular weight heparin twice-daily and the last dose was given two hours earlier.

The $\mathrm{T}_{\mathrm{BR}}$ would be 7 and this risk stratification would be high:

+1 (proximity to vascular structures-intracranial vessels)

+1 (proximity to neurological structures-brain)

+1 (confined compartment-middle cranial fossa)

+1 (use of a sharp needle)

+3 (blind technique implies no use of contrast or fluoroscopy and the need for multiple passes)

+0 (standard practice for this block is that aspiration is required) $<20$

+0 (use of a needle with a gauge

+0 (single shot procedure)

The $\mathrm{P}_{\mathrm{BR}}$ score would be 14 and this risk stratification would be severe.

Hence the $\mathrm{O}_{\text {BR }}$ would be $21(14+7)$ : very high risk for bleeding related complications.

The peak effect of subcutaneous low molecular weight heparin occurs at about two hours. There have been cases of epidural hematoma when the catheter was pulled at this time after dosing of low molecular weight heparin. Additionally low molecular weight heparin that is dosed at BID has been associated with a high risk of hematoma. Since monitoring PT doesn't correlate with bleeding, one must closely monitor the patient for a facial hematoma, neurological changes, specifically those in the middle cranial fossa (cranial nerves 4, 6, and 3), seizures, and an elevated ICP. A small amount of blood can rapidly cause clinical demise. Emergency imaging, such as a CAT scan or MRI should be performed to exclude a hematoma and neurosurgical consultation should be sought. Like wise standard Advanced Cardial Life Support. protocols should be initiated in the event of rapid neurological decline. Neuroprotective and other pharmacological agents may be necessary to treat seizures or the delayed sequelae of an intracranial hematoma. We would advise delaying the case for 24 hours and perhaps, 48 hours in order to minimize residual effects of the low molecular weight heparin. Protamine would not be effective in reversing the effects of low molecular weight heparin. Even if contrast and fluoroscopy were used the $\mathrm{O}_{B R}$ would be 19: high. Hence, even if technique-related strategies were used to reduce the $\mathrm{O}_{\mathrm{BR}}$, the technique should be postponed.

2. What would the $\mathrm{O}_{B R}$ and risk stratification be if a patient stopped aspirin 7 days earlier and will be undergoing a trial of thoracic spinal cord stimulation?

The technique specific risk score would be 8 and this risk stratification would be high:

+1 (proximity to vascular structures- epidural veins)

+1 (proximity to neurological structures- spinal cord)

+1 (confined compartment- spinal canal)

+1 (Tuohy needle has a rounded edge, but this edge is sharp and has the ability to pierce skin)

+2 (Fluoroscopy is used, but many practitioners do not use contrast; multiple passes are needed to steer the electrode into position, by definition)

+0 (standard practice for this procedure is that aspiration is required)

+1 (use of a needle with a gauge $>20$ the standard needle for spinal cord stimulators is 14 or 15 gauge). trode)

+1 (continuous and indwelling elec-

The patient specific risk score would be 6 and this risk stratification would be mild. Hence, the $\mathrm{O}_{\mathrm{BR}}$ score would be 14 $(8+6)$ : medium risk.

The irreversible effect of platelet inhibition associated with aspirin will normalize in 7-10 days. The patient should be asked to stop the aspirin and return in 10 days, 3-5 days may be appropriate for NSAIDs. As for Cox-II inhibitors, platelet assays suggests they have no anti-platelet effects. Some practitioners may obtain a bleeding time or platelet function assay, but these studies may not guarantee the prevention of bleeding. Several studies demonstrate that bleeding time measurements following anti-platelet therapy are not a reliable indicator of plate- let function, degree of antiplatelet effect, nor surgical blood loss $(25,29,90,439)$. Platelet function analysis as measured by the platelet response to adenosine diphosphate or epinephrine can take up to 3 or 7 days to normalize, with non-steroidals or aspirin respectively, even if the bleeding time is normal $(5,81)$. Platelet function analyses may be useful under such circumstances. Finally, the technique-related bleeding risk score may be reduced by using contrast.

If the procedure is planned and aspirin or non-steroidal anti-inflammatory agents have been administered, postponing the procedure should be considered. Although the ASRA guidelines do not consider aspirin and NSAIDs to be a contraindication to neuraxial anesthesia, spinal cord stimulation is different from a standard epidural catheter in several respects: 1 . Spinal cord stimulators are implanted for 5-7 days in the case of a trial and indefinitely for a permanent implant; 2. Spinal cord stimulation precludes the use of magnetic resonance imaging, which is considered the gold standard for diagnosing a spinal epidural hematoma; 3. A significant proportion of patients that are selected for spinal cord stimulation have had prior spinal surgery, which causes epidural scarring and fibrosis (428); 4. Spinal cord stimulator leads are stiffer than most epidural catheters; and 5. Needles used for spinal cord stimulation lead insertion are larger in caliber compared to those used for most epidural catheters used in regional anesthesia. Desmopressin and platelet concentrates have been used to reverse the effects of antiplatelet therapy that cannot be held, but this seems excessive for an elective procedure like spinal cord stimulation.

3. What would the $\mathrm{O}_{B R}$ and risk stratification be for the performance of a lumbar sympathetic block, using contrast-enhanced fluoroscopy and a 20gauge blunt needle in a patient with a history of peripheral vascular disease that suffers an acute thrombotic event with impending ischemia? The patient is receiving thrombolytic therapy and systemic heparinization.

The technique specific risk score would be 3 and this risk stratification would be low:

+1 (proximity to vascular structures-aorta or inferior vena cava)

+1 (proximity to neurological structures-lumbar plexus) 
+0 (compartment is not confined)

+0 (use of a blunt needle)

+0 (contrast and fluoroscopy are used and only a single pass is necessary for a block)

+0 (standard practice for this block is that aspiration is required)

+1 (the needle size is 20 gauge).

+0 (the procedure is a single shot)

The patient specific risk score would be 20 and this risk stratification would be very severe. Hence the $\mathrm{O}_{\text {BR }}$ score would be 25: very high risk. One should cancel the case and wait at least 10-14 days. In reality, no one knows how long interventional pain procedures should be held following thrombolytics. If the block must be performed, the practitioner must closely coordinate the block with the primary team. Thrombolytics should be held, fibrinogen levels should be monitored, and fresh frozen plasma may have to be administered. We cannot advise when thrombolytics can be re-started. Heparin may be restarted 12 hours after the block and if possible, no bolus should be administered. Close serial neurological monitoring should be performed at an interval $\leq 2^{\circ}$. Since the block is going to be used for improving limb perfusion, the use of long acting and potent local anesthetics may be unavoidable. For this reason, one may wish to consider the use of a radiofrequency neurolysis instead.

The $\mathrm{O}_{B R}$ score in interventional pain practice incorporates the risks of bleeding secondary to both patient and technique-specific factors. This score can help the clinician in terms of deciding whether to perform or to abandon an interventional pain procedure. No firm rules can be made as to whether one should abandon a very high-risk procedure or to continue with a low-risk procedure. Herein lay the importance of individual judgment and assessment of the risk-benefit ratio. Nonetheless, strategies to reduce the

$T_{B R}$ score should be universally adopted. Holding anticoagulants, substituting alternative anticoagulants, or reversing anticoagulation, in order to reduce the $\mathrm{P}_{\mathrm{BR}}$ score, should be carried out in consultation with the physician managing the patient's anticoagulation. If a strategy to re-

duce the $\mathrm{P}_{B R}$ score is employed, the patient must be fully informed of the potential risks. Interventional pain practitioners must understand that predicting a significant bleeding event in the end is impossible and a complication may occur by chance. Herein are the limitations of both the $\mathrm{O}_{B R}$ score and individual physician judgment.

Management strategies for bleeding complications have been alluded to in this monograph, but a discussion about managing specific bleeding problems following interventional pain procedures are beyond the scope of this paper. The mention of a specific case, however, would be useful.

Conolly et al (440) published a case report, in 1995, on the management of a 40-year old patient with Hemophilia A that was pending an epidural steroid injection. Factor VIII levels ranged from 4$8 \%$. The patient was diagnosed with hemophilia at the age of 23 after developing an intra-abdominal hemorrhage that required surgery. Hemarthroses in the left hip led to a progressive arthropathy that ultimately required hip replacement surgery. Factor VIII concentrate was used perioperatively with success.

The consultant hematologist advised measuring factor VIII levels prior to the epidural steroid injection. The hematologist also suggested tests to assess if factor VIII inhibitors were present. Thereafter, factor VIII concentrate should be administered in order to raise Factor VIII levels to as close to $100 \%$, as possible. Thereafter, a repeat factor VIII level should be performed. After the epidural injection, factor VIII concentrates should be continued as an in-patient for 24 hours and a repeat MRI should be performed prior to discharge. The procedure was performed with a 19-gauge Tuohy needle and with the use of contrast-enhanced fluoroscopy. There was no mention of technical difficulties or the aspiration of blood. The procedure was repeated a few weeks later using the same protocol. The cost of one epidural steroid injection with hematological management in 1995 dollars was over $\$ 34,000$ (440).

The technique specific risk score would be 5 and this risk stratification would be medium:

+1 (proximity to vascular structures-epidural veins)

+1 (proximity to neurological structures-cauda equina)

+1 (compartment is confined)

+1 (use of a Tuohy needle)

+0 (contrast and fluoroscopy are used and only a single pass is necessary for a block)

+0 (standard practice for this block is that aspiration is required)

+1 (the needle size is 19 gauge).

+0 (the procedure is a single shot)

The patient specific risk score would be 16: factor VIII levels were mildly ( $8 \%)$ to moderately $(4 \%)$ reduced. This risk stratification would be severe. If the procedure were performed in this setting, the $\mathrm{O}_{\mathrm{BR}}$ would be $21(16+5)$, i.e., very high. However, with factor VIII replacement to nearly $100 \%$ and the absence of inhibitors, one could argue that the patient's hemostatic capabilities were restored to 'normal'. We would assign a $\mathrm{P}_{\mathrm{BR}}$ of 6 . Thus, the $\mathrm{O}_{\mathrm{BR}}$ would be $11(6+5)$, i.e., medium risk.

\section{ConcLusion}

Significant bleeding following an interventional pain procedure is an extremely rare event. Tools to help predict these events would be helpful to practitioners. Unfortunately, available information is scarce and the number of patients needed in a clinical trial, to answer questions about which factors pose a risk for bleeding, would be staggering. Nonetheless, this problem can be approached by making some assumptions, i.e., the risk of significant bleeding is dependent on the underlying hemostatic problems and the particular technique used. Independent risk factors for perioperative bleeding, such as advanced age or sex, have not been included in stratifying the risk of bleeding with interventional pain procedures. However, they should not be ignored.

An overall bleeding risk score can be generated based on a stratification of technique-specific and patient-specific factors. Strategies can be implemented to reduce the technique- and patient-specific risk factors, in order to reduce the overall bleeding risk score. Such strategies may help in decisions to avoid, abort or proceed with a procedure.

Although not validated, the overall bleeding risk score is based on an exhaustive review of the literature: this literature pertains to hemostasis and interventional pain procedure-related bleeding. Use of this overall bleeding risk assessment tool may help practitioners in reducing the risk of significant bleeding with interventional pain and regional anesthetic procedures, in improving patient safety, and in research-oriented data collection. 


\section{ACKNOWLEDGEMENT}

We kindly thank Margaret Vugrin, MLA, a reference librarian at the Preston Smith Library at Texas Tech University Health Sciences Center, for her help in the literature search. We also thank Dr. Everardo Cobos, Associate Professor and Chief of the Hematology and Oncology Division, Dept. of Internal Medicine, Texas Tech University Health Sciences Center for his input.

\section{REFERENCES}

\section{Author Affiliation: \\ P. Prthvi Raj, MD}

Chairman, World Institute of Pain

Section of Pain Practice

4748 Matterhorn Way

Antioch, CA 94521

E-Mail:prithviraj7@comcast.net

Rinoo V. Shah, MD

Assistant Professor

Dept. of Anesthesiology and Pain

Services

International Pain Institute

Texas Tech University Health

Sciences Center

4430 South Loop 289

Lubbock, TX 79414

E-mail: rinoo_shah@yahoo.com

\section{Alan D. Kaye, MD, PhD}

Chairman and Professor

Dept. of Anesthesiology and Pain

Services

Professor of Pharmacology

$36014^{\text {th }}$ Street

Lubbock, TX 79430

E-mail: Alan.Kaye@ttuhsc.edu

\section{Stephen Denaro, BS}

Dept. of Anesthesiology and Pain

Services

$36014^{\text {th }}$ Street

Lubbock, TX 79430

E-mail: Stephen.Denaro @ttuhsc.edu

Jason M. Hoover, BS

Dept. of Anesthesiology and Pain

Services

$36014^{\text {th }}$ Street

Lubbock, TX 79430

E-mail: Jason.Hoover@ttuhsc.edu
1. Weller RS, Gerancher JC, Crews JC et al. Extensive retroperitoneal hematoma without neurological deficit in two patients who underwent lumbar plexus block and were later anticoagulated. Anesthesiology 2003; 98:581-585.

2. Dunn A, Turpie A. Perioperative management of patients receiving oral anticoagulants: a systematic review. Archives of Internal Medicine 2003; 163; 901-908

3. Llau JV, de Andres J, Gomar C et al. [Drugs that alter hemostasis and regional anesthetic techniques: safety guidelines. Consensus conference]. Rev Esp Anestesiol Reanim $2001 ; 48: 270-278$.

4. Gogarten W, Van Aken H, Wulf $\mathrm{H}$ et al. Para-spinal regional anesthesia and prevention of thromboembolism/ anticoagulation : Recommendations of the German Society of Anesthesiology and Intensive Care Medicine. Anasthesiol Intensivmed Notfallmed Schmerzther 1997 ; $38: 623-628$.

5. Horlocker TT, Wedel DJ, Benzon $\mathrm{H}$ et al. Regional anesthesia in the anticoagulated patient: defining the risks (the second ASRA Consensus Conference on Neuraxial Anesthesia and Anticoagulation). Reg Anesth Pain Med 2003 ;28:172-197.

6. Kroll DA, Caplan RA, Posner K et al. Nerve injury associated with anesthesia. Anesthesiology $1990 ; 73: 202-207$.

7. Cheney FW, Domino KB, Caplan RA et al. Nerve injury associated with anesthesia: a closed claims analysis.Anesthesiology 1999;90:1062-1069.

8. Manchikanti L, Staats P, Singh V et al. Evidence-Based Practice Guidelines for Interventional Techniques in the Management of Chronic Spinal Pain. Pain Physician 2003, $6: 3-81$

9. Ansell J. The Perioperative Management of Warfarin Therapy [Editorial] Arch Intern Med 2003;163:881-883.

10. Guyton AC, Hall JE. Chapter 36: Hemostasis and Blood Coagulation. In: Guyton AC, Hall JE (Ed). Textbook of Medical Physiology $10^{\text {th }}$ Ed. WB Saunders Company, Philadelphia, 2001, pp 419-429.

11. Petrovitch CT, Drummond JC. Hemotherapy and Hemostasis. Barash PG, Cullen BF, Stoelting RK (eds). Clinical Anesthesia. $4^{\text {th }}$ Ed. Lippincott Williams \& Wilkins, Philadelphia, 2001; pp 201-242.

12. Meisenberg G, Simmons WH. Principles of Medical Biochemistry. Mosby Inc, St Louis, 1998; pp 511-545.

13. Cobos E, Cruz JC, Day M. Etiology and management of coagulation abnormalities in the pain patients. Curr Rev Pain 2000; 4: 431-439.

14. George JN, Woolf SH, Raskob GE et al. Idiopathic thrombocytopenic purpura: a practice guideline developed by explicit methods for the American Society of Hematology. Blood 1996 ;88:3-40.

15. Movig KL, Janssen MW, de Waal Malefijt J et al. Relationship of serotonergic antidepressants and need for blood transfusion in orthopedic surgical patients. Arch Intern Med $2003 ; 163: 2354-2358$.

16. Peterson P, Hayes TE, Arkin DE et al. The preoperative bleeding time test lacks clinical benefit: college of American Pathologists' and American Society of Clinical Pathologists position article. Arch Surg 1998, 133:134-139.

17. Whitten CW, Greilich PE Thromboelastography: past, present, and future. Anesthesiology 2000;92:1223-1225

18. Mielke $\mathrm{CH}$. Measurement of the bleeding time. Thromb Haemost 1984; 52:210-211.

19. Mielke CH Jr, Kaneshiro MM, Maher IA et al. The standardized normal lvy bleeding time and its prolongation by aspirin. Blood 1969;34:204-215

20. Tyagi A, Bhattacharya A. Central neuraxial blocks and anticoagulation: $A$ review of current trends. Eur J Anaesthesiol 2002 ;19:317-329.

21. Sauer W, Schwagmeier R, Nolte H. Long term medication with acetylsalicylic acid-a problem in regional anesthesia? Anaesthesist 1992;41:489-493.

22. Macdonald R. Bleeding time. Br / Anaesth 1992;69:329; author reply 330-331.

23. Babson SR, Babson AL.Development and evaluation of a disposable device for performing simultaneous duplicate bleed ing time determinations. Am J Clin Pathol 1978 ;70:406-408.

24. Lind SE. The bleeding time does not predict surgical bleeding. Blood 1991 15;77: 2547-2552

25. Rodgers RPC, Levin J. A critical reappraisal of the bleeding time. Semin Thromb Hemost 1990; 16:1-20.

26. Connelly NR, Hall S. Intraoperative hypotension due to unrecognized bleeding from a continuous spinal catheter site. $J$ Clin Anesth 1993 ;5:497-499.

27. Amrein PC, Ellman L, Harris WH. Aspirininduced prolongation of bleeding time and perioperative blood loss. JAMA 1981 8;245:1825-1828

28. O'Laughlin JC, Hoftiezer JW, Mahoney JP et al. Does aspirin prolong bleeding from gastric biopsies in man? Gastrointest Endosc 1981;27:1-5

29. Hindman BJ, Koka BV. Usefulness of the post-aspirin bleeding time. Anesthesiology 1986; 64:368-370.

30. Favaloro EJ.Utility of the PFA-100 for as sessing bleeding disorders and monitoring therapy: a review of analytical variables, benefits and limitations. Haemophilia. $2001 ; 7: 170-179$

31. Fressinaud E, Veyradier A, Truchaud F, Martin I, Boyer-Neumann C, Trossaert M, Meyer D. Screening for von Willebrand disease with a new analyzer using high shear stress: a study of 60 cases. Blood 1998 15;91:1325-1331.

32. Fressinaud E, Veyradier A, Sigaud M et al. Therapeutic monitoring of von Willebrand disease: interest and limits of a platelet function analyser at high shear rates. $\mathrm{Br}$ 
J Haematol 1999;106:777-783.

33. Kundu SK, Heilmann EJ, Sio R et al. Description of an in vitro platelet function analyzer--PFA-100. Semin Thromb Hemost 1995;21:106-112.

34. Kottke-Marchant K, Powers JB, Brooks L et al. The effect of antiplatelet drugs, heparin, and preanalytical variables on platelet function detected by the platelet function analyzer (PFA-100). Clin Appl Thromb Hemost 1999;5:122-130

35. Fattorutto $M$, Pradier $O$, Schmartz $D$ et al. Does the platelet function analyser (PFA100) predict blood loss after cardiopulmonary bypass? B r J Anaesth 2003 ;90:692693.

36. Cammerer U, Dietrich W, Rampf T et al. The predictive value of modified computerized thromboelastography and platelet function analysis for postoperative blood loss in routine cardiac surgery. Anesth Analg 2003;96:51-57.

37. Forestier F, Coiffic A, Mouton C et al. Platelet function point-of-care tests in post-bypass cardiac surgery: are they relevant? BrJ Anaesth 2002;89:715-721.

38. Cuvillon E, Bonnetty M, Favereau JP et al. Epidural analgesia in a pregnant woman with essential thrombocythaemia. Ann Fr Anesth Reanim 2003;22:453-456.

39. Bigeleisen PE, Kang $Y$ Thrombelastography as an aid to regional anesthesia: preliminary communication. Reg Anesth 1991 ;16:59-61.

40. Kang YG, Martin DJ, Marquez J et al. Intraoperative changes in blood coagulation and thrombelastographic monitoring in liver transplantation. Anesth Analg 1985;64:888-896.

41. Sharma SK, Philip J, Whitten CW et al. Assessment of changes in coagulation in parturients with preeclampsia using thrombo elastography.Anesthesiology 1999;90: 385-390.

42. Kaufmann CR, Dwyer KM, Crews JD et al. Usefulness of thrombelastography in assessment of trauma patient coagulation. J Trauma 1997 ;42716-720.

43. Santoro SA, Eby CS. Laboratory Evaluation of Hemostatic Disorders: Hoffman, Hematology:Basic Principles and Practice, $3^{\text {rd }}$ Ed. Churchill Livingstone, 2000, pp 18411849.

44. Suchman AL, Mushlin Al. How well does the activated partial thromboplastin time predict postoperative hemorrhage? JAMA 1986 8;256:750-753.

45. Hirsh J, Dalen JE, Anderson DR et al. Oral anticoagulants: mechanism of action, clinical effectiveness, and optimal therapeutic range. Chest 1998;114:445S-469S.

46. Hirsh J. Oral anticoagulant drugs. $N$ Engl J Med 1991; 324:1865-1875.

47. Ellison N. Diagnosis and management of bleeding disorders. Anesthesiology. 1977;47:171-180.

48. Mannucci PM. Treatment of von Willebrand disease. Haemophilia. 1998;4:661-

\section{4.}

49. Mannucci PM, Federici AB.Best Management of inherited von Willebrand disease. Pract Res Clin Haematol. 2001;14:455462.

50. Cameron CB, Kobrinsky N. Perioperative management of patients with von Willebrand's disease. Can J Anaesth 1990; 37: 341-347.

51. Fulcher CA, Zimmerman TS. Characterization of the human factor VIII procoagulant protein with a heterologous precipitating antibody. Proc Nat'l Acid Sci USA 1982; 79:1648-1652.

52. Stedeford JC, Pittman JA. Von Willebrand's disease and neuroaxial anaesthesia. Anaesthesia 2000 ;55:1228-1229.

53. Cotran RS, Kumar V, Collins T. Red Cells Bleeding Disorders: Robbins, Pathology of Disease. $6^{\text {th }}$ Ed. Philadelphia, 1999;pp 601-643.

54. Weigert Al, Schafer Al. Uremic bleeding: pathogenesis and therapy. Am J Med Sci 1998, 316:94-104.

55. Dhar P, Abramovitz S, DiMichele D et al. Management of pregnancy in a patient with severe haemophilia $\mathrm{A}$. Br J Anaesth 2003;91:432-435.

56. Meena AK, Jayalakshmi S, Prasad VS et al. Spinal epidural haematoma in a patient with haemophilia-B. Spinal Cord 1998;36: 658-66o.

57. Mannucci PM, Tuddenham EG.The hemophilias--from royal genes to gene therapy. N Engl J Med 2001 ;344:1773-1779.

58. Aldouri M Pathophysiol The use of recombinant factor VIla in controlling surgical bleeding in non-haemophiliac patients. Haemost Thromb 2002;32:41-46.

59. Christie-Taylor, GA and McAuliffe GL. Epidural placement in a patient with undiagnosed acquired haemophilia from factor VIII inhibitor. Anaesthesia 1999;54: 367371.

6o. Morrison AE and Ludlam CA. Acquired haemophilia and its management. British Journal of Haematology. 1995; 54: 231236.

61. Zakarija A, Green D. Acquired hemophilia: diagnosis and management. Curr Hematol Rep 2002;1:27-33.

62. Green D. The management of factor VIII inhibitors in non-hemophilic patients. Progress in Clinical Biological Research 1984; 54: 337-352.

63. Ingerslev J, Freidman D, Gastineau D et al. Major surgery in haemophilic patients with inhibitors using recombinant factor VIla. Haemostasis 1996;26:118-123.

64. Hauser I, Schneider B, Lechner K. Postpartum factor VIII inhibitors. A review of the literature with special reference to the value of steroid and immunosuppressive treatment. Thrombosis and Haemostasis 1995; 54: 1-4.

65. Kane AB, Kumar V. Environmental and Nutritional Pathology: In Cotran RS, Kumar V, Collins T (eds). Robbins, Pathology of Dis- ease. $6^{\text {th }}$ Ed. Philadelphia,1999, pp 403457.

66. Crawford JM. The Gastrointestinal Tract. In Cotran RS, Kumar V, Collins T (eds). Robbins, Pathology of Disease. $6^{\text {th }}$ Ed. Philadelphia, 1999; pp 775-843.

67. Gustafsson H, Rutberg H, Bengtsson M Spinal haematoma following epidural analgesia. Report of a patient with ankylosing spondylitis and a bleeding diathesis. Anaesthesia 1988; 43:220-222.

68. Sallah S, Bobzien W.Bleeding problems in patients with liver disease. Ways to manage the many hepatic effects on coagulation. Postgrad Med 1999 ;106:187-190 \& 193-195.

69. DeLoughery TG: Management of bleeding with uremia and liver disease. Curr Opin Hematol 1999, 6:329-333.

70. Kelly DA, Tuddenham EG. Haemostatic problems in liver disease. Gut 1986;27: 339-349.

71. Messmore HL. Wehrmacher WH. Disseminated Intravascular Coagulation: A primer for primary care physicians. Postgrad Med 2002; 111.

72. Noris M, Remuzzi G. Uremic bleeding: closing the circle after 30 years of controversies? Blood 1999 ;94:2569-2574.

73. Friedmann AM, Sengul H, Lehmann $\mathrm{H}$ et al. Do basic laboratory tests or clinical observations predict bleeding in thrombocytopenic oncology patients? A reevaluation of prophylactic platelet transfusions. Transfus Med Rev 2002;16:34-45.

74. Simon TL, Akl BF, Murphy W. Controlled trial of routine administration of platelet concentrates in cardiopulmonary bypass surgery. Ann Thorac Surg 1984;37:359364 .

75. Ansell JE. In Hemorrhagic and Thrombotic Disorders: Noble, Textbook of Primary Care Medicine, $3^{\text {rd }}$ ed. Mosby, St Louis, Mo:2001;pp 1044-1052

76. Warkentin TE, Kelton JG. Thrombocytopenia Due to Platelet Destruction and Hypersplenism: In Hoffman, Hematology:Basic Principles and Practice, $3^{\text {rd }}$ ed. Churchill Livingstone, 2000: pp 2139-2154

77. Cines DB, Blanchette VS. Immune thrombocytopenic purpura. N Engl J Med 2002 ;346:995-1008

78. Cohen YC, Djulbegovic B, Shamai-Lubovitz $O$ et al. The bleeding risk and natural history of idiopathic thrombocytopenic purpura in patients with persistent low platelet counts. Arch Intern Med 2000;160: 1630-1638.

79. Okutomi T, Taguchi M, Amano K et al. Paravertebral block for labor analgesia in a parturient with idiopathic thrombocytopenia. Masui 2002 ;51:1123-1126.

80. Gerlach R. Raabe A. Zimmermann M. Siegemund A. Seifert V. Factor XIII deficiency and postoperative hemorrhage after neurosurgical procedures. Surgical Neurology 2000 54:260-264; discussion 264-265. 
81. Cronberg S, Wallmark E, Soderberg I. Effect on platelet aggregation of oral administration of 10 non-steroidal analgesics on humans. Scand J Haematol 1984; 33:155159.

82. Greer IA. Effects of ketorolac tromethamine on hemostasis. Pharmacotherapy 1990;10:71S-76S.

83. Leese PT, Hubbard RC, Karim A et al. Effects of celecoxib, a novel cycloxygenase2 inhibitor, on platelet function in healthy adults: a randomized controlled trial. J Clin Pharmacol 2000; 40:124-132.

84. Peters RJ, Mehta SR, Fox KA et al. Clopidogrel in Unstable angina to prevent Recurrent Events (CURE) Trial Investigators. Effects of aspirin dose when used alone or in combination with clopidogrel in patients with acute coronary syndromes: observations from the Clopidogrel in Unstable angina to prevent Recurrent Events (CURE) study. Circulation 2003;108:1682-1687.

85. Reymond MA, Marbet G, Radu EW et al.Aspirin as a risk factor for hemorrhage in patients with head injuries. Neurosurg Rev 1992;15:21-25.

86. Fauno P, Petersen K, Husted S. Increased blood loss after preoperative NSAID. Retrospective study of 186 hip arthroplasties. Acta Orthop Scand 1993;64:522-524.

87. Thurston AV, Briant SL. Aspirin and postprostatectomy haemorrhage. $\mathrm{Br} / \mathrm{Urol}$ 1993;71:574-576.

88. Gunter JB, Varughese AM, Harrington JF et al. Recovery and complications after tonsillectomy in children: a comparison of ketorolac and morphine. Anesth Analg 1995 ;81:1136-1141.

89. Engel C, Lund B, Kristensen SS et al. Indomethacin as an analgesic after hysterectomy. Acta Anaesthesiol Scand 1989;33: 498-501

90. Ferraris VA, Swanson E. Aspirin usage and perioperative blood loss in patients undergoing unexpected operations. Surg Gynecol Obstet 1983;156:439-442.

91. CAPRIE Steering Committee. A randomised, blinded, trial of clopidogrel versus aspirin in patients at risk of ischaemic events (CAPRIE). Lancet 1996 ;348:13291339.

92. Taniuchi M, Kurz HI, Lasal JM. Randomized comparison of ticlopidine and clopidogrel after intracoronary stent implantation in a broad patient population. Circulation 2001; 104:539-543.

93. Schror K. Antiplatelet drugs. A comparative review. Drugs 1995;50:7-28.

94. Yang LH, Fareed J. Vasomodulatory action of clopidogrel and ticlopidine. Thromb Res $1997 ; 86: 479-491$.

95. Pereillo JM, Maftouh M, Andrieu A et al. Structure and Stereochemistry of the active metabolite of clopidogrel. Drug Metab Dispos 2002;30:1288-1295.

96. Clarke TA, Waskell LA. The metabolism of clopidogrel is catalysed by human cytochrome P450 3A and is inhibited by atorvastatin. Drug Metab Dispos 2003;31:53-
59.

97. Bhatt DL, Hirsch AT, Ringleb PA et al. Reduction in the need for hospitalization for recurrent ischemic events and bleeding with clopidogrel instead of aspirin. CAPRIE investigators. American Heart Journal 2000; 140:67-73.

98. Gorelick PB, Born GV, D'Agostino RB, et al. Therapeutic benefit. Aspirin revisited in light of the introduction of clopidogrel. Stroke. 1999;30(8):1716-1721.

99. Tendera M, Wojakowski W. Role of antiplatelet drugs in the prevention of cardiovascular events. Thromb Res 2003 15;110: 355-359.

100. Wilhite DB, Comerota AJ, Schmieder FA et al. Managing PAD with multiple platelet inhibitors: the effect of combination therapy on bleeding time. J Vasc Surg 2003 ;38: 710-713.

101. Yusuf S, Zhao F, Mehta SR et al. Clopidogrel in Unstable Angina to Prevent Recurrent Events Trial Investigators. Effects of clopidogrel in addition to aspirin in patients with acute coronary syndromes without ST-segment elevation. N Engl I Med $2001 ; 345: 494-502$.

102. O'Neill WW, Serruys P, Knudtson M et al. Long-term treatment with a platelet glycoprotein-receptor antagonist after percutaneous coronary revascularization. EXCITE Trial Investigators. Evaluation of Oral Xemilofiban in Controlling Thrombotic Events. N Engl J Med. 2000;342:13161324 .

103. EPIC INVESTIGATORS. Use of a monoclonal antibody directed against the platelet glycoprotein IIb/IIla receptor in high-risk coronary angioplasty. The EPIC Investigation. N Engl J Med. 1994 ;330:956-961.

104. Horwitz PA, Berlin JA, Sauer WH et al. Registry Committee of the Society for Cardiac Angiography Interventions. Bleeding risk of platelet glycoprotein IIb/IIla receptor antagonists in broad-based practice (results from the Society for Cardiac Angiography and Interventions Registry). Am J Cardiol $2003 ; 91: 803-806$.

105. Sadeghi HM, Grines CL, Chandra HR et al. Percutaneous coronary interventions in octogenarians. glycoprotein IIb/IIla receptor inhibitors' safety profile. J Am Coll Cardiol $2003 ; 42: 428-432$

106. Coukell AJ. Clopidogrel. Drugs 1997; 54: 745-750.

107. Dyke CM. Safety of glycoprotein IIb/IIla inhibitors- a heart surgeon's perspective. Am Heart J 1999; 138:307-316.

108. Shlansky-Goldberg R. Platelet aggregation inhibitors for use in peripheral vascular interventions: what can we learn from the experience in the coronary arteries? J Vasc Interv Radiol 2002;13:229-246.

109. Samama CM, Bastien O, Forestier F et al. French Society of Anesthesiology and Intensive Care. Antiplatelet agents in the perioperative period: expert recommendations of the French Society of Anesthesiology and Intensive Care (SFAR) 2001-- summary statement. Can J Anaesth 2002 ;49:S26-S35.

110. Haljamae H. Thromboprophylaxis, coagulation disorders, and regional anaesthesia. Acta Anaesthesiol Scand 1996;40: 1024-1040

111. Muir JJ, Church EJ, Weinmeister KP. Epidural hematoma associated with dextran infusion. South Med J 2003;96:811-814.

112. Blanloeil Y, Trossaert M, Rigal JC et al. Effects of plasma substitutes on hemostasis. Ann Fr Anesth Reanim 2002;21:648667.

113. Xi M, Beguin S. The relative importance of the factors II, VII, IX and X for the prothrombinase activity in plasma of orally anticoagulated patients. Thromb Haemost 1989;62:788-791.

114. Shields RC, McBane RD, Kuiper JD et al. Efficacy and safety of intravenous phytonadione (Vitamin $\mathrm{K}_{1}$ ) in patients on longterm oral anticoagulant therapy. Mayo Clin Proc 2001; 76: 260-266.

115. Wille-Jorgensen $P$, Jorgensen $L N$, Rasmussen LS. Lumbar regional anesthesia and prophylactic anticoagulant therapy: is the combination safe? Anaesthesia 1991; 46: 623-627.

116. Pullarkat VA, Kalapura T, Pincus $M$ et al Intraspinal hemorrhage complicating oral anticoagulant therapy: an unusual case of cervical hematomyelia and a review of the literature. Arch Intern Med 2000 24;160: 237-240.

117. van der Meer FJ, Rosendaal FR, Vandenbroucke JPet al. Assessment of a bleeding risk index in two cohorts of patients treated with oral anticoagulants. Thromb Haemost 1996;76:12-16.

118. Parziale JR, Marino AR, Herndon JH. Diagnostic peripheral nerve block resulting in compartment syndrome. Case report. Am J Phys Med Rehabil 1988;67:82-84.

119. Watts SA, Gibbs NM. Outpatient management of the chronically anticoagulated patient for elective surgery. Anaesth Intensive Care 2003;31:145-154.

120. Kearon C, Hirsh J Management of anticoag ulation before and after elective surgery. $N$ Engl J Med 1997;336:1506-1511.

121. Thumboo J, O'Duffy JD. A prospective study of the safety of joint and soft tissue aspirations and injections in patients taking warfarin sodium. Arthritis Rheum 1998;41:736-739.

122. Gerson LB, Gage BF, Owens DK, Triadafilopoulos G. Effect and outcomes of the ASGE guidelines on the periendoscopic management of patients who take anticoagulants. Am J Gastroenterol 2000 ;95: 1717-1724.

123. Katholi RE, Nolan SP, McGuire LB. The management of anticoagulation during noncardiac operations in patients with prosthetic heart valves: a prospective study. Am Heart J 1978;96:163-165.

124. Tinker JH, Tarhan S. Discontinuing anticoagulant therapy in surgical patients with heart valve prostheses: observations in 
180 operations. JAMA 1978;239:738-739.

125. Ananthasubramaniam K, Beattie JN, Rosman HS et al. How safely and for how long can warfarin therapy be withheld in prosthetic heart valve patients hospitalized with a major hemorrhage? Chest 2001; 119:478-484.

126. Maduro JA, Rookstool M, Wease G. The management of patients on chronic Coumadin therapy undergoing subsequent surgical procedures. Am Surg 1994;60: 542-547.

127. Caliendo FJ, Halpern VJ, Marini CP et al. Warfarin anticoagulation in the perioperative period: is it safe? Ann Vasc Surg 1999;13:11-16.

128. Gould MK, Dembitzer AD, Doyle RL, Hastie TJ, Garber AM. Low-molecular-weight heparins compared with unfractionated heparin for treatment of acute deep venous thrombosis: a meta-analysis of randomized, controlled trials. Ann Intern Med 1999;130:800-809.

129. Rosenquist RW, Brown DL. Neuraxial bleeding: fibrinolytics/thrombolytics. Reg Anesth Pain Med 1998;23:152-156.

130. Holst J, Lindblad B, Bergqvist D et al. The effect of protamine sulphate on plasma tissue factor pathway inhibitor released by intravenous and subcutaneous unfractionated and low molecular weight heparin in man. Thromb Res 1997;86:343-348.

131. Hirsh J, Raschke R, Warkentin TE et al. Mechanism of action, pharmacokinetics, dosing considerations, monitoring, efficacy, and safety. Chest 1995; 108:258S275 S.

132. Hirsh J, Warkentin TE, Shaughnessy SG et al. Heparin and low-molecular-weight heparin : Mechanisms of action, pharmacokinetics, dosing, monitoring, efficacy, and safety. Chest 2001; 119 : S64-S94.

133. Liu SS, Mulroy MF. Neuraxial anesthesia and analgesia in the presence of standard heparin. Reg Anesth Pain Med 1998 ;23: 157-163.

134. Osipova NA, Vetsheva MS, Reshetov IV et al. Special aspects of anesthesiology and intensive care in oncological operations with microsurgical autoplasty. Anesteziol Reanimatol 2001;5:10-16.

135. Collins R, Scrimgeour A, Yusuf S, Peto R. Reduction in fatal pulmonary embolism and venous thrombosis by perioperative administration of subcutaneous heparin. Overview of results of randomized trials in general, orthopedic, and urologic surgery. New Engl J Med 1988; 318:1162-1173.

136. Gallus AS, Hirsh J, Tuttle RJ et al. Small subcutaneous doses of heparin in prevention of venous thrombosis. NEJM 1973; 288:545-551.

137. Dehring DJ, Arens JF. Pulmonary thromboembolism: disease recognition and patient management. Anesthesiology 1990 ;73:146-164.

138. Levine M, Raskob G, Landefeld S et al. Hemorrhagic complications of anticoagulant therapy. Chest 2001; 119:108S-121S.
139. Holst J, Lindblad B, Bergqvist D et al. Protamine neutralization of intravenous and subcutaneous low-molecular-weight heparin (tinzaparin, Logiparin ${ }^{T M}$ ). An experimental investigation in healthy volunteers. Blood Coagul Fibrinolysis 1994;5: 795-803.

140. Levine MN, Planes A, Hirsh J. The relationship between anti-factor Xa level and clinical outcome in patients receiving enoxaparin low molecular weight heparin to prevent deep vein thrombosis after hip replacement. Thromb Haemost 1989;62: 940-944.

141. Bergqvist D, Lindblad B, Matzsch T. Risk of combining low molecular weight heparin for thromboprophylaxis and epidural or spinal anesthesia. Semin Thromb Hemost 1993;19:147-151

142. Bergqvist D, Lindblad B, Matzsch T. Low molecular weight heparin for thromboprophylaxis and epidural/spinal anaesthesia-Is there a risk? Acta Anaesthesiol Scand 1992;36:605-609.

143. Harenberg J, Gnasso A, De Vries JX et al. Inhibition of low molecular weight heparin by protamine chloride in vivo. Thromb Res $1985 ; 38: 11-20$.

144. Lojewski B, Bacher P, Iqbal O et al. Evaluation of hemostatic and fibrinolytic alterations associated with daily administration of low-molecular-weight heparin for a 12-week period. Semin Thromb Hemost 1995;21:228-239.

145. Klein SM, D’Ercole F, Greengrass RA et al. Enoxaparin associated with psoas hematoma and lumbar plexopathy after lumbar plexus block. Anesthesiology 1997 ;87: 1576-1579.

146. Turpie A, Levine M, Hirsh J et al. A randomized controlled trial of a low-molecular-weight heparin (enoxaparin) to prevent deep-vein thrombosis in patients undergoing elective hip surgery. NEJM 1986; 315:925-929.

147. Levine M, Hirsh J, Gent M et al. Prevention of deep vein thrombosis after elective hip surgery. A randomized trial comparing low molecular weight heparin with standard unfractionated heparin. Ann Intern Med 1991; 114:545-551.

148. Heit JA. Perioperative management of the chronically anticoagulated patient [Review]. J Thrombosis Thrombolysis 2001; 12:81-87.

149. Bergqvist D. Enoxaparin: a pharmacoeconomic review of its use in the prevention and treatment of venous thromboembolism and in acute coronary syndromes. Pharmacoeconomics 2002;20:225-243

150. Logason K, Bergqvist D; Study Group on Antothrombotic Prophylaxis of Femorodistal Bypass Surgery. Low molecular weight heparin (enoxaparin) versus dextran in the prevention of early occlusion following arterial bypass surgery distal to the groin. Eur J Vasc Endovasc Surg 2001;21:261265.

151. Apitz-Castro R, Escalante J, Vargas R et al.
The antiplatelet principle of garlic, synergistically potentiates the antiaggregatory action of prostacyclin, forskolin, indomethacin, dypiramidole on human platelets. Thrmob Res 1986; 42:303-311

152. Kaye AD, Heavner JE, Sabar R Nutraceuticals and risk of neuraxial bleeding. Reg Anesth Pain Med 2001;26:92-93.

153. Chung KF, Dent G, McCusker M et al. Effect of a ginkgolide mixture (BN 52063) in antagonising skin and platelet responses to platelet activating factor in man. Lancet $1987 ; 1: 248-851$.

154. Matthews MK Jr. Association of Ginkgo biloba with intracerebral hemorrhage. $\mathrm{Neu}$ rology 1998;50:1933-1934.

155. Rowin J, Lewis SL. Spontaneous bilateral subdural hematomas associated with chronic Ginkgo biloba ingestion.Neurology 1996;46:1775-1776.

156. Vale S. Subarachnoid haemorrhage associated with Ginkgo biloba. Lancet 1998 ;352:36.

157. Kuo SC, Teng CM, Lee JCet at. Antiplatelet components in Panax ginseng. Planta Med 1990 Apr;56:164-167.

158. Attele AS, Wu JA, Yuan CS.Ginseng pharmacology: multiple constituents and multiple actions. Biochem Pharmacol 1999 ;58:1685-1693.

159. Kaye AD, Clarke RC, Sabar R et al. Herbal medicines: current trends in anesthesiology practice--a hospital survey. J Clin Anesth. 2000 Sep;12:468-471.

16o. Direct Thrombin Inhibitor Trialists' Collaborative Group. Direct thrombin inhibitors in acute coronary syndromes: principal results of a meta-analysis based on individual patients' data. Lancet 2002;359:294302.

161. Eikelboom J, White H, Yusuf S. The evolving role of direct thrombin inhibitors in acute coronary syndromes. J Am Coll Cardiol. 2003 Feb 19;41:70S-78S

162. Argatroban prescribing information, Texas Biotechnology Corporation and GlaxoSmithKline.

163. Turpie AG, Eriksson BI, Lassen MR et al. Fondaparinux, the first selective factor $\mathrm{Xa}$ inhibitor. Curr Opin Hematol 2003 Sep;10: 327-332.

164. Landow L, Bedford RA. Low-molecular weight heparin, spinal hematomas, and the FDA: what's wrong with this picture? Reg Anesth Pain Med 1999;24:8-10.

165. Mannucci PM. Hemostatic drugs. N Engl J Med. 1998 23;339:245-253

166. Schmitz A, Wallny T, Sommer T et al. Spinal epidural haematoma in haemophilia A. Haemophilia. 1998;4:51-55.

167. Harvie A, Lowe GD, Forbes CD et al. Intraspinal bleeding in haemophilia: successful treatment with factor VIII concentrate. J Neurol Neurosurg Psychiatry 1977 ;40: 1220-1223.

168. Leach M, Makris M, Hampton KK et al. Spinal epidural haematoma in haemophilia A with inhibitors--efficacy of recombinant 
factor VIla concentrate. Haemophilia 1999 ;5:209-212.

169. Park P, Fewel ME, Thompson BG et al. Recombinant activated factor VII for the rapid correction of coagulopathy in nonhemophilic neurosurgical patients. Neurosurgery 2003;53:34-38; discussion 38-39.

170. Cohen S, Daitch JS, Amar D et al. Epidural analgesia for labor and delivery in a patient with von Willebrand's disease. Reg Anesth 1989;14:95-97.

171. Kang SB, Rumball KM, Ettinger RS. Continuous axillary brachial plexus analgesia in a patient with severe hemophilia. J Clin Anesth 2003; 15: 38-40.

172. Kakazu K, Ohira N, Ojima T, Oshida M, Akiyama M, Horaguchi M, Takahashi S. Extensive spinal epidural hemorrhage associated with von Willebrand's disease--a case report. Nippon Seikeigeka Gakkai Zasshi 1980;54:501-505.

173. Milaskiewicz RM, Holdcroft A, Letsky E. Epidural anaesthesia and Von Willebrand's disease. Anaesthesia 1990; 45: 462-464.

174. Yarnell RW, D’Alton ME. Epidural hematoma complicating cholestasis of pregnancy. Curr Opin Obstet Gynecol 1996;8:239242.

175. Rolbin SH, Abbott D, Musclow E et al. Epidural anesthesia in pregnant patients with low platelet counts. Obstet Gynecol 1988 ;71:918-920.

176. Waldman SD, Feldstein G. Caudal administration of morphine sulfate in anticoagulated and thrombocytopenic patients. Anesth Analg 1987;66:267-268.

177. Beilin Y, Bodian CA, Haddad EM et al. Practice patterns of anesthesiologists regarding situations in obstetric anesthesia where clinical management is controversial. Anesth Analg 1996;83:735-741.

178. Beilin Y. Safe epidural analgesia in thirty parturients with platelet counts between 69,000 and 98,000 mm(-3). Anesth Analg 1997; 85: 385-388.

179. Rasmus KT, Rottman RL, Kotelko DM et al. Unrecognized thrombocytopenia and regional anesthesia in parturients: a retrospective review. Obstet Gynecol 1989 ;73: 943-946.

180. Halpern S, Crosby E. Unrecognized thrombocytopenia and regional anesthesia in parturients: a retrospective review [Letter] Obstet Gynecol 1989;74:971-972.

181. Abramovitz S, Beilin Y. Thrombocytopenia, low molecular weight heparin, and obstetric anesthesia. Anesthesiol Clin North America. 2003;21:99-109.

182. Breuer AC, Tyler HR, Marzewski DJ et al. Radicular vessels are the most probable source of needle-induced blood in lumbar puncture: significance for the thrombocytopenic cancer patient. Cancer 1982 15;49:2168-2172.

183. Chang KH, Ogawa M, Uchida K et al. Spinal anesthesia in a parturient complicated with idiopathic thrombocytopenic purpura. Masui 2003;52:893-896.
184. Dunn D, Dhopesh V, Mobini J. Spinal subdural hematoma: a possible hazard of lumbar puncture in an alcoholic. JAMA 1979;241:1712-1713.

185. Harvey SC, Roland PJ, C uré JK et al. Spinal epidural hematoma detected by lumbar epidural puncture. Anesth Analg 1997 ;84: 1136-1139.

186. Inoue $\mathrm{K}$,Yokoyama $\mathrm{M}$, Nakatsuka $\mathrm{H}$ et al. Spontaneous Resolution of Epidural Hematoma after Continuous Epidural Analgesia in a Patient without Bleeding Tendency. Anesthesiology 2002; 97: 735-737.

187. Morisaki H, Doi J, Ochiai R et al. Epidural hematoma after epidural anesthesia in a patient with hepatic cirrhosis. Anesth Analg 1995;80: 1033-1035.

188. Laglia AG, Eisenberg RL, Weinstein PR et al. Spinal epidural hematoma after lumbar puncture in liver disease. Ann Intern Med 1978;88:515-516.

189. Grejda S, Ellis K, Arino P. Paraplegia following spinal anesthesia in a patient with chronic renal failure. Regional Anesthesia 1989; 14:155-157.

190. Takahashi K, Koiwa F, Tayama H et al. A case of acute spontaneous epidural haematoma in a chronic renal failure patient undergoing haemodialysis: successful outcome with surgical management. Nephrol Dial Transplant 1999;14:24992501.

191. Ziyal IM, Aydin S, Inci S, et al. Multilevel acute spinal epidural hematoma in a patient with chronic renal failure--case report. Neurol Med Chir (Tokyo) 2003 ;43: 409-412.

192. Basta M, Sloan P. Epidural hematoma following epidural catheter placement in a patient with chronic renal failure. Can J Anaesth. 1999;46:271-274.

193. Levine MN, Goldhaber SZ, Gore JM et al. Hemorrhagic complications of thrombolytic therapy in the treatment of myocardial infarction and venous thromboembolism. Chest 1995; 108:291S-301S.

194. Hittelet A, Deviere J. Management of anticoagulants before and after endoscopy. Can J Gastroenterol 2003;17:329-332.

195. Konstantatos A. Anticoagulation and cataract surgery: a review of the current literature. Anaesth Intensive Care 2001;29:1118.

196. Kallio H, Paloheimo M. Maunuksela EL Haemorrhage and risk factors associated with retrobulbar/peribulbar block: a prospective study in 1383 patients. $\mathrm{Br} / \mathrm{An}$ aesth. 2000;85:708-711.

197. McMahan LB. Anticoagulants and cataract surgery. J Cataract Refract Surg 1988 ;14: 569-671.

198. Hall DL, Steen WH Jr, Drummond JW et al. Anticoagulants and cataract surgery. Ophthalmic Surg 1988;19:221-222.

199. Owens EL, Kasten GW, Hessel EA 2nd. Spinal subarachnoid hematoma after lumbar puncture and heparinization: a case report, review of the literature, and discussion of anesthetic implications. Anesth
Analg 1986;65:1201-1207.

200. Lumpkin MM.FDA public health advisory. Anesthesiology. 1998;88:27A-28A.

201. Stafford-Smith M. Impaired haemostasis and regional anaesthesia. Can J Anaesth 1996;43:R129-R141.

202. Dahlgren N, Tornebrandt K. Neurological complications after anaesthesia. A followup of 18000 spinal and epidural anesthetics performed over 3 years. Acta Anaesthesiol Scand 1995; 39:872-880.

203. Horlocker TT, Wedel DJ. Anticoagulation and neuraxial block: historical perspective, anesthetic implications, and risk management. Reg Anesth Pain Med 1998; 23:129-134

204. Bergqvist D, Wu CL, Neal JM. Anticoagulation and neuraxial regional anesthesia: perspectives. Reg Anesth Pain Med 2003 ;28:163-166.

205. Wu CL. Regional anesthesia and anticoagulation. Journal of Clinical Anesthesia 2001;13:49-58.

206. Wulf H. Thromboembolieprophylaxe un ruckenmarksnahe regionalanasthesie. $\mathrm{An}$ asthesiol Intensivmed 1995; 36:216-217.

207. Wulf H. Epidural anaesthesia and spinal hematoma. Can J. Anaesthesia 1996; 43: 1260-1271.

208. Vandermeulen EP. Van Aken H. Vermylen J. Anticoagulants and spinal-epidural anesthesia. Anesthesia \& Analgesia 1994; 79: 1165-1177.

209. Urmey WF, Rowlingson JC: Do antiplatelet agents contribute to the development of perioperative spinal hematoma? Reg Anesth and Pain Med 1998; 23:146-151.

210. Horlocker TT, Wedel DJ. Neuraxial block and low molecular weight heparin: balancing perioperative analgesia and thromboprophylaxis. Reg Anesth Pain Med 1998; 23:164-177.

211. Wagner S, Forsting M , Hache W : Spontaneous resolution of a large spinal epidural hematoma : Case report . Neurosurgery $1996 ; 38: 816-818$

212. Heye N. Is there a link between acute spinal epidural hematoma and aspirin? Spine 1995;20:1931-1932.

213. Locke GE, Giorgio AJ, Biggers SL Jr et al. Acute spinal epidural hematoma secondary to aspirin-induced prolonged bleeding. Surg Neurol 1976;5:293-296.

214. Weber J, Hoch A, Kilisek Let al. Spontaneous intraspinal epidural hematoma secondary to use of platelet aggregation inhibitors. Dtsch Med Wochenschr 2001;126: 876-878.

215. Thwaites BK, Nigus DB, Bouska GW, Mongan PD, Ayala EF, Merrill GA. Intravenous ketorolac tromethamine worsens platelet function during knee arthroscopy under spinal anesthesia. Anesth Analg;82:11761181.

216. Gilbert A, Owens BD, Mulroy MF. Epidural hematoma after outpatient epidural anesthesia. Anesth Analg $2002 ; 94: 77-78$

217. Gerancher JC, Waterer R, Middleton J. Tran- 
sient paraparesis after postdural puncture spinal hematoma in a patient receiving ketorolac. Anesthesiology 1997;86:490-494.

218. Williams KN, Jackowski A, Evans PJ. Epidural haematoma requiring surgical decompression following repeated cervical epidural steroid injections for chronic pain. Pain 1990;42:197-199.

219. Zink M, Rath M, Waltensdorfer A et al. Unilateral presentation of a large epidural hematoma. Anesthesiology 2003;98:10321033.

220. MacDonald R. Aspirin and extradural blocks. British Journal of Anaesthesia 1991; 66:1-3.

221. CLASP (Collaborative Low-dose Aspirin Study in Pregnancy) Collaborative Group.CLASP: a randomised trial of lowdose aspirin for the prevention and treatment of pre-eclampsia among 9364 pregnant women. Lancet 1994 ;343:619-629.

222. Horlocker TT, Bajwa ZH, Ashraf Z et al. Risk assessment of hemorrhagic complications associated with nonsteroidal antiinflammatory medications in ambulatory pain clinic patients undergoing epidural steroid injection. Anesth Analg 2002;95: 1691-1697

223. Horlocker TT, Wedel DJ, Offord KP. Does pre-operative anti-platelet therapy increase the risk of hemorrhagic complications associated with regional anesthesia? Anesth Analg 1990;631-634.

224. Horlocker TT, Wedel DJ, Schroeder DRet al Preoperative antiplatelet therapy does not increase the risk of spinal hematoma associated with regional anesthesia. Anesth Analg 1995;80:303-309.

225. Bell, WR. Commentary on 'Horlocker TT, Wedel DJ, Schroeder DR et al. Preoperative antiplatelet therapy does not increase the risk of spinal hematoma associated with regional anesthesia. Anesth Analg 1995 ;80:303-309.

226. Edelson RN, Chernik NL, Posner JB. Spinal subdural hematomas complicating lumbar puncture. Archives of Neurology 1974. 31: 134-137.

227. Mayumi T, Dohi S, Takahashi T. Spinal subarachnoid hematoma after lumbar puncture in a patient receiving antiplatelet therapy. Anesth Analg. 1983;62:777-779.

228. Pryle BJ, Carter JA, Cadoux-HudsonDelayed paraplegia following spinal anaesthesia. Spinal subdural haematoma following dural puncture with a $25 \mathrm{G}$ pencil point needle at T12-L1 in a patient taking aspirin. Anaesthesia 1996;51:263-265.

229. Knowles PR, Randall NP, Lockhart AS. Vascular trauma associated with routine spinal anaesthesia. Anaesthesia 1999;54: 647-650.

230. Spansberg NL, Anker-Moller E, Dahl JB et al. The value of continuous blockade of the lumbar plexus as an adjunct to acetylsalicyclic acid for pain relief after surgery for femoral neck fractures. Eur J Anaesthesiol 1996;13:410-412.

231. Guise PA. Sub-Tenon anesthesia: a pro- spective study of 6,000 blocks. Anesthesiology 2003;98:964-968.

232. Anderson KK, Larson NH, Saga-Rumley SA, Hamed LM. Spontaneous orbital hemorrhage during general anesthesia and arthroplasty. J Clin Anesth 1994 ;6:145-147.

233. Maier C, Gleim M, Weiss T, Stachetzki U, Nicolas V, Zenz M. Severe bleeding following lumbar sympathetic blockade in two patients under medication with irreversibl platelet aggregation inhibitors. Anesthesiology 2002; 97:740-743.

234. Benzon HT, Wong HY, Siddiqui T et al. Caution in performing epidural injections in patients on several antiplatelet drugs. Anesthesiology 1999;91:1558-1559.

235. Kawaguchi S, Tokutomi S. A case of epidural hematoma associated with epidural catheterization which occurred on 12th days after the last medication of ticlopidine hydrochloride. Masui 2002;51:526528.

236. Kroenke K, Gooby-Toedt D, Jackson JL. Chronic medications in the perioperative period. South Med J 1998;91:358-364.

237. Odoom JA, Sih IL. Epidural analgesia and anticoagulant therapy. Experience with one thousand cases of continuous epidurals. Anaesthesia 1983;38:254-259.

238. Wu CL, Perkins FM. Oral anticoagulant prophylaxis and epidural catheter removal. Reg Anesth 1996;21:517-524.

239. Horlocker TT, Wedel DJ, Schlicting JL. Postoperative epidural analgesia and oral anticoagulant therapy. Anesth and Analg 1994; 79:89-93.

240. Woolson ST, Robinson RK, Khan NQ, Rogers BS, Maloney WJ. Deep venous thrombosis prophylaxis for knee replacement: warfarin and pneumatic compression. Am J Orthopedics 1998; 27: 299-304

241. Badenhorst $\mathrm{CH}$. Epidural hematoma after epidural pain control and concomitant post-operative anticoagulation. Regional Anesthesia and Pain Medicine 1996; 21: 272-273.

242. Harder S, Thurmann P. Clinically important drug interactions with anticoagulants: an update. Clin Pharmakokinetics 1996; 30: 416-444

243. Enneking FK, Benzon H. Oral anticoagulants and regional anesthesia: a perspective. Reg Anesth Pain Med 1998 ;23:140145.

244. Pellegrino SV, Berardi TR. Dental management of patients on anticoagulant therapy. Gen Dent 1995 ;43:351-354.

245. Cohen AF, Warman SP Upper airway obstruction secondary to warfarin-induced sublingual hematoma. Arch Otolaryngol Head Neck Surg 1989;115:718-720.

246. Connolly Jr ES, Winfree CJ, McCormick PC. Management of spinal epidural hematoma after tissure plasminogen activator: a case report. Spine 1996;21:1694-1698

247. Cohen JE, Ginsberg HJ, Emery D et al.Fatal spontaneous spinal epidural hematoma following thrombolysis for myocardial in- farction. Surg Neurol 1998;49:520-522; discussion 523.

248. Groen RJM, Ponssen H. The spontaneous spinal epidural hematoma : A study of the etiology. J Neurol Sci 1990;98:121-138.

249. Groen RJM, Groenewegen HJ, Van Alphen HAM, Hoogland PVJM. Morphology of the human internal vertebral venous plexus : A cadaver study after intravenous Araldite CY 221 injection. Anat Rec 1997;249:285294.

250. Groen RJM, Van Alphen HAM. Operative treatment of spontaneous spinal epidural hematomas : A study of the factors determining postoperative outcome. Neurosurgery 1996;39:484-509.

251. Dickman CA, Shedd SA, Spetzler RF et al. Spinal epidural hematoma associated with epidural anesthesia: complications of systemic heparinization in patients receiving peripheral vascular thrombolytic therapy. Anesthesiology 1990;72:947950.

252. Onischuk JL, Carlsson C. Epidural hematoma associated with epidural anesthesia: complications of anticoagulant therapy. Anesthesiology 1992; 77:1221-1223.

253. Rabito SF, Ahmed S, Feinstein L et al. Intrathecal bleeding after intra-operative use of heparin and urokinase during continuous spinal anesthesia. Anesth and Analg 1996; 82:49-411.

254. Tryba M. European practice guidelines: thromboembolism prophylaxis and regional anesthesia. Reg Anesth Pain Med 1998; 23;178-182.

255. Tryba M, Wedel DJ. Central neuraxial block and low molecular weight (enoxaparin): lessons learned from different dosage regimes in two continents. Acta Anaesthesiol Scand Suppl 1997; 111: 100-104.

256. Stanton-Hicks MD. Low-dose heparin therapy and spinal anesthesia. JAMA 1981; 246:886

257. Darnat S, Guggiari M, Grob R et al. A case of spinal extradural hematoma during the insertion of an epidural catheter. $\mathrm{Ann} \mathrm{Fr}$ Anesth Reanim 1986;5:550-552.

258. Metzger G, Singbartl G. Spinal epidural hematoma following epidural anesthesia versus spontaneous spinal subdural hematoma. Two case reports. Acta Anaesthesiol Scand 1991;35:105-107.

259. Millar FA, Mackenzie A, Hutchison G et al. Hemostasis-altering drugs and central neural block. A survey of anesthetic practice in Scotland and the United Kingdom. Regional Anesthesia 1996; 21:529-533.

260. Sandhu H, Morley-Forster P, Spadafora S. Epidural hematoma following epidural analgesia in a patient receiving unfractionated heparin for thromboprophylaxis. Reg Anesth and Pain 2000; 25:72-75.

261. Wildsmith JA, McClure JH. Anticoagulant drugs and central nerve blockade. Anaesthesia 1991;46:613-614.

262. Greaves JD. Serious spinal cord injury due to haematomyelia caused by spinal anaesthesia in a patient treated with low-dose 
heparin. Anaesthesia 1997; 52:150-154.

263. Rao TL, El-Etr AA. Anticoagulation following placement of epidural and subarachnoid catheters: an evaluation of neurologic sequelae. Anesthesiology $1981 ; 55$ :618620.

264. Ruff RL, Dougherty JH Jr. Complications of lumbar puncture followed by anticoagulation. Stroke 1981;12:879-881.

265. Baron HC, LaRaja RD, Rossi G et al. Continuous epidural analgesia in the heparinized vascular surgical patient: a retrospective review of 912 patients. I Vasc Surg 1987; 6:144-146.

266. Canto M, Casas A, Sanchez MJ et al. Thoracic epidurals in heart valve surgery: neurologic risk evaluation. J Cardiothorac Vasc Anesth 2002;16:723-726.

267. Sanchez R, Nygard E. Epidural anesthesia in cardiac surgery: is there an increased risk? Cardiothorac Vasc Anesth 1998 ;12: 170-173.

268. Ho AM, Chung DC, Joynt GM. Neuraxial blockade and hematoma in cardiac surgery: estimating the risk of a rare adverse event that has not (yet) occurred.Chest 2000;117:551-555.

269. Chaney MA. Intrathecal and epidural anesthesia and analgesia for cardiac surgery. Anesth Analg 1997;84:1211-1221.

270. Baxter AD, Flynn JF, Jennings FO. Continuous intercostal nerve blockade. $\mathrm{Br} / \mathrm{An}$ aesth 1984:56:665-666.

271. Nielsen $\mathrm{CH}$. Bleeding after intercostal nerve block in a patient anticoagulated with heparin. Anesthesiology $1989 ; 71$ : 162-164.

272. Mani M, Ramamurthy N, Rao TL et al. An unusual complication of brachial plexus block and heparin therapy. Anesthesiology. 1978;48:213-214.

273. Schwander D, Bachmann F. Heparin and spinal or epidural anesthesia: decision analysis. Ann Fr Anesth Reanim 1991;10: 284-296.

274. Douketis JD, Kinnon K, Crowther MA. Anticoagulant effect at the time of epidural catheter removal in patients receiving twice-daily or once-daily low molecularweight heparin and continuous epidural analgesia after orthopedic surgery

275. Herbstreit F. Conservative treatment of paraplegia after removal of an epidural catheter during low-molecular-weight heparin treatment. Anesthesiology 2002; 97: 733-734.

276. Schroeder DR. Statistics: Detecting a rare adverse drug reaction using spontaneous reports. Reg Anesth and Pain Med 1998; 23:183-189.

277. Zufferey P, Laporte S, Quenet S et al. Optimal low-molecular-weight heparin regimen in major orthopaedic surgery. A metaanalysis of randomized trials. Thromb Haemost 2003;90:654-661.

278. Melde SL. Enoxaparin-induced retroperitoneal hematoma. Ann Pharmacother 2003;37:822-824.
279. McEvoy MD. Noncompliance in the inpatient administration of enoxaparin in conjunction with epidural or spinal anesthesia. J Arthroplasty 2000; 15: 604-607.

280. Osmani O, Afeiche N, Lakkis S. Paraplegia after epidural anesthesia in a patient with peripheral vascular disease: case report and review of the literature with a description of an original technique for hematoma evacuation. Journal of Spinal Disorders 2000;13:85-87.

281. Litz RJ, Hubler M, Koch T, Albrecht DM. Spinal-epidural hematoma following epidural anesthesia in the presence of antiplatelet and heparin therapy. Anesthesiology 2001 ;95:1031-1033.

282. Korttila K, Lauritsalo K, Sarmo A et al. Suitability of plasma expanders in patients receiving low-dose heparin for prevention of venous thrombosis after surgery. Acta Anaesthesiol Scand. 1983;27:104-107.

283. Singelyn FJ, Vanderelst PE, Gouverneur JM. Extended Femoral Nerve Sheath Block After Total Hip Arthroplasty: Continuous Versus Patient-Controlled Techniques. Anesth Analg. 2001;92:455-459.

284. Rose KD, Croissant PD, Parliament CF, Levin MB. Spontaneous epidural hematoma with associated platelet dysfunction from excessive garlic ingestion: a case report. Neurosurgery 1990; 26: 880-882.

285. Sabar R, Kaye AD, Frost EA Perioperative considerations for the patient on herbal medicines.Middle East J Anesthesiol. 2001 Oct;16(3):287-314

286. Cannavo D.Use of neuraxial anesthesia with selective factor Xa inhibitors. Am J Orthop 2002;31:21-23.

287. Fransson Sg, Nylander E. Vascular injury following cardiac catheterization, coronary angiography, and coronary angioplasty. Eur Heart J 1994; 15:232-235.

288. Horlocker TT, Kufner RP, Bishop AT et al. The risk of persistent paresthesia is not increased with repeated axillary block. Anesth Analg 1999;88:382-387.

289. Horlocker TT, O’Driscoll SW, Dinapoli RP. Recurring brachial plexus neuropathy in a diabetic patient after shoulder surgery and continuous interscalene block. Anesth Analg 2000; 91:688-690.

290. Urban MK, Urquhart B. Evaluation of brachial plexus anesthesia for upper extremity surgery. Reg Anesth 1994; 19:175-182.

291. Selander D, Dhuner KG, Lundborg G. Peripheral nerve injury due to injection needles used for regional anesthesia. An experimental study of the acute effects of needle point trauma. Acta Anaesthesiol Scand 1977;21:182-188.

292. Selander D, Edshage S, Wolff T. Paresthesiae or no paresthesiae? Nerve lesions after axillary blocks. Acta Anaesthesiol Scand 1979;23:27-33.

293. Weber SC, Jain R. Scalene regional anesthesia for shoulder surgery in a community setting: an assessment of risk. J Bone Joint Surg Am. 2002;84:775-779.

294. Edge KR, Nicoll JM. Retrobulbar hemor- rhage after 12,500 retrobulbar blocks. Anesth Analg 1993;76:1019-1022.

295. Carroll RP. Blindness following lacrimal nerve block. Ophthalmic Surg 1982;13: 812-814.

296. Cyriac IC, Pineda R 2nd. Postoperative complications of periocular anesthesia. Int Ophthalmol Clin 2000;40:85-91.

297. Rubin AP. Complications of local anaes thesia for opthalmic surgery. Br J Anaesth 1995; 75:93-96.

298. Gunning KE, Collett BJ. Spontaneous retrobulbar haemorrhage following anaesthesia. Anaesthesia 1987;42:875-876.

299. Holt EP Jr. Fallacy Of Cervical Discography. Report Of 50 Cases In Normal Subjects. JAMA 1964 ;188:799-801.

300. Guyer RD, Ohnmeiss DD, Mason SL et al. Complications of cervical discography: findings in a large series. I Spinal Disord 1997;10:95-101.

301. Mishio M, Matsumoto T, Okuda Y, Kitajima T. Delayed severe airway obstruction due to hematoma following stellate ganglion block. Reg Anesth Pain Med 1998 ;23:516519.

302. Wulf H, Maier C. Complications and side ef fects of stellate ganglion blockade. Results of a questionnaire survey.Anaesthesist 1992;41:146-151.

303. Mahli A, Coskun D, Akcali DT. Aetiology of convulsions due to stellate ganglion block: a review and report of two cases. Eur J Anaesthesiol. 2002 ;19:376-380.

304. Korevaar WC, Burney RG, Moore PA. Convulsions during stellate ganglion block: a case report. Anesth Analg 1979 ;58:329330.

305. Dukes RR, Alexander LA. Transient lockedin syndrome after vascular injection during stellate ganglion block. Reg Anesth 1993;18:378-380.

306. Zeidman SM, Thompson K, Ducker TB. Complications of cervical discography: analysis of 4400 diagnostic disc injections. Neurosurgery 1995;37:414-417.

307. Connor PM, Darden BV. Cervical discography complications and clinical efficacy. Spine 1993;18:2035-2038.

308. Skirving DJ, Dan NG. A 20-year review of percutaneous balloon compression of the trigeminal ganglion. J Neurosurg 2001;94: 913-917.

309. Taha JM, Tew JM Jr. Comparison of surgical treatments for trigeminal neuralgia: reevaluation of radiofrequency rhizotomy. Neurosurgery. 1996;38:865-871

310. Sweet WH. Treatment of trigeminal neuralgia by percutaneous rhizotomy. In You mans J (ed). Neurological Surgery. W.B. Saunders Co, Philadelphia, 1990, pp 38883921.

311. Sanders M, Zuurmond WW. Efficacy of sphenopalatine ganglion blockade in 66 patients suffering from cluster headache: a 12- to 70-month follow-up evaluation. Neurosurg. 1997;87:876-880.

312. Stan TC, Krantz MA, Solomon DL et al. The 
incidence of neurovascular complications following axillary brachial plexus block using a transarterial approach. A prospective study of 1,000 consecutive patients. Reg Anesth 1995;20:486-492.

313. Pearce H, Lindsay D, Leslie K. Axillary brachial plexus block in two hundred consecutive patients. Anaesth Intensive Care 1996;24:453-458.

314. Borgeat A, Ekatodramis G, Kalberer F et al. Acute and nonacute complications associated with interscalene block and shoulder surgery: a prospective study. Anesthesiology 2001 Oct;95:875-880.

315. Borgeat A, Ekatodramis G, Dumont C. An evaluation of the infraclavicular block via a modified approach of the Raj technique. Anesth Analg. 2001;93:436-441.

316. Ben-David B, Stahl S. Axillary block complicated by hematoma and radial nerve injury. Reg Anest Pain Med 199; 24:264 266.

317. Cockings EE. Axillary block complicated by hematoma and radial nerve injury. Regional Anesth Pain Med 2000; 25:103.

318. Borgeat A, Dullenkopf A, Ekatodramis G et al. Evaluation of the lateral modified approach for continuous interscalene block after shoulder surgery. Anesthesiology 2003;99:436-442.

319. Chambers WA. Peripheral nerve damage and regional anaesthesia. $\mathrm{Br} /$ Anaesth 1992;69:429-430.

320. Naja Z, Lonnqvist PA. Somatic paravertebral nerve blockade. Incidence of failed block and complications. Anaesthesia 2001;56:1184-1188.

321. Dangoisse M, Collins S, Glynn CJ. Haemothorax after attempted intercostal catheterization. Anaesthesia 1994;49:961-963.

322. Thomas PW, Sanders DJ, Berrisford RG. $B r$ J Anaesth 1999;83:668-669.

323. Thomas PW, Sanders D, Sweeting CJ et al. Pulmonary haemorrhage after percutaneous paravertebral blockln defence of paravertebral blockade Br J Anaesth. 2002 ;88: 743; author reply 744 .

324. lida Y, Kashimoto S, Matsukawa T et al. A hemothorax after thoracic epidural anesthesia. J Clin Anesth 1994;6:505-507.

325. Schellhas KP, Pollei SR, Dorwart RH. Thoracic discography. A safe and reliable technique. Spine 1994;19:2103-2109.

326. Berger GS, Tyler CW, Harrod EK. Maternal deaths associated with paracervical block anesthesia. Am J of Obstetrics and Gynecology 1974; 118:1142

327. Gaylord T, Pearson J. Neuropathy following paracervical block in the obstetric patient. Obstetrics and Gynecology 1982;60:521524 .

328. Kurzel RB, Au AH, Rooholamini SA. Retroperitoneal hematoma as a complication of pudendal block. Diagnosis made by computed tomography. West J Med 1996 ;164: 523-525.

329. Vaisman J. Pelvic hematoma after an ilioinguinal nerve block for orchialgia. Anesth
Analg 2001;92:1048-1049.

330. Ghia JN, Blank JW, McAdams CG. A new interabdominis approach to inguinal region block for the management of chronic pain. Reg Anesth 1991;16:72-78.

331. Johr M. A complication of continuous blockade of the femoral nerve. Reg Anaesth 1987;10:37-38.

332. Zelman S. Fatal hemorrhage following needle biopsy. JAMA 1954; 154: 997-1000.

333. Ross JH, Ross IP. The value of renal biopsy. Lancet 1957; 2: 559-565.

334. Aida S, Takahashi H, Shimoji K. Renal subcapsular hematoma after lumbar plexus block. Anesthesiology 1996;84:452-455.

335. Brown E, Kunjappan V. Single needle lateral approach for lumbar sympathetic block. Anesth Analg 1975; 54:725-729.

336. Wheatley J, Motamedi F, Hammonds W. Page kidney resulting from a massive subcapsular hematoma: complication of lumbar sympathetic nerve block. Urology $1984 ; 24: 361-363$.

337. Guyer RD, Ohnmeiss DD, Vaccaro A. Lumbar discography. Spine J 2003 ;3:11-27.

338. di Benedetto P, Casati A, Bertini L et al. Postoperative analgesia with continuous sciatic nerve block after foot surgery: a prospective, randomized comparison between the popliteal and subgluteal approaches. Anesth Analg 2002;94:9961000.

339. White PF, Issioui T, Skrivanek GD et al. The use of a continuous popliteal sciatic nerve block after surgery involving the foot and ankle: does it improve the quality of recovery? Anesth Analg 2003;97:1303-1309.

340. Ilfeld BM, Morey TE, Wang RD et al. Continuous popliteal sciatic nerve block for postoperative pain control at home: a randomized, double-blinded, placebo-controlled study. Anesthesiology 2002;97:959-965.

341. Liu SS, Salinas FV. Continuous plexus and peripheral nerve blocks for postoperative analgesia. Anesth Analg 2003;96:263272.

342. Capdevila X, Biboulet P, Morau D et al. Continuous three-in-one block for postoperative pain after lower limb orthopedic surgery: where do the catheters go? Anesth Analg 2002;94:1001-1006.

343. Schon LC, Lam PW, Easley ME et al. Complex salvage procedures for severe lower extremity nerve pain. Clin Orthop 2001 Oct;391:171-180.

344. Gold MS. The effect of the epidural/ general and cervical plexus block on activated clotting time in patients undergoing vascular surgery. Anesth Analg 1993; 74: 701-704.

345. Salvati EA, Pellegrini VD Jr, Sharrock NE et al. Recent advances in venous thromboembolic prophylaxis during and after total hip replacement. J Bone Joint Surg Am 2000;82:252-270.

346. Bottner F, Sculco TP. Nonpharmacologic thromboembolic prophylaxis in total knee arthroplasty. Clin Orthop. 2001;392:249-
256.

347. Modig J, Borg T, Bagge L et al. Role of extradural and of general anaesthesia in fibrinolysis and coagulation after total hip replacement. Br J Anaesth 1983 ;55:625629.

348. Modig J, Borg T, Karlstrom G et al. Thromboembolism after total hip replacement: role of epidural and general anesthesia. Anesth Analg 1983;62:174-180.

349. Lindgren L, Silvanto M, Scheinin B et al. Erythrocyte counts in the cerebrospinal fluid associated with continuous spinal anaesthesia. Acta Anaesthesiol Scand 1995;39:396-400.

350. McNeill MJ, Thorburn J. Cannulation of the epidural space. A comparison of $18-$ and 16-gauge needles. Anaesthesia 1988 ;43: 154-155.

351. Alexiadou-Rudolf C, Ernestus R-I, Nanassis $\mathrm{K}$ et al. Acute nontraumatic spinal epidural hematomas : An important differential diagnosis in spinal emergencies. Spine 1998;23:1810-1813.

352. Nakamura H, Tominaga $\mathrm{T}$, Satoh $\mathrm{S}$ et al. Spontaneous spinal epidural hematoma: case report No Shinkei Geka 1997;25:379383.

353. Wittebol MC, van Veelen CW. Spontaneous spinal spidural haematoma. Etiological considerations. Clin Neurol Neurosurg 1984; 86: 265-270.

354. Abram SE, O’Connor TC. Complications associated with epidural steroid injections. Reg Anesth 1996;21:149-162.

355. Gutterman P. Acute spinal subdural hematoma following lumbar puncture. Surgical Neurology 1977. 7:355-356.

356. Ghaly RF Recovery after high-dose methylprednisolone and delayed evacuation: a case of spinal epidural hematoma. I Neurosurg Anesthesiol 2001;13:323-328.

357. Muranjan MN, Deshmukh CT. Acute transverse myelitis due to spinal epidural hematoma-first manifestation of severe hemophilia. Indian Pediatr 1999; 36:11511153.

358. Stoll A, Sanchez M. Epidural hematoma after epidural block: implications for its use in pain management. Surg Neurol 2002;57:235-240.

359. Vaya A, Resureccion M, Ricart JM et al. Spontaneous cervical epidural hematoma associated with oral anticoagulant therapy. Clin Appl Thromb Hemost 2001 ;7:166168.

36o. Ng WH, Lim CC, Ng PY et al. Spinal epidural haematoma: MRI-aided diagnosis. J Clin Neurosci 2002 ;9:92-94.

361. Zakowski M Complications associated with regional anesthesia in the obstetric patient. Semin Perinatol 2002;26:154168.

362. Dahlin PA, George J. Intraspinal hematoma as a complication of anticoagulant therapy. Clin Pharm 1984; 3:656-661.

363. Kreppel D, Antoniadis G, Seeling W. Spinal hematoma: a literature survey with meta- 
analysis of 613 patients. Neurosurgical Review 2003; 26: 1-49.

364. Lawton MT, Porter RW, Heiserman JE et al. Surgical management of spinal epidural hematoma : Relationship between surgical timing and neurological outcome. $J$ Neurosurg 1995; 83: 1-7.

365. Calliauw L, Dhara M, Martens F et al. Spinal epidural hematoma without lesion of the spine. Report of 4 cases. Clin Neurol Neurosurg 1988;90:131-136.

366. Foo D, Rossier AB. Preoperative neurological status in predicting surgical outcome of spinal epidural hematomas. Surg Neurol 1981;15:389-401.

367. Shioya H, Kikuchi K, Suda Y et al. Spontaneous spinal epidural hematoma with spontaneous remission, diagnosed in MRI on superacute stage. No To Shinkei 1998 ;50:447-452.

368. Futawatari K, Matsuoka S, Kowada M. A case of spinal epidural hematoma associated with idiopathic thrombocytopenic purpura. No Shinkei Geka 1991;19:11871190.

369. Muthukumar N. Chronic spontaneous spinal epidural hematoma -- a rare cause of cervical myelopathy. Eur Spine / 2003 ;12: 100-103.

370. Reitman CA, Watters W 3rd. Subdural hematoma after cervical epidural steroid injection. Spine 2002;27:E174-E176.

371. Yagi S, Hida K, Iwasaki Y et al. Cervical epidural hematoma caused by cervical twisting after epidural anesthesia: a case report. No Shinkei Geka 1998;26:627-632.

372. Botwin KP, Castellanos R, Rao S et al. Complications of fluoroscopically guided interlaminar cervical epidural injections. Arch Phys Med Rehabil. 2003;84:627-633.

373. Waldman SD. Complications of cervical epidural nerve blocks with steroids: a prospective study of 790 consecutive blocks. Reg Anesth 1989;14:149-151.

374. Cicala RS, Westbrook L, Angel JJ. Side effects and complications of cervical epidural steroid injections. J Pain Symptom Manage. 1989;4:64-66.

375. Rogers LA. Acute subdural hematoma and death following lateral cervical spinal puncture. J Neurosurg 1983 ;58:284-286.

376. Furman MB, Giovanniello MT, O‘Brien EM. Incidence of intravascular penetration in transforaminal cervical epidural steroid injections. Spine 2003;28:21-25

377. Tryba M. Epidural regional anesthesia and low molecular heparin: Pro. Anasthesiol Intensivmed Notfallmed Schmerzther 1993;28:179-181.

378. Vaes L. Regional Anesthesia:How Safe?. A review of 20,590 cases. Acta Anaesthesiologic Belgica 1988;39:175-176.

379. Moore DC, Bridenbaugh LD. Spinal (subarachnoid) block: a review of 11,574 cases JAMA 1966;195:907-912.

380. Bergmann H. Spinal anesthesia. Langenbecks Arch Chir 1977; 345:515-518.

381. Phillips OC, Ebner H, Nelson AT et al. Neu- rologic complications following spinal anesthesia with lidocaine: a prospective review of 10,440 cases. Anesthesiology 1969;55: 618-620.

382. Sadove MS, Levin MJ, Rant-Sejdinaj I. Neurological complications of spinal anaesthesia. Can Anaesth Soc / 1961;8:405-416.

383. Vandam LD, Dripps RD. Long-term followup of patients who received 10,098 spinal anesthetics. JAMA 1960; 172: 1483-1487.

384. Palot M, Visseaux H, Botmans $C$ et al. Epidemiology of complications of obstetrical epidural analgesia. Cah Anesthesiol. 1994;42:229-233.

385. Puke M, Norlander O. Sevre neurological complications in extradural and intrathecal blockades as reported to the Swedish Patient Insurance Consortium between 1980-1984. Schmerz/Pain/Douleur 1988; 9: 76-78.

386. Scott DB, Hibbard BM. Serious non-fatal complications associated with extradural block in obstetric practice. Br J Anaesth 1990;64:537-541.

387. Wulf $\mathrm{H}$, Striepling E. Postmortem findings after epidural anaesthesia. Anaesthesia. 1990;45:357-361.

388. Usubiaga JE. Neurological complications following epidural anesthesia. Int Anesthesiol Clin 1975;13:1-153.

389. Blomberg RG, Olsson SS. The lumbar epidural space in patients examined with epiduroscopy. Anesth Analg. 1989;68:157160.

390. Beck H, Brassow F, Doehn M et al. Epidural catheters of the multi-orifice type: dangers and complications. Acta Anaesthesiol Scand. 1986;30:549-555.

391. Verniquet AJ. Vessel puncture with epidural catheters. Experience in obstetric patients. Anaesthesia 1980;35:660-662.

392. Sullivan WJ, Willick SE, Chira-Adisai W et al. Incidence of intravascular uptake in lumbar spinal injection procedures. Spine 2000;25:481-486.

393. Renfrew DL, Moore TE, Kathol MH et al. Correct placement of epidural steroid injections: fluoroscopic guidance and contrast administration. AJNR Am J Neuroradiol 1991;12:1003-1007.

394. Furman MB, O‘Brien EM, Zgleszewski TM. Incidence of intravascular penetration in transforaminal lumbosacral epidural steroid injections. Spine 2000 15;25:26282632.

395. Botwin KP, Gruber RD, Bouchlas CG et al. Complications of fluoroscopically guided transforaminal lumbar epidural injections. Arch Phys Med Rehabil 2000;81:10451050.

396. Botwin KP, Gruber RD, Bouchlas CG et al. Complications of fluoroscopically guided caudal epidural injections. Am J Phys Med Rehabil 2001;80:416-424.

397. Vakharia SB, Thomas PS, Rosenbaum AE et al. Magnetic resonance imaging of cerebrospinal fluid leak and tamponade effect of blood patch in postdural puncture headache. Anesth Analg 1997;84:585590.

398. Beards SC, Jackson A, Griffiths AG, Horsman EL. Magnetic resonance imaging of extradural blood patches: appearances from 30 minutes to 18 hours. Br J Anaesth 1993; 71: 182-188.

399. Tekkok IH, Carter DA, Brinker R. Spinal subdural haematoma as a complication of immediate epidural blood patch. Can I Anaesth 1996;43:306-309.

400. McLain RF, Fry M, Hecht ST. Transient paralysis associated with epidural steroid injection. J Spinal Disord 1997;10:441-444.

401. Grillo PJ, Yu HC, Patterson RH Jr. Delayed intraspinal hemorrhage after dorsal column stimulation for pain. Arch Neurol 1974:30:105-106

402. Lao TT, Halpern SH, MacDonald D et al. Spinal subdural haematoma in a parturient after attempted epidural anaesthesia. Can J Anaesth 1993;40:340-345.

403. Greensite FS, Katz J. Spinal subdural hematoma associated with attempted epidural anesthesia and subsequent continuous spinal anesthesia. Anesth Analg 1980 ;59:72-73.

404. Kirkpatrick D, Goodman SJ. Combined subarachnoid and subdural spinal hematoma following spinal puncture. Surg Neurol 1975;3:109-111.

405. Shah RV, Heavner JE. Recognition of the subarachnoid and subdural compartments during epiduroscopy: two cases. Pain Practice 2003; 3: 321.

406. Eastwood DW. Hematoma after epidura anesthesia:relationship of skin and spinal angiomas. Anesth Analg 1991; 73:352354.

407. Ekatodramis G, Macaire P, Borgeat A. Prolonged Horner syndrome due to neck hematoma after continuous interscalene block. Anesthesiology 2001;95:801-803.

408. Sukhani R, Barclay J, Aasen M. Prolonged Horner's syndrome after interscalene block: a management dilemma. Anesth Analg 1994;79:601-603.

409. Forrest JB. An unusual complication after stellate ganglion block by the paratracheal approach: a case report. Can Anaesth Soc) 1976;23:435-439.

410. Bruyns $\mathrm{T}$, Devulder J, Vermeulen $\mathrm{H}$ et al. Possible inadvertent subdural block following attempted stellate ganglion blockade. Anaesthesia 1991;46:747-749.

411. Whitehurst L, Harrelson JM. Brain-stem anesthesia. An unusual complication of stellate ganglion block. J Bone Joint Surg Am 1977;59:541-542.

412. Stannard CF, Glynn CJ, Smith SP. Dural puncture during attempted stellate ganglion block. Anaesthesia 1990;45:952 954.

413. Van de Kelft E, De la Porte C, Meese G et al. Intracranial subdural hematoma after spinal anesthesia. Acta Anaesthesiologica Belgica 1991; 42:177-180.

414. Baldwin LN, Galizia EJ. Bilateral subdural 
haematomas: a rare diagnostic dilemma following spinal anaesthesia. Anaesth Intensive Care. 1993;21:120-121.

415. Bjarnhall M, Ekseth $\mathrm{K}$, Bostrom $\mathrm{S}$ et al. Intracranial subdural haematoma--a rare complication following spinal anaesthesia. Acta Anaesthesiol Scand 1996;40: 1249-1251.

416. Campbell DA, Varma TR. Chronic subdural haematoma following epidural anaesthesia, presenting as puerperal psychosis. $\mathrm{Br}$ J Obstet Gynaecol 1993;100:782-784.

417. Cohen JE, Godes J, Morales B. Postpartum bilateral subdural hematomas following spinal anesthesia: case report. Surg Neurol 1997;47:6-8.

418. Rudehill A, Gordon E, Rahn T. Subdural haematoma: A rare but life-threatening complication after spinal anaesthesia. Acta Anaesthesiol Scand 1983 27:376377.

419. Ortiz M. Aliaga L. Baturell C et al. Intracranial subdural haematoma--a rare complication after spinal anaesthesia. European Journal of Anaesthesiology 1991; 8:245-248.

420. Depreitere B, Van Calenbergh F, van Loon J. A clinical comparison of non-traumatic acute subdural hematomas either related to coagulopathy or of arterial origin without coagulopathy. Acta Neurochir (Wien) 2003; 145:541-546.

421. Morgan CM, Schatz H, Vine AK et al. Ocular complications associated with retrobulbar injections. Ophthalmology 1988 ;95:660-665.

422. Heavner J, Racz GB, Jenigiri B et al. Sharp Versus Blunt Needle: A Comparative Study of Penetration of Internal Structures and Bleeding in Dogs. Pain Practice 2003;3: 226-231.

423. Nelson JW. "Paraplegia after lumbosacral nerve root block. report of three cases", by Houten JK et al. Spine J 2003;3:88-89.

424. Chelly JE. How can we possibly prevent complications related to peripheral nerve blocks? Anesth Analg 2001;93:1080-1081.

425. Rice AS, McMahon SB. Peripheral nerve injury caused by injection needles used in regional anaesthesia: influence of bevel configuration, studied in a rat model. $\mathrm{Br}$ J Anaesth 1992;69:433-438.

426. Persson J, Flisberg P, Lundberg J. Thoracic epidural anesthesia and epidural hematoma: case report. Acta Anaesthesiol Scand 2002; 46:1171-1174.

427. Fredman B, Nun MB, Zohar E et al. Epidural steroids for treating "failed back surgery syndrome": is fluoroscopy really necessary? Anesth Analg 1999;88:367-372.

428. Shah RV, Heavner JE. Recognition of the subarachnoid and subdural compartments during epiduroscopy: two cases. Pain Practice 2003; 3: 321-325.

429. Baker R, Dreyfuss P, Mercer $S$ et al. Cervical transforaminal injection of corticosteroids into a radicular artery: a possible mechanism for spinal cord injury. Pain 2003;103:211-215.

430. Kopacz DJ, Allen HW. Comparison of needle deviation during regional anesthetic techniques in a laboratory model. Anesth Analg 1995;81:630-633.

431. Baumgarten RK. Importance of the nee- dle bevel during spinal and epidural. Reg Anesth 1995;20:234-238.

432. Drummond GB, Scott DH. Deflection of spinal needles by the bevel. Anaesthesia 1980;35:854-857.

433. Hill RP, Greengrass R. Pulmonary haemorrhage after percutaneous paravertebral block. BrJ Anaesth 2000;84:423-424.

434. Helperin SW, Cohen DD. Hematoma following epidural anesthesia: report of a case. Anesthesiology 1971; 35:642-644.

435. Janis KM. Epidural hematoma following post-operative epidural analgesia: a case report. Anesth Analg 1972: 51: 689-692.

436. Traeger KA. Hematoma following inferior alveolar injection: a possible cause for anesthesia failure. Anesth Prog 1979 ;26: 122-123.

437. Beyth RJ, Quinn LM, Landefeld CS. Prospective evaluation of an index for predicting the risk of major bleeding in outpatients treated with warfarin. Am J Med 1998;105:91-99.

438. Wells PS, Forgie MA, Simms $M$ et al. The outpatient bleeding risk index: validation of a tool for predicting bleeding rates in patients treated for deep venous thrombosis and pulmonary embolism. Arch Intern Med. 2003;163:917-920.

439. Barber A, Green D, Gallusso T et al. The bleeding time as a pre-operative screening test. Am J Med 1985;78:716-724.

440. Conolly M, Raj PP, Kunkel L. Management of a hemophiliac patient during an epidural steroid injection: a clinical case conference. Pain Digest 1995; 20-24. 
